\title{
A New Set of Isoreticular, Homochiral Metal-Organic Frameworks with ucp Topology
}

\author{
Michael Sartor, Timo Stein, Frank Hoffmann, and Michael Fröba*
}

Institute of Inorganic and Applied Chemistry, Department of Chemistry, University of Hamburg, Martin-Luther-King-Platz 6, D-20146 Hamburg, Germany

froeba@chemie.uni-hamburg.de

\section{SUPPORTING INFORMATION}




\section{Contents}

1 Abbreviations 4

2 Experimental Methods 4

2.1 Powder X-Ray Diffraction . . . . . . . . . . . . . . . . . . . . . . . . . . . 4

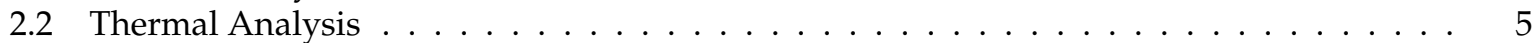

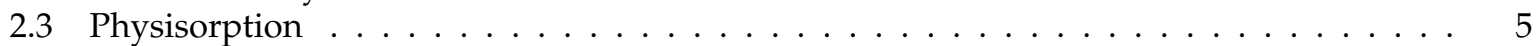

2.4 Characterization of Organic Reaction Products $\ldots \ldots \ldots \ldots \ldots$

2.5 Syntheses of the Linker Molecules $\quad \ldots \ldots \ldots \ldots \ldots \ldots$

3 Synthetic Procedures 9

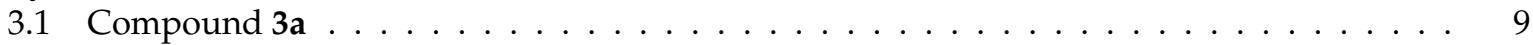

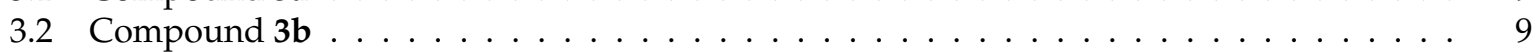

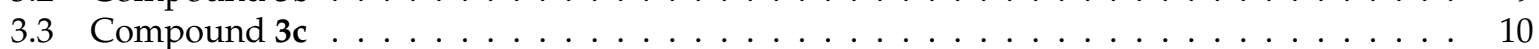

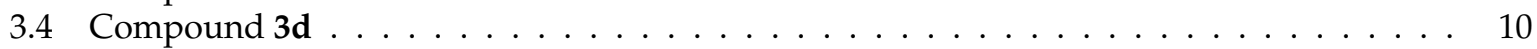

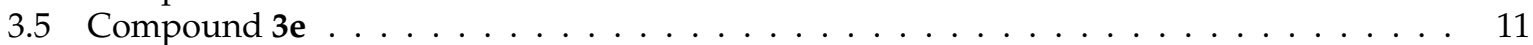

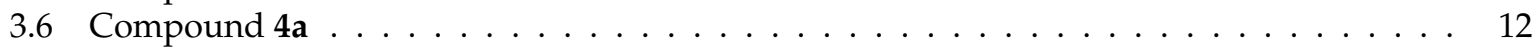

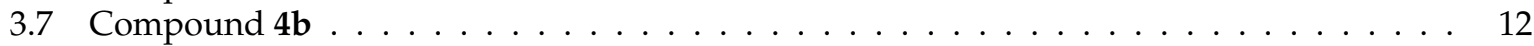

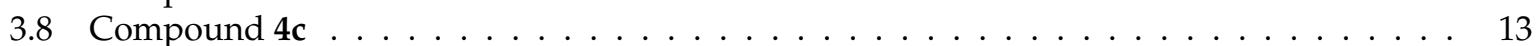

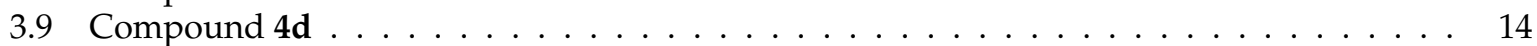

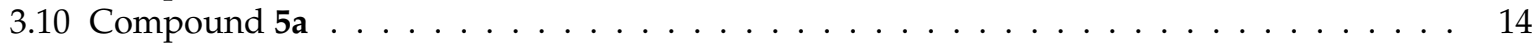

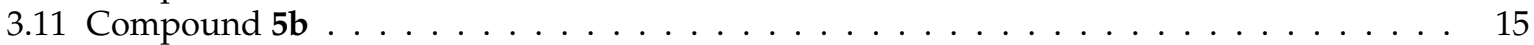

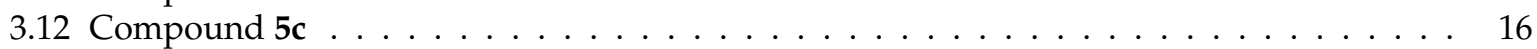

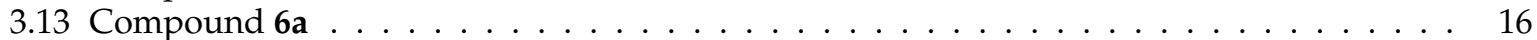

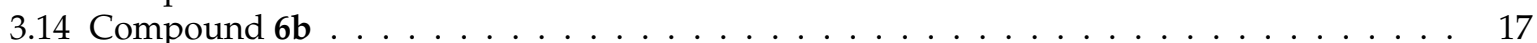

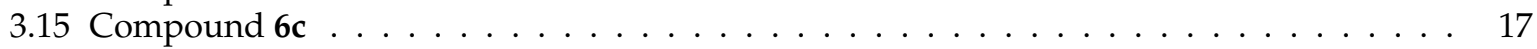

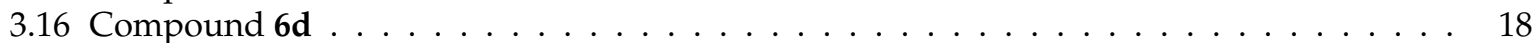

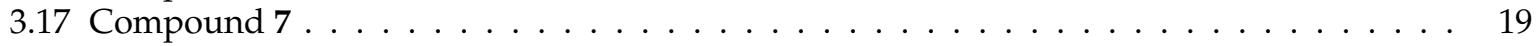

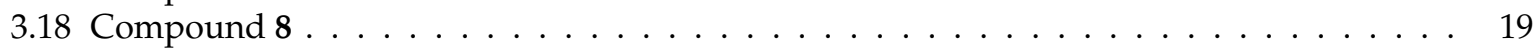

\begin{tabular}{|lll}
4 & Synthesis of the MOFs & 20
\end{tabular}

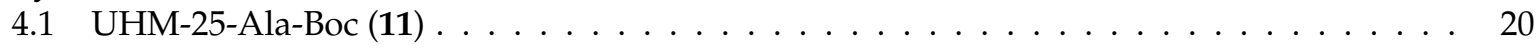

$4.1 .1 \quad$ Nitrogen Physisorption Measurement . . . . . . . . . . . . . . . . . . . . . . . 20

4.1 .2 Thermogravimetric Analysis $\ldots \ldots \ldots \ldots$. . . . . . . . . . . . . . . . . . . 21

4.1 .3 Crystal Structure Determination and Refinement . . . . . . . . . . . . . . . . . . . 21

4.1 .4 Investigations on the Structural Integrity of the Linker . . . . . . . . . . . . . 23

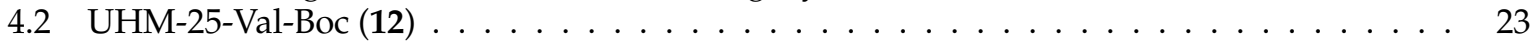

$4.2 .1 \quad$ Nitrogen Physisorption Measurement . . . . . . . . . . . . . . . . . . . . 23

4.2 .2 Thermogravimetric Analysis $\ldots \ldots \ldots \ldots \ldots$. . . . . . . . . . . . . . 24

4.3 UHM-25-Leu-Boc (13) $\ldots \ldots \ldots \ldots \ldots \ldots \ldots$. . . . . . . . . . . . . . . . . . . . 24

4.3 .1 Nitrogen Physisorption Measurement . . . . . . . . . . . . . . . . . . . . . . 25

4.3 .2 Thermogravimetric Analysis $\ldots \ldots \ldots \ldots \ldots$. . . . . . . . . . . . . 26

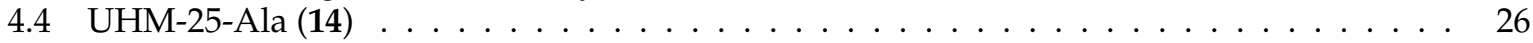

4.4 .1 Nitrogen Physisorption Measurement . . . . . . . . . . . . . . . . . . . . . . . 27

4.4 .2 Thermogravimetric Analysis $\ldots \ldots \ldots \ldots$. . . . . . . . . . . . . . . . . . . . . . 28

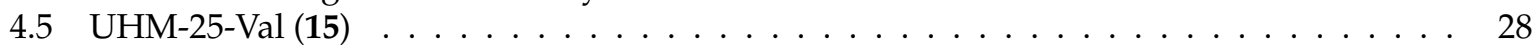

$4.5 .1 \quad$ Nitrogen Physisorption Measurement . . . . . . . . . . . . . . . . . . . . . . . . . . . . . . 29

4.5 .2 Thermogravimetric Analysis $\ldots \ldots \ldots$. . . . . . . . . . . . . . . . 30

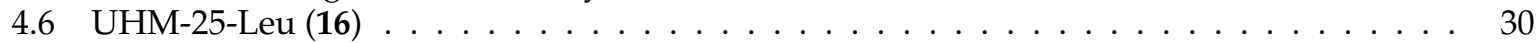

4.6 .1 Nitrogen Physisorption Measurement . . . . . . . . . . . . . . . . . . . . . . . . . 31

4.6 .2 Thermogravimetric Analysis $\ldots \ldots \ldots \ldots$. . . . . . . . . . . . . . . 32

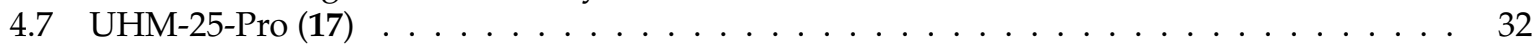

4.7 .1 Nitrogen Physisorption Measurement . . . . . . . . . . . . . . . . . . . . . 33

4.7 .2 Thermogravimetric Analysis $\ldots \ldots \ldots \ldots \ldots$. . . . . . . . . . . . . 34

4.7 .3 Crystal Structure Determination and Refinement . . . . . . . . . . . . . . . . . 35

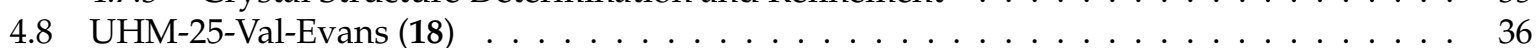

4.8 .1 Nitrogen Physisorption Measurement . . . . . . . . . . . . . . . . . . . . 36

4.8 .2 Thermogravimetric Analysis $\ldots \ldots \ldots \ldots$. . . . . . . . . . . . 37 
5 Catalysis and Post-Synthetic Modification 37

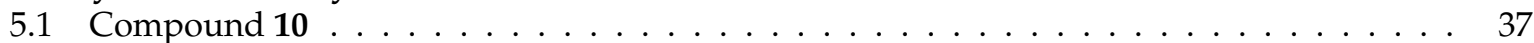

$5.2 \quad$ UHM-25-Val-Evans-PSM (20) . . . . . . . . . . . . . . . . . . . . . . . . . 38 


\section{Abbreviations}

AmOAc amyl acetate

ATR attenuated total reflection

Boc tert-butyloxycarbonyl

BET Brunauer-Emmet-Teller

Bn benzyl

DCVC dry column vacuum chromatography

DMAP 4-(dimethylamino)pyridine

DMF $N, N$-dimethylformamide

DMSO dimethylsulfoxide

DTA diferrential thermal analysis

EtOAc ethyl acetate

GC gas chromatography

HRMS high resolution mass spectrometry

MOF Metal-organic framework

MS mass spectrometry

NMR nuclear magnetic resonance

COSY correlated spectroscopy

PSM post-synthetic modification

PXRD powder X-ray diffraction

SBU secondary building unit

SIM selected ion monitoring

SPhos 2-dicyclohexylphosphino-2',6'-dimethoxybiphenyl

TFA trifluoroacetic acid

THF tetrahydrofuran

TLC thin layer chromatography

UHM University of Hamburg Materials

\section{Experimental Methods}

To investigate and prove the stability of the chiral linker after the MOF synthesis, the UHM-25 MOFs were digested in diluted hydrochloric acid. This allowed the reisolation of the linker which could then be examined by spectroscopic methods. The integrity of the linker was independently verified by ${ }^{1} \mathrm{H}-$ NMR spectroscopy, mass spectrometry and optical rotation.

\subsection{Powder X-Ray Diffraction}

Powder X-ray diffraction (PXRD) measurements of the MOFs were performed with the as-synthesized material before exchanging solvents, dried in air. Powder X-ray diffractograms were recorded on a STOE Stadi P by STOE \& Cie GmbH working in transmission geometry in the range of $2 \theta=0^{\circ}-70^{\circ}$. 


\subsection{Thermal Analysis}

TG/DTA/MS experiments were performed with a thermobalance STA 449 F3 Jupiter coupled with a Netzsch quadrupol mass spectrometer QMS 40 C Aëolos. All measurements were performed in a stream of an argon/oxygen mixture (80:20) with a flow rate of $20 \mathrm{ml} / \mathrm{min}$. Circa $20 \mathrm{mg}$ of sample were heated in an aluminium oxide crucible from room temperature to $800^{\circ} \mathrm{C}$ with a heating rate of $5 \mathrm{~K} / \mathrm{min}$.

\subsection{Physisorption}

Physisorption measurements were performed with a QUADRASORB SI-MP by Quantachrome GmbH \& Co KG with nitrogen at $77 \mathrm{~K}$. The determination of the specific surface area was performed on the basis of the BET micropore assistant provided in the Quantachrome ASiQwin software. MOFs were activated in vacuum after exchange with supercritical carbon dioxide for one hour at room temperature.

The exchange with supercritical carbon dioxide was carried out as follows: About $30 \mathrm{mg}$ of the material was subjected to a solvent exchange with amyl acetate, which was replaced two times with fresh solvent after 24 hours. After another 24 hours the solvent was decanted and the dark blue crystals were placed in a porous container. A solvent exchange was performed with liquid carbon dioxide at $10^{\circ} \mathrm{C}$ and 55 bar in a steel autoclave. The liquid carbon dioxide was replaced ten times in intervals of 6-18 hours. After the last exchange the autoclave was sealed and slowly heated to $35^{\circ} \mathrm{C}$ to reach the supercritical state of carbon dioxide. The autoclave was then slowly vented and the samples were kept under argon before nitrogen physisorption was measured. An overview of the specific surface areas of the UHM-25 MOFs that were obtained from physisorption measurements are given in Table 1. The individual sorption isotherms are shown for the respective MOFs in Section 4 .

Table 1 - Specific surface areas and micropore volumes of the UHM-25 MOFs determined after activation with supercritical carbon dioxide and calculated from the adsorption branch of the physisorption isotherms with their respective values for $p / p_{0}$.

\begin{tabular}{lrrrr}
\hline & \multicolumn{2}{c}{$S_{\text {BET }}$} & \multicolumn{2}{c}{$V_{\text {pore }}$} \\
& $\mathrm{m}^{2} \mathrm{~g}^{-1}$ & $p / p_{0}$ & $\mathrm{~cm}^{3} \mathrm{~g}^{-1}$ & $p / p_{0}$ \\
\hline UHM-25-Ala-Boc & 1474 & $0.016-0.100$ & 0.58 & 0.20 \\
UHM-25-Val-Boc & 1922 & $0.009-0.020$ & 1.00 & 0.20 \\
UHM-25-Leu-Boc & 1738 & $0.016-0.074$ & 0.68 & 0.20 \\
UHM-25-Ala & 975.7 & $0.010-0.048$ & 0.39 & 0.20 \\
UHM-25-Val & 1286 & $0.011-0.049$ & 0.51 & 0.20 \\
UHM-25-Leu & 1351 & $0.010-0.049$ & 0.53 & 0.20 \\
UHM-25-Pro & 371.5 & $0.010-0.048$ & 0.15 & 0.20 \\
UHM-25-Val-Evans & 1454 & $0.014-0.075$ & 0.56 & 0.20
\end{tabular}

\subsection{Characterization of Organic Reaction Products}

MS experiments were performed with a 6224 Accurate-Mass TOF-MS by Agilent Technologies. TLC was carried out on ALUGRAM Xtra SILG/UV 254 from MACHEREY-NAGEL. NMR measurements were performed at room temperature with a Fourier 300 from Bruker BioSpin GmbH. Spectra were referenced to the known chemical shifts of the residual solvents. ${ }^{1}$ In addition to one-dimensional ${ }^{1} \mathrm{H}$ and proton decoupled ${ }^{13} \mathrm{C}$ spectra, correlation spectroscopy (COSY), heteronuclear single quantum coherence (HSQC) and heteronuclear multiple bond correlation experiments were performed when necessary for an precise assignment of the NMR signals. IR spectroscopy was performed with potassium bromide disks or by attenuated total reflection (ATR) with a VERTEX 70 by Bruker. Elementary analysis was performed with a vario EL III by Elementar Analysensysteme GmbH and EuroEA Elemental Analyzer by HEKAtech GmbH. 


\subsection{Syntheses of the Linker Molecules}

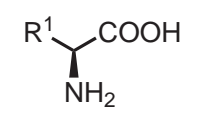

1a, $R^{1}=$ Me (alanine)

1b, $\mathrm{R}^{1}=i-\operatorname{Pr}$ (valine)

1c, $\mathrm{R}^{1}=i$-Bu (leucine)

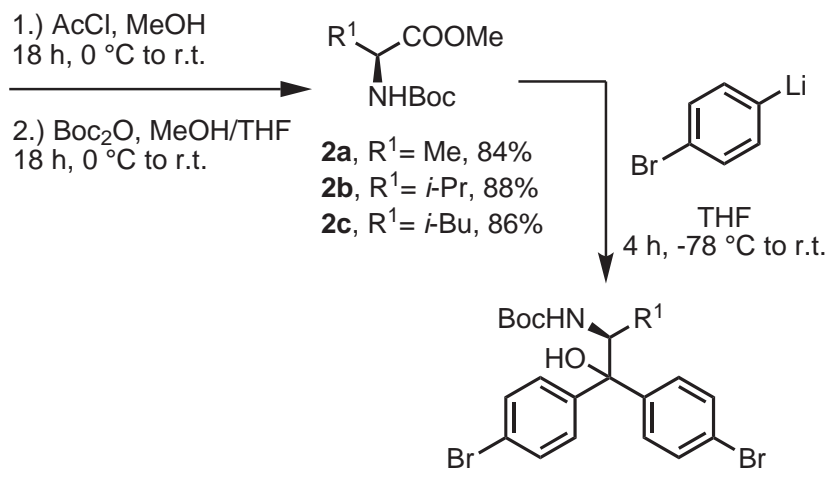

3a, $R^{1}=M e, 91 \%$

3b, $\mathrm{R}^{1}=i-\operatorname{Pr}, 93 \%$

3c, $\mathrm{R}^{1}=i-\mathrm{Bu}, 90 \%$

Figure S1 - Conversion of the amino acids to their protected derivatives and further conversion to the bisaryl substituted $\mathrm{N}$-Boc protected amino alcohol via nucleophilic substitution and addition to the carboxylic carbon atom of compounds $\mathbf{2 a - c}$; reactions were performed according to literature procedures.[2]

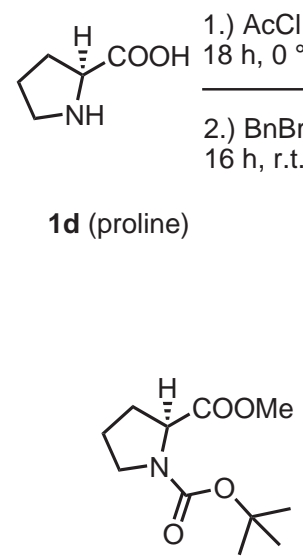

$2 e$<smiles>COC(=O)C1CCCN1Cc1ccccc1</smiles>

2d

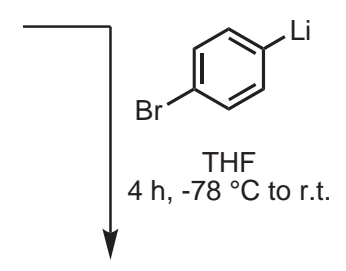

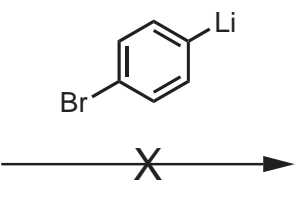

3d, $94 \%$

Figure S2 - Esterification, $N$-benzyl protection of proline (step 1) and the further conversion to the bisaryl substituted $\mathrm{N}$-Boc protected amino alcohol via nucleophilic substitution and addition to the carboxylic carbon atom of compound $\mathbf{2 d}$; a succesful reaction of the organolithium species with $\mathrm{N}$ Boc-proline methylester could not be achieved, hence the benzyl group was chosen to protect the secondary amine; reactions were performed according to literature procedures ${ }^{3}$ 
<smiles>[2H]C([NH+])C(c1ccc(Br)cc1)c1ccc(Br)cc1</smiles><smiles>COC(=O)c1cc(O)cc(C(=O)OCC(=O)O[Na])c1</smiles><smiles>[Y17]C([2H])C(O)(c1ccc(-c2cc(OC(C)=O)cc(C(C)=O)c2)cc1)c1ccc(-c2cc(OC(C)=O)cc(C(=O)OC)c2)cc1</smiles>
3a, $R^{1}=\mathrm{Me}, \mathrm{R}^{2}=\mathrm{Boc}, \mathrm{R}^{3}=\mathrm{H}$
3b, $\mathrm{R}^{1}=i-\operatorname{Pr}, \mathrm{R}^{2}=\mathrm{Boc}, \mathrm{R}^{3}=\mathrm{H}$
3c, $\mathrm{R}^{1}=i-\mathrm{Bu}, \mathrm{R}^{2}=\mathrm{Boc}, \mathrm{R}^{3}=\mathrm{H}$
$3 d, R^{1}, R^{3}=-\left(C_{2}\right)_{4}$ (cyclic), $R^{2}=B n$
4a, $R^{1}=M e, R^{2}=$ Boc, $R^{3}=H, 56 \%$
4b, $\mathrm{R}^{1}=i-\operatorname{Pr}, \mathrm{R}^{2}=\mathrm{Boc}, \mathrm{R}^{3}=\mathrm{H}, 70 \%$
4c, $\mathrm{R}^{1}=i-\mathrm{Bu}, \mathrm{R}^{2}=\mathrm{Boc}, \mathrm{R}^{3}=\mathrm{H}, 59 \%$
4d, $R^{1}, R^{3}=-\left(\mathrm{CH}_{2}\right)_{4}$ (cyclic), $R^{2}=\mathrm{Bn}, 64 \%$

Figure S3 - Suzuki-type cross-coupling reaction of the di(4-bromophenyl) substituted protected amino alcohols 2a-d with 3,5-(dimethoxycarbonyl)phenylboronic acid to synthesize the tetramethyl esters of the linker molecules (4a-d).

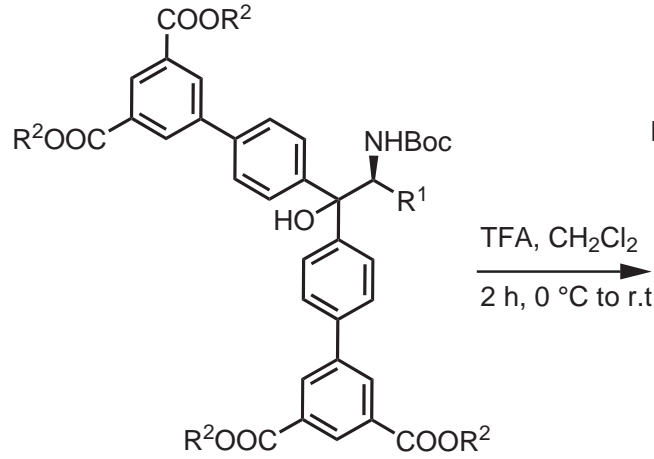

5a, $\mathrm{R}^{1}=\mathrm{Me}, \mathrm{R}^{2}=\mathrm{H}, 85 \%$

5b, $\mathrm{R}^{1}=i-\mathrm{Pr}, \mathrm{R}^{2}=\mathrm{H}, 80 \%$

5c, $\mathrm{R}^{1}=i-\mathrm{Bu}, \mathrm{R}^{2}=\mathrm{H}, 82 \%$

4a, $R_{1}=M e, R^{2}=M e$

4b, $\mathrm{R}_{1}=i-\operatorname{Pr}, \mathrm{R}^{2}=\mathrm{Me}$

4c, $\mathrm{R}_{1}=i-\mathrm{Bu}, \mathrm{R}^{2}=\mathrm{Me}$<smiles>N[C@@H](C([18O])C(O)(c1ccc(-c2cc(C(=O)O)cc(C(=O)O)c2)cc1)c1ccc(-c2cc(C(=O)O)cc(C(=O)O)c2)cc1)C(F)(F)F</smiles>

6a, $\mathrm{R}_{1}=\mathrm{Me}, 75 \%$

6b, $\mathrm{R}_{1}=i-\operatorname{Pr}, 72 \%$

$6 c, \mathrm{R}_{1}=i-\mathrm{Bu}, 80 \%$

Figure S4 - Deprotection procedure for the primary amino alcohols; First the tetramethyl esters 4a-c were hydrolyzed, then the $\mathrm{N}$-Boc group was cleaved acidolytically. 


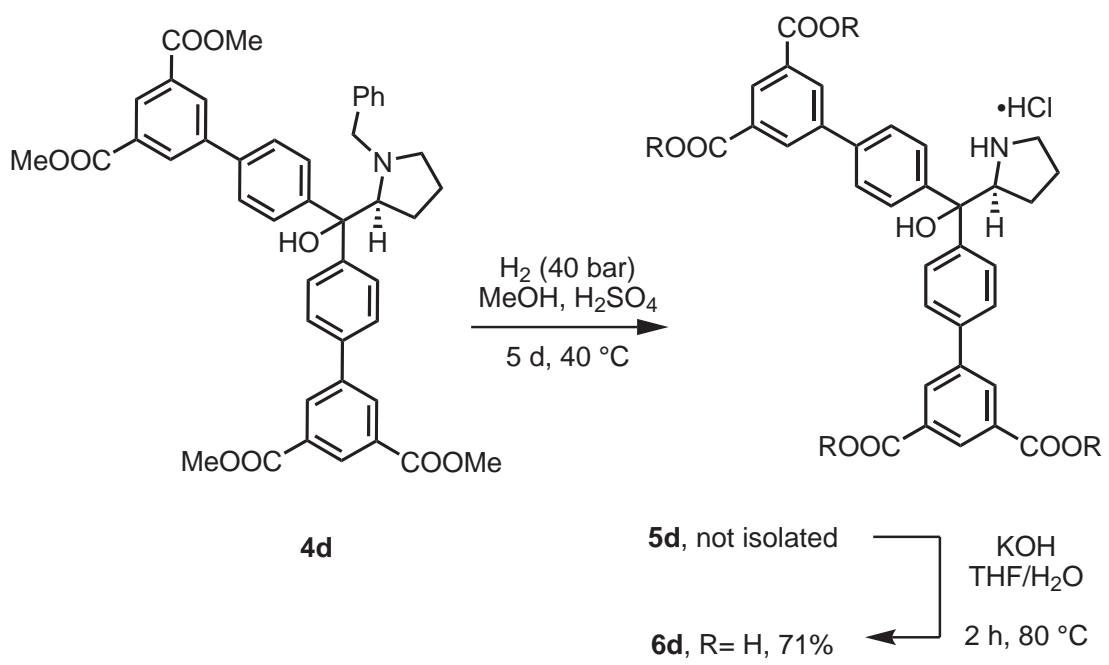

Figure S5 - Deprotection procedure for the secondary aminoalcohol: Cleavage of the $N$-benzyl substitutent preceded the hydrolyzation of the tetramethyl ester to obtain compound $\mathbf{6 d}$.

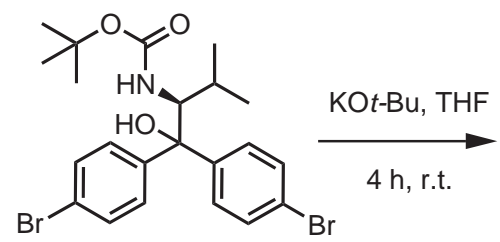

$3 b$<smiles>CC(C)[C@H]1NC(=O)OC1(c1ccc(Br)cc1)c1ccc(Br)cc1</smiles>

3e (quant.)

Figure S6 - Intramolecular cyclization of the $\mathrm{N}$-Boc protected derivative of valine to obtain the di(4bromophenyl) substituted 1,3-oxazolidin-2-one (3e), which is a derivative of the so-called Evansauxiliary.<smiles>CC(C)[C@H]1NC(=O)OC1(c1ccc(Br)cc1)c1ccc(Br)cc1</smiles>

$3 e$<smiles>COC(=O)c1cc([18OH])cc(C(=O)OC)c1</smiles>

$\left[\mathrm{Pd}\left(\mathrm{PPh}_{3}\right)_{4}\right], \mathrm{Cs}_{2} \mathrm{CO}_{3}$ SPhos

$\mathrm{MeOH} / \mathrm{DMF}$ $4 \mathrm{~h}, 80^{\circ} \mathrm{C}$
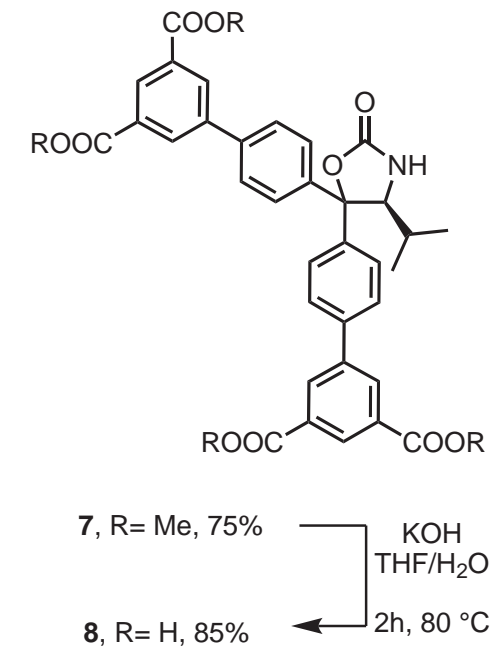

Figure S7 - Suzuki cross-coupling reaction of the di(4-bromophenyl) substituted 1,3-oxazolidin-2one (3e) and the subsequent hydrolysis of the tetramethyl ester 7. 


\section{Synthetic Procedures}

\subsection{1,1-Di(4-bromophenyl)-N-tert-butoxycarbonyl alaninol (3a)}

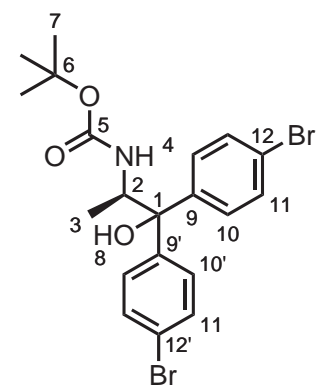

The reactions were performed in oven-dried glassware under argon. A solution of 1,4-dibromobenzene $(60.8 \mathrm{~g}, 258 \mathrm{mmol}, 3.6 \mathrm{eq})$ in THF $(375 \mathrm{~mL})$ was cooled to $-78^{\circ} \mathrm{C}$. A solution of $n$-butyllithium in hexane $(2.5 \mathrm{M}, 100 \mathrm{~mL}, 258 \mathrm{mmol}, 3.6 \mathrm{eq})$ was added over thirty minutes. The reaction solution was stirred for another sixty minutes at $-78^{\circ} \mathrm{C}$. To the resulting colorless suspension of 4-bromophenyllithium a solution of the $N$-protected amino acid methylester 2a (14.5 g, $71.4 \mathrm{mmol}, 1 \mathrm{eq}$.) in THF (15.0 mL) was added dropwise. The reaction solution was stirred for another 60 minutes and was slowly warmed to room temperature. The reaction solution was monitored by TLC. Upon consumption of compound $2 \mathbf{a}$, the reaction solution was quenched by the addition of a saturated solution of $\mathrm{NH}_{4} \mathrm{Cl}$ in water $(500 \mathrm{~mL})$. The reaction mixture was extracted with EtOAc $(750 \mathrm{~mL})$. Two phases were separated and the aqueous phase was extracted with EtOAc again $(2 \times 150 \mathrm{~mL})$. The combined organic phases were washed with aqueous $\mathrm{HCl}(1 \mathrm{M}, 2 \times 250 \mathrm{~mL})$, a saturated solution of $\mathrm{NaHCO}_{3}(2 \times 250 \mathrm{~mL})$ and a saturated solution of $\mathrm{NaCl}(2 \times 250 \mathrm{~mL})$. The organic phase was dried over $\mathrm{Na}_{2} \mathrm{SO}_{4}$ and the solvent was evaporated under reduced pressure. The crude reaction product was purified by dry column vacuum chromatography $(\mathrm{DCVC}) \underline{\underline{4}}$

Yield: $31.4 \mathrm{~g}$ (65.0 mmol, 91\%); colorless crystals; $\mathbf{M : ~}\left[\mathrm{C}_{20} \mathrm{H}_{23} \mathrm{Br}_{2} \mathrm{NO}_{3}\right] 484.0 \frac{\mathrm{g}}{\mathrm{mol}} ;$ TLC: $R_{f}=0.45$ (heptane/EtOAc, 4:1); opt. rot.: $[\alpha]_{\mathrm{D}}^{27}=-30.6$ (c=0.58, MeOH); HRMS (ESI+): $\frac{m}{z}=505.9956$ (calc. for $\mathrm{MNa}^{+}$: 505.9942). elem. anal.: \% = C (49.82), $\mathrm{H}(4.83), \mathrm{Br}$ (n.d.), $\mathrm{N}(2.54) \mathrm{O}$ (9.99) calc. for $\mathrm{C}_{20} \mathrm{H}_{23} \mathrm{Br}_{2} \mathrm{NO}_{3}$ : C (49.51), $\mathrm{H}(4.78), \mathrm{Br}(32.94), \mathrm{N}(2.89), \mathrm{O}$ (9.89).

${ }^{1}$ H-NMR (300 MHz, DMSO-d $\left.\mathbf{d}_{6}\right): \delta[\mathrm{ppm}]=7.48-7.39(\mathrm{~m}, 8 \mathrm{H}, \mathrm{H} 10, \mathrm{H} 11) 6.44\left(\mathrm{~d}, 1 \mathrm{H},{ }^{3} J_{\mathrm{H}-\mathrm{H}}=9.5 \mathrm{~Hz}\right.$, $\mathrm{H} 4), 5.72(\mathrm{~s}, 1 \mathrm{H}, \mathrm{H} 8), 4.63\left(\mathrm{dq}, 1 \mathrm{H},{ }^{3} J_{\mathrm{H}-\mathrm{H}}=9.5,6.5 \mathrm{~Hz}, \mathrm{H} 2\right), 1.30(\mathrm{~s}, 9 \mathrm{H}, \mathrm{H} 7), 0.88\left(\mathrm{~d}, 3 \mathrm{H},{ }^{3} J_{\mathrm{H}-\mathrm{H}}=6.5 \mathrm{~Hz}\right.$ $\mathrm{H} 3)$.

${ }^{13} \mathrm{C}-\mathrm{NMR}\left(75 \mathrm{MHz}, \mathrm{DMSO}-\mathbf{d}_{6}\right): \delta$ [ppm] = $154.8(1 \mathrm{C}, \mathrm{C} 5), 145.6,144.8(2 \mathrm{C}, \mathrm{C} 12), 131.0,130.6,128.2$, 127.5 (4C, C10, C11), 119.7, 119.6 (2C, C9), 79.2 (1C, C1), 78.0 (1C, C6), 51.4 (1C, C2), 28.1 (3C, C7), 15.6 $(1 \mathrm{C}, \mathrm{C} 3)$.

IR (ATR): $\widetilde{v}\left[\mathrm{~cm}^{-1}\right]=3411(\mathrm{~m}), 3083(\mathrm{w}), 2968(\mathrm{w}), 2933(\mathrm{w}), 2869(\mathrm{w}), 2850(\mathrm{w}), 1672(\mathrm{~s}), 1515(\mathrm{~s}), 1490$ (m), 1454 (m), 1398 (m), 1357 (s), 1247 (m), 1172 (s), 1157 (s), 1074 (m), 1060 (s), 1008 (s), 900 (m), 811 (s), 765 (s).

\subsection{1,1-Di(4-bromophenyl)-N-tert-butoxycarbonyl valinol (3b)}

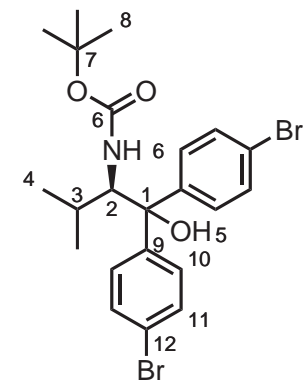

The reaction was performed according to the procedure described in Section 3.1 
Yield: $20.3 \mathrm{~g}$ (34.1 mmol, 93\%); colorless crystals; $\mathrm{M}:\left[\mathrm{C}_{22} \mathrm{H}_{27} \mathrm{Br}_{2} \mathrm{NO}_{3}\right] 513.3 \frac{\mathrm{g}}{\mathrm{mol}} ;$ TLC: $R_{f}=0.49$ (heptane/ EtOAc, 4:1); m.p.: $214^{\circ} \mathrm{C}$; opt. rot.: $[\alpha]_{\mathrm{D}}^{27}=-36.4(\mathrm{c}=0.85, \mathrm{MeOH})$; HRMS (ESI+): $\frac{\mathrm{m}}{z}=$ 534.0255 (calc. for $\left.\mathrm{MNa}^{+}: 534.0258\right)$. elem. anal.: \% = C (51.70), $\mathrm{H}(5.36), \mathrm{Br}$ (n.d.), $\mathrm{N}(2.48), \mathrm{O}$ (9.69), calc. for $\mathrm{C}_{10} \mathrm{H}_{11} \mathrm{BO}_{6}$ : C (51.48), $\mathrm{H}$ (5.30), $\mathrm{Br}$ (n.d.), $\mathrm{N}(2.73), \mathrm{O}$ (9.35).

${ }^{1}$ H-NMR (300 MHz, $\left.\mathrm{CDCl}_{3}\right): \delta$ [ppm] = 7.54-7.37 (m, 8H; H11, H11', H12, H12'), $5.96\left(\mathrm{~d}, 1 \mathrm{H},{ }^{3} \mathrm{~J}_{\mathrm{H}-\mathrm{H}}=\right.$ $10.4 \mathrm{~Hz}, \mathrm{H6}), 5.69$ (s, 1H, H5), $4.48\left(\mathrm{dd}, 1 \mathrm{H}, 3 J_{\mathrm{H}-\mathrm{H}}=10.4,2.3 \mathrm{~Hz}, \mathrm{H} 2\right), 1.60(\mathrm{~m}, 1 \mathrm{H}, \mathrm{H} 3), 1.31$ (s, 9H; H9), $0.84\left(\mathrm{~d}, 3 \mathrm{H},{ }^{3} \mathrm{~J}_{\mathrm{H}-\mathrm{H}}=6.6 \mathrm{~Hz}, \mathrm{H} 4 \mathrm{~A}\right), 0.71\left(\mathrm{~d}, 3 \mathrm{H},{ }^{3} \mathrm{~J}_{\mathrm{H}-\mathrm{H}}=6.9 \mathrm{~Hz}, \mathrm{H} 4 \mathrm{~B}\right)$.

${ }^{13}$ C-NMR (75 MHz, DMSO-d d $\left._{6}\right): \delta$ [ppm] = $156.3(1 \mathrm{C}, \mathrm{C} 7), 147.3,145.5$ (2C, C13, C13'), 131.5, 131.1, 128.4, 127.8 (4C; C11, C11', C12, C12'), 120.1, 120.0 (2C; C10, C10'), 81.2 (1C, C1), 78.5 (1C, C8), 59.5 (1C, C2), 28.7 (1C, C3), 28.6 (3C, C9), 23.3, 18.4 (2C, C4A, C4B).

IR (KBr): $\widetilde{v}\left[\mathrm{~cm}^{-1}\right]=3431(\mathrm{~m}), 3420(\mathrm{~m}), 2997(\mathrm{~m}), 2964(\mathrm{~m}), 2934(\mathrm{~m}), 2874(\mathrm{~m}), 1676(\mathrm{~s}), 1516(\mathrm{~s}), 1487$ (s), $1396(\mathrm{~m}), 1367(\mathrm{~s}), 1313(\mathrm{~m}), 1250(\mathrm{~s}), 1169(\mathrm{~s}), 1097(\mathrm{~m}), 1074(\mathrm{~m}), 1042(\mathrm{w}), 1011(\mathrm{~s}), 997(\mathrm{~m}), 934(\mathrm{w})$, $907(\mathrm{~m}), 868(\mathrm{~m}), 814(\mathrm{~m}), 783(\mathrm{~m}), 766(\mathrm{~m}), 725(\mathrm{w}), 681(\mathrm{w}), 613(\mathrm{w}), 546(\mathrm{~m}), 521(\mathrm{~m}), 507(\mathrm{~m}), 480(\mathrm{~m})$.

\subsection{1,1-Di(4-bromophenyl)-N-tert-butoxycarbonyl leucinol 3c}

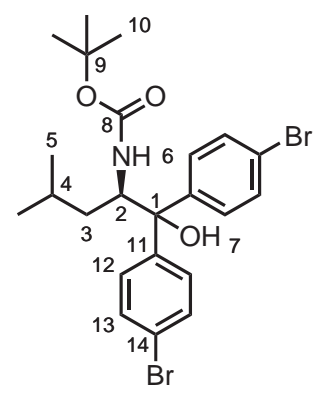

The reaction was performed according to the procedure described in Section 3.1

Yield: $20.3 \mathrm{~g}$ (38.7 mmol, 90\%); colorless crystals; $\mathrm{M}:\left[\mathrm{C}_{23} \mathrm{H}_{29} \mathrm{Br}_{2} \mathrm{NO}_{3}\right] 527.3 \frac{\mathrm{g}}{\mathrm{mol}} ; \mathrm{TLC}: R_{f}=0.47$ (heptane/EtOAc, 4:1); opt. rot.: $[\alpha]_{\mathrm{D}}^{27}=-6.4(\mathrm{c}=0.78, \mathrm{MeOH})$; HRMS (ESI+): $\frac{m}{z}=548.0406$ (calc. for $\mathrm{MNa}^{+}$: 548.0412). elem. anal.: \% = C (53.02), $\mathrm{H}$ (5.86), $\mathrm{Br}$ (n.d.), $\mathrm{N}(2.62), \mathrm{O}(10.04)$ calc. for $\mathrm{C}_{23} \mathrm{H}_{29} \mathrm{Br}_{2} \mathrm{NO}_{3}$ : C (52.39), $\mathrm{H}(5.54), \mathrm{Br}$ (30.31), $\mathrm{N}$ (2.66), O (9.10).

${ }^{1} \mathrm{H}-\mathrm{NMR}\left(300 \mathrm{MHz}, \mathrm{CDCl}_{3}\right): \delta$ [ppm] = 7.48-7.29 (m, 8H, H12, H13, H12', H13') $4.70\left(\mathrm{~d}, 1 \mathrm{H},{ }^{3} J_{\mathrm{H}-\mathrm{H}}=\right.$ $9.7 \mathrm{~Hz}, \mathrm{H} 6), 5.72(\mathrm{~s}, 1 \mathrm{H}, \mathrm{H} 8), 4.56\left(\mathrm{t}, 1 \mathrm{H},{ }^{3} \mathrm{~J}_{\mathrm{H}-\mathrm{H}}=10.4 \mathrm{~Hz}, \mathrm{H} 2\right), 3.72(\mathrm{~s}, \mathrm{H} 7), 1.75-1.58(\mathrm{~m}, 1 \mathrm{H}, \mathrm{H} 4), 1.37$ $\left(\mathrm{dd}, 1 \mathrm{H},{ }^{3} J_{\mathrm{H}-\mathrm{H}}=10.9,3.0 \mathrm{~Hz}, \mathrm{H} 3 \mathrm{~A}\right), 1.29(\mathrm{~s}, 9 \mathrm{H}, \mathrm{H} 10), 1.18-1.03(\mathrm{~m}, 1 \mathrm{H}, \mathrm{H} 3 \mathrm{~B}), 0.95\left(\mathrm{~d}, 3 \mathrm{H},{ }^{3} J_{\mathrm{H}-\mathrm{H}}=6.5\right.$ $\mathrm{Hz}, \mathrm{H} 5 \mathrm{~A}) 0.86\left(\mathrm{~d}, 3 \mathrm{H},{ }^{3} \mathrm{~J}_{\mathrm{H}-\mathrm{H}}=6.7 \mathrm{~Hz}, \mathrm{H} 5 \mathrm{~B}\right)$.

${ }^{13} \mathrm{C}-\mathrm{NMR}\left(75 \mathrm{MHz}, \mathrm{DMSO}-\mathbf{d}_{6}\right): \delta$ [ppm] = $156.2(1 \mathrm{C}, \mathrm{C} 8), 144.0,143.6$ (2C, C14, C14'), 131.6, 131.1, 127.8, 127.4 (4C, C12, C12', C13, C13'), 121.2, 121.0 (2C, C11, C11'), 81.1 (1C, C1), 79.7 (1C, C9), 54.9 (1C, C2), 39.2 (1C, C3), 28.2 (3C, C10), 25.2 (1C, C4), 23.9, 21.5 (2C, C5A, C5B).

IR (ATR): $\widetilde{v}\left[\mathrm{~cm}^{-1}\right]=3415(\mathrm{~m}), 2979(\mathrm{w}), 2954(\mathrm{w}), 2869(\mathrm{w}), 1672(\mathrm{~s}), 1521(\mathrm{~s}), 1488(\mathrm{~m}), 1450(\mathrm{~m}), 1363$ (m), $1267(\mathrm{~m}), 1170(\mathrm{~m}), 1159(\mathrm{~m}), 1076(\mathrm{~m}), 1008(\mathrm{~m}), 902(\mathrm{~m}), 819(\mathrm{~m}), 811(\mathrm{~m}), 771(\mathrm{~m})$.

\subsection{1,1-Di(4-bromophenyl)-N-benzyl prolinol (3d)}

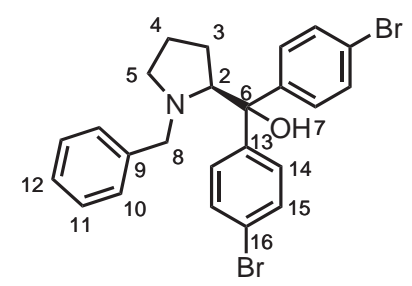

The reaction was performed according to the procedure described in Section 3.1

Yield: $20.3 \mathrm{~g}$ (33.8 mmol, 94\%); yellow oil; $\mathrm{M}:\left[\mathrm{C}_{24} \mathrm{H}_{23} \mathrm{Br}_{2} \mathrm{NO}\right] 499.0 \frac{\mathrm{g}}{\mathrm{mol}} ; \mathrm{TLC}: R_{f}=0.49$ (heptane/EtOAc, 4:1); opt. rot.: $[\alpha]_{\mathrm{D}}^{27}=66.6$ (c =0.74, MeOH); HRMS (ESI+): $\frac{m}{z}=500.0258$ (calc. for $\left.\mathrm{MH}^{+}: 500.0219\right)$

elem. anal.: \% = C (57.81), $\mathrm{H}(4.79), \mathrm{Br}$ (n.d.), $\mathrm{N}(2.61), \mathrm{O}$ (3.61), calc. for $\mathrm{C}_{24} \mathrm{H}_{23} \mathrm{Br}_{2} \mathrm{NO}$ : C (57.51), $\mathrm{H}$ (4.62), $\mathrm{Br}(31.88), \mathrm{N}(2.79), \mathrm{O}(3.19)$. 
${ }^{1}$ H-NMR $\left(300 \mathrm{MHz}, \mathrm{CDCl}_{3}\right): \delta$ [ppm] = 7.64-7.56 (m, 2H, H14), 7.48-7.38 (m, 6H, H14', H15, H15'), 7.35-7.18 (m, 3H, H10, H12), 7.10-7.02 (m, 2H, H11), 5.07 (s, 1H, H7), $3.92\left(\mathrm{dd}, 1 \mathrm{H},{ }^{3} \mathrm{~J}_{\mathrm{H}-\mathrm{H}}=9.2,4.3 \mathrm{~Hz}\right.$, $\mathrm{H} 2), 3.33\left(\mathrm{~d}, 1 \mathrm{H},{ }^{2} J_{\mathrm{H}-\mathrm{H}}=12.7 \mathrm{~Hz}, \mathrm{H} 8 \mathrm{~A}\right), 3.10\left(\mathrm{~d}, 1 \mathrm{H},{ }^{2} J_{\mathrm{H}-\mathrm{H}}=12.7 \mathrm{~Hz}, \mathrm{H} 8 \mathrm{~B}\right), 2.95\left(\mathrm{ddd}, 1 \mathrm{H},{ }^{2} J_{\mathrm{H}-\mathrm{H}}=8.1\right.$ $\left.\mathrm{Hz},{ }^{3} J_{\mathrm{H}-\mathrm{H}}=4.9,3.3 \mathrm{~Hz}, \mathrm{H} 5 \mathrm{~A}\right), 2.40\left(\mathrm{dt}, 1 \mathrm{H}, 1 \mathrm{H},{ }^{2} J_{\mathrm{H}-\mathrm{H}}=8.1 \mathrm{~Hz},{ }^{3} J_{\mathrm{H}-\mathrm{H}}=9.5 \mathrm{~Hz}, \mathrm{H} 5 \mathrm{~B}\right), 2.03-1.84(\mathrm{~m}, 1 \mathrm{H}$, H3A), 1.77-1.57 (m, 3H, H3B, H4).

${ }^{13}$ C-NMR (75 MHz, DMSO- $\left.\mathbf{d}_{6}\right): \delta$ [ppm] = 146.9, $145.4\left(2 \mathrm{C}, \mathrm{C} 16, \mathrm{C} 16{ }^{\prime}\right), 139.2$ (1C, C9), 131.4, 131.3 (2C, C15, C15'), 128.5,128.3 (4C, C10, C11), 127.4, 127.2 (2C, C14, C14'), 127.1 (1C, C12), 120.7, 120.4 (2C, C13, C13'), 70.2 (1C, C2), 60.5 (1C, C8), 55.5 (1C, C5), 29.8 (1C, C3), 24.0 (1C, C4).

IR (KBr): $\widetilde{v}\left[\mathrm{~cm}^{-1}\right]=3313(\mathrm{br}), 3083(\mathrm{w}), 3062(\mathrm{w}), 3029,(\mathrm{w}), 2952(\mathrm{w}), 2921(\mathrm{w}), 2869(\mathrm{w}), 2802(\mathrm{w})$, $1587(\mathrm{w}), 1485$ (s), $1452(\mathrm{~m}), 1398(\mathrm{~m}), 1373(\mathrm{~m}), 1243(\mathrm{~m}), 1178(\mathrm{~m}), 1097(\mathrm{~m}), 1072(\mathrm{~s}), 1008(\mathrm{~s}), 840(\mathrm{~m})$, $804(\mathrm{~s}), 698(\mathrm{~s})$.

\subsection{4,4-Di(4-bromophenyl)-5-isopropyl-oxazolidin-2-one (3e)}<smiles>[3H]C(C)C1NC(=O)OC1(c1ccc(Br)cc1)c1ccc(Br)cc1</smiles>

A solution of the $N$-Boc-protected amino alcohol $3 \mathbf{b}(4.50 \mathrm{~g}, 8.77 \mathrm{mmol}, 1 \mathrm{eq}$.$) in THF (90 \mathrm{~mL})$ was cooled to $0{ }^{\circ} \mathrm{C}$. Potassium tert-butoxide $(1.18 \mathrm{~g}, 10.5 \mathrm{mmol}, 1.2$ eq. $)$ was added. The reaction solution was allowed to warm to room temperature and was stirred for another three hours. The reaction solution was monitored by TLC. Upon consumption of compound $\mathbf{3 b}$, the reaction mixture was diluted with EtOAc $(250 \mathrm{~mL})$ and extracted with a saturated solution of $\mathrm{NH}_{4} \mathrm{Cl}$ in water $(100 \mathrm{~mL})$. Two phases were separated and the aqueous phase was extracted with EtOAc again $(2 \times 100 \mathrm{~mL})$ The combined organic phases were washed with a saturated solution of $\mathrm{NaCl}(2 \times 100 \mathrm{~mL})$, dried over $\mathrm{Na}_{2} \mathrm{SO}_{4}$ and the solvent was evaporated under reduced pressure.

Yield: $3.81 \mathrm{~g}$ (8.73 mmol, quant.); colorless oil (crystallized upon cooling); $\mathbf{M}:\left[\mathrm{C}_{18} \mathrm{H}_{17} \mathrm{Br}_{2} \mathrm{NO}_{2}\right] 484.0 \frac{\mathrm{g}}{\mathrm{mol}}$; m.p.: $119^{\circ} \mathrm{C}$; TLC: $R_{f}=0.56$ (heptane/EtoAC, 1:1); opt. rot.: $[\alpha]_{\mathrm{D}}^{27}=-137.0(\mathrm{c}=0.70$, MeOH); HRMS (ESI+): $\frac{m}{z}=459.9524$ (calc. for $\mathrm{MNa}^{+}$: 459.9513); elem. anal.: \% = C (50.31), $\mathrm{H}$ (4.22), $\mathrm{Br}$ (n.d.), $\mathrm{N}(2.80)$, $\mathrm{O}$ (9.07) calc. for $\mathrm{C}_{18} \mathrm{H}_{17} \mathrm{Br}_{2} \mathrm{NO}_{2}$ : C (49.23), $\mathrm{H}$ (3.90), $\mathrm{Br}$ (36.39), $\mathrm{O}$ (7.29).

${ }^{1} \mathrm{H}-\mathrm{NMR}\left(300 \mathrm{MHz}, \mathrm{CDCl}_{3}\right): \delta[\mathrm{ppm}]=8.21(\mathrm{~s}, 1 \mathrm{H}, \mathrm{H1}), 7.68-7.38\left(\mathrm{~m}, 8 \mathrm{H}, \mathrm{H} 9, \mathrm{H}^{\prime}, \mathrm{H} 10, \mathrm{H}^{\prime} 0^{\prime}\right), 4.41$ $\left(\mathrm{dd}, 1 \mathrm{H},{ }^{3} J_{\mathrm{H}-\mathrm{H}}=2.2,1.0 \mathrm{~Hz}, \mathrm{H} 5\right), 1.83-1.70(\mathrm{~m}, 1 \mathrm{H}, \mathrm{H6}), 0.90\left(\mathrm{~d}, 3 \mathrm{H},{ }^{3} \mathrm{~J}_{\mathrm{H}-\mathrm{H}}=6.9 \mathrm{~Hz}, \mathrm{H} 6 \mathrm{~A}\right), 0.49(\mathrm{~d}, 3 \mathrm{H}$; $\left.{ }^{3} J_{\mathrm{H}-\mathrm{H}}=6.6 \mathrm{~Hz}, \mathrm{H} 6 \mathrm{~B}\right)$.

${ }^{13} \mathrm{C}-\mathrm{NMR}\left(75 \mathrm{MHz}, \mathrm{CDCl}_{3}\right): \delta$ [ppm] = $157.3(1 \mathrm{C}, \mathrm{C} 2), 144.7,139.2\left(2 \mathrm{C} ; \mathrm{C} 11, \mathrm{C} 11^{\prime}\right), 131.9,131.6(4 \mathrm{C}$, C10, C10'), 128.9, 128.2 (4C, C9, C9'), 121.7, 121.2 (2C, C8, C8'), 87.4 (1C, C4), 60.2 (1C; C5), 29.5 (1C, C6), $21.2(1 \mathrm{C}, \mathrm{C} 6 \mathrm{~A}), 14.8(1 \mathrm{C}, \mathrm{C} 6 \mathrm{~B})$.

IR (KBr): $\widetilde{v}\left[\mathrm{~cm}^{-1}\right]=2955(\mathrm{~m}), 1767(\mathrm{~s}), 1726(\mathrm{~s}), 1601(\mathrm{~m}), 1514(\mathrm{w}), 1448(\mathrm{~m}), 1435(\mathrm{~m}), 1396(\mathrm{~m}), 1379$ (m), $1344(\mathrm{~m}), 1246(\mathrm{~s}), 1196(\mathrm{~m}), 1132(\mathrm{~m}), 1109(\mathrm{w}), 1090(\mathrm{w}), 1072(\mathrm{~m}), 1059(\mathrm{w}), 1026(\mathrm{w}), 999(\mathrm{~m}), 962$ (m), $930(\mathrm{w}), 910(\mathrm{w}), 833(\mathrm{~m}), 756(\mathrm{~s}), 733(\mathrm{~m}), 719(\mathrm{~m}), 704(\mathrm{~m}), 683(\mathrm{~m}), 646(\mathrm{w}), 635(\mathrm{w}), 606(\mathrm{w}), 523$ $(\mathrm{w})$. 


\subsection{Di-(O,O-dimethyl-3', 5' -dicarboxybiphenyl)- $N$-tert-butyloxycarbonyl-alaninol (4a)}<smiles>COC(=O)c1cc(C(=O)OC)cc(-c2ccc(C(O)(c3ccc(-c4cc(C(=O)OC)cc([13C](=O)OC)c4)cc3)[C@](C)(O)NC(=O)OC(C)(C)C)cc2)c1</smiles>

The reaction was performed in oven-dried glassware under argon. Solvents were degassed by three freeze-pump-thaw cycles prior to use. To a suspension of the bis-arylated amino acid derivative 3a (3.00 g, $6.21 \mathrm{mmol}, 1$ eq.), 3,5-(dimethoxycarbonyl)phenylboronic acid (4.44 g, $18.6 \mathrm{mmol}, 3.0$ eq.) and cesium carbonate $\left(\mathrm{Cs}_{2} \mathrm{CO}_{3}, 6.06 \mathrm{~g}, 18.6 \mathrm{mmol}, 3.0\right.$ eq.) in $\mathrm{MeOH}(100 \mathrm{~mL})$ and $\mathrm{DMF}(25 \mathrm{~mL})$ tetrakis triphenyl phosphine ([Pd( $\left.\left.\mathrm{PPh}_{3}\right)_{4}\right], 483 \mathrm{mg}, 0.31 \mathrm{mmol}, 0.05$ eq.) and 2-dicyclohexylphosphino-2',6'-dimethoxybiphenyl (SPhos) (127 mg, $0.31 \mathrm{mmol}, 0.05 \mathrm{eq}$.) were added. The reaction solution was heated to reflux for four hours and was monitored by TLC. Upon consumption of compound 3a, the reaction mixture was allowed to cool to room temperature. The reaction mixture was concentrated in vacuum to remove most of the MeOH and DMF. The residue was diluted in a mixture of EtOAc (400 mL) and water $(200 \mathrm{~mL})$. The organic phase was separated and washed with a saturated solution of $\mathrm{NaHCO}_{3}$ $(2 \times 100 \mathrm{~mL})$ and a saturated solution of $\mathrm{NaCl}(2 \times 100 \mathrm{~mL})$. The organic phase was dried over $\mathrm{Na}_{2} \mathrm{SO}_{4}$ and the solvent was evaporated under reduced pressure. The crude reaction product was purified by DCVC!

Yield: $2.50 \mathrm{~g}$ (3.51 mmol, 56\%); yellow foam; M: $\left[\mathrm{C}_{40} \mathrm{H}_{41} \mathrm{NO}_{11}\right] 711.8 \frac{\mathrm{g}}{\mathrm{mol}} ;$ TLC: $R_{f}=0.19$ (heptane/EtOAc, 2:1); opt. rot.: $[\alpha]_{\mathrm{D}}^{27}=-9.4$ (c $\left.=0.68, \mathrm{MeOH}\right)$; HRMS (ESI+): $\frac{m}{z}=734.2614$ (calc. for $\mathrm{MNa}^{+}$: 734.2577) elem. anal.: $\%=\mathrm{C}(67.62), \mathrm{H}(6.30), \mathrm{N}(1.59), \mathrm{O}(23.48)$, calc. for $\mathrm{C}_{40} \mathrm{H}_{41} \mathrm{NO}_{11}: \mathrm{C}$ (67.50), H (5.81), N (1.97), O (24.73) .

${ }^{1} \mathrm{H}-\mathrm{NMR}\left(300 \mathrm{MHz}, \mathrm{CDCl}_{3}\right): \delta[\mathrm{ppm}]=8.63(\mathrm{~m}, 2 \mathrm{H}, \mathrm{H} 16), 8.43\left(\mathrm{~d}, 2 \mathrm{H},{ }^{4} J_{\mathrm{H}-\mathrm{H}}=1.6 \mathrm{~Hz}, \mathrm{H} 14 \mathrm{~A}\right), 8.41$ $\left(\mathrm{d}, 2 \mathrm{H},{ }^{4} J_{\mathrm{H}-\mathrm{H}}=1.6 \mathrm{~Hz}, \mathrm{H} 14 \mathrm{~B}\right), 7.70-7.56(\mathrm{~m}, 8 \mathrm{H}, \mathrm{H} 10, \mathrm{H} 11), 4.94\left(\mathrm{~d},{ }^{3} J_{\mathrm{H}-\mathrm{H}}=9.5 \mathrm{~Hz}, \mathrm{H} 5\right), 4.88-4.81(\mathrm{~m}$, 1H, H2), 3.96 (s, 12H, H18), 3.21 (s, 1H, H4), 1.35 (s, 9H, H8), 1.19 (d, 3H, $\left.{ }^{2} J_{\mathrm{H}-\mathrm{H}}=6.3 \mathrm{~Hz}, \mathrm{H} 3\right)$.

${ }^{13} \mathrm{C}-\mathrm{NMR}\left(75 \mathrm{MHz}, \mathrm{CDCl}_{3}\right): \delta$ [ppm] = 166.21, 166.18 (4C, C17), 132.17 (br) (4C, C14), 131.16, 131.12 (2C, C13), 127.3, 127.0, 126.7, 126.3 (8C, C10, C11); 80.8 (1C, C1); 79.7 (1C, C7); 52.5 (br) (4C, C18); 28.3 (3C, C8), $24.90(1 \mathrm{C}, \mathrm{C} 3)$.

IR (ATR): $\widetilde{v}\left[\mathrm{~cm}^{-1}\right]=3425(\mathrm{br}), 2979(\mathrm{w}), 2954(\mathrm{w}), 2929(\mathrm{w}), 1722(\mathrm{~s}), 1679(\mathrm{~m}), 1602(\mathrm{w}), 1512(\mathrm{~m})$, $1448(\mathrm{~m}), 1432(\mathrm{~m}), 1363(\mathrm{~m}), 1340(\mathrm{~m}), 1238$ (s), 1161 (m), $1132(\mathrm{~m}), 1105(\mathrm{~m}), 1074(\mathrm{~m}), 1054(\mathrm{~m}), 999$ (m), $964(\mathrm{~m}), 914(\mathrm{w}), 829(\mathrm{~m}), 754(\mathrm{~s}), 721(\mathrm{~m})$.

\subsection{Di-(O,O-dimethyl-3', $5^{\prime}$-dicarboxybiphenyl)-N-tert-butyloxycarbonyl-valinol (4b)}<smiles>COC(=O)c1cc(C(=O)OC)cc(-c2ccc(C(O)(c3ccc(-c4cc(C(=O)OC)cc(C(=O)OC)c4)cc3)C(NC(=O)OC(C)(C)C)C(C)C)cc2)c1</smiles> 
The reaction was performed according to the procedure described in Section 3.6

Yield: $4.22 \mathrm{~g}$ (5.70 mmol, 64\%); yellow foam; $\mathrm{M}:\left[\mathrm{C}_{42} \mathrm{H}_{45} \mathrm{NO}_{11}\right] 739.8 \frac{\mathrm{g}}{\mathrm{mol}} ; \mathrm{TLC}: R_{f}=0.25$

(heptane/EtOAc, 2:1); opt. rot.: $[\alpha]_{\mathrm{D}}^{27}=-12.8(\mathrm{c}=0.51, \mathrm{MeOH})$; HRMS (ESI+): $\frac{m}{z}=762.2894$ (calc. for $\mathrm{NaM}^{+}$: 762.2890), elem. anal.: \% $=\mathrm{C}(67.67), \mathrm{H}(6.34), \mathrm{N}(1.50), \mathrm{O}(22.39)$, calc. for $\mathrm{C}_{42} \mathrm{H}_{45} \mathrm{NO}_{11}$ : C (68.19), H (6.13), N (1.89), O (23.79) .

${ }^{1} \mathrm{H}-\mathrm{NMR}\left(300 \mathrm{MHz}, \mathrm{CDCl}_{3}\right): \delta[\mathrm{ppm}]=8.60\left(\mathrm{t}, 2 \mathrm{H},{ }^{4} J_{\mathrm{H}-\mathrm{H}}=1.6 \mathrm{~Hz}, \mathrm{H} 17\right), 8.59-8.57(\mathrm{~m}, 4 \mathrm{H}, \mathrm{H} 15)$, 7.74-7.68 (m, 2H, H11 or H12), 7.76-7.59 (m, 4H, H11 or H12), 7.58-7.52 (m, 2H, H11 or H12), $5.27(\mathrm{~d}, 1 \mathrm{H}$, $\left.{ }^{3} J_{\mathrm{H}-\mathrm{H}}=10.3 \mathrm{~Hz}, \mathrm{H6}\right), 4.77-4.65(\mathrm{~m}, 1 \mathrm{H}, \mathrm{H} 2), 3.93(\mathrm{~s}, 6 \mathrm{H}, \mathrm{H} 19 \mathrm{~A}), 3.92(\mathrm{~s}, 6 \mathrm{H}, \mathrm{H} 19 \mathrm{~B}), 2.00-1.86(\mathrm{~m}, 1 \mathrm{H}, \mathrm{H} 3)$, $1.30(\mathrm{~s}, 9 \mathrm{H}, \mathrm{C} 9), 0.98\left(\mathrm{t}, 3 \mathrm{H},{ }^{3} \mathrm{~J}_{\mathrm{H}-\mathrm{H}}=6.6 \mathrm{~Hz}, \mathrm{H} 4 \mathrm{~A}\right), 0.95\left(\mathrm{t}, 3 \mathrm{H},{ }^{3} J_{\mathrm{H}-\mathrm{H}}=6.9 \mathrm{~Hz}, \mathrm{H} 4 \mathrm{~B}\right)$.

${ }^{13} \mathrm{C}-\mathrm{NMR}\left(75 \mathrm{MHz}, \mathrm{CDCl}_{3}\right): \delta$ [ppm] = $166.2(4 \mathrm{C}, \mathrm{C} 18) 156.4(1 \mathrm{C}, \mathrm{C} 7), 146.5,145.6$ (4C, C16), 141.4, 141.3, 137.4(br) (4C, C10, C13), 132.14, 132.09 (4C, C15), 131.08, 131.05 (2C, C14), 129.31, 129.22 (2C, C17), 127.19, 126.94, 126.59, 126.10 (8C, C11, C12), 82.3 (1C, C1), 79.3 (1C, C8), 59.4 (1C, C2), 52.45, 52.42 (4C, C19), 28.9 (1C, C2), 28.3 (3C, C9), 22.8, 17.7 (2C, C4);

IR (KBr): $\widetilde{v}\left[\mathrm{~cm}^{-1}\right]=2954(\mathrm{w}), 2873(\mathrm{w}), 2848(\mathrm{w}), 1726(\mathrm{~s}), 1596(\mathrm{w}), 1434(\mathrm{~m}), 1367(\mathrm{w}), 1342(\mathrm{~m})$, $1236(\mathrm{~s}), 1164(\mathrm{~m}), 1132(\mathrm{~m}), 1000(\mathrm{~m}), 914(\mathrm{~m}), 873(\mathrm{~m}), 798(\mathrm{~m}), 754(\mathrm{~m}), 719(\mathrm{~m})$.

\subsection{Di-(O,O-dimethyl-3',5'-dicarboxybiphenyl)-N-tert-butyloxycarbonyl-leucinol (4c)}

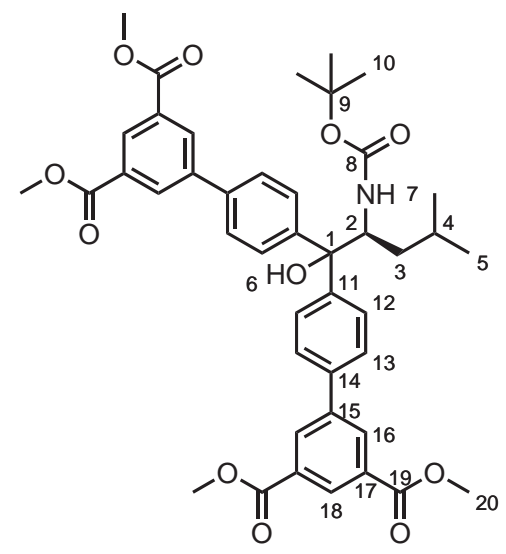

The reaction was performed according to the procedure described in Section 3.6 .

Yield: $3.77 \mathrm{~g}$ (5.00 mmol, 50\%); orange foam; M: $\left[\mathrm{C}_{43} \mathrm{H}_{47} \mathrm{NO}_{11}\right] 753.8 \frac{\mathrm{g}}{\mathrm{mol}} ;$ TLC: $R_{f}=0.22$

(heptane/EtOAc, 2:1); opt. rot.: $[\alpha]_{\mathrm{D}}^{27}=+8.0(\mathrm{c}=0.80, \mathrm{MeOH})$; HRMS (ESI+): $\frac{m}{z}=776.3050$ (calc. for $\mathrm{NaH}^{+}$: 776.3047); elem. anal.: \% $=\mathrm{C}(69.94), \mathrm{H}(6.56), \mathrm{N}(1.45), \mathrm{O}(22.85)$, calc. for $\mathrm{C}_{43} \mathrm{H}_{47} \mathrm{NO}_{11}$ : C (68.51), H (6.28), N (1.86), O (23.35) .

${ }^{1} \mathrm{H}-\mathrm{NMR}\left(300 \mathrm{MHz}, \mathrm{CDCl}_{3}\right): \delta[\mathrm{ppm}]=8.63\left(\mathrm{t}, 1 \mathrm{H},{ }^{4} J_{\mathrm{H}-\mathrm{H}}=1.6 \mathrm{~Hz}, \mathrm{H} 18 \mathrm{~A}\right), 8.61\left(\mathrm{t}, 1 \mathrm{H},{ }^{4} J_{\mathrm{H}-\mathrm{H}}=1.6\right.$ $\mathrm{Hz}, \mathrm{H} 18 \mathrm{~B}), 8.43\left(\mathrm{~d}, 1 \mathrm{H},{ }^{4} J_{\mathrm{H}-\mathrm{H}}=1.6 \mathrm{~Hz}, \mathrm{H} 16 \mathrm{~A}\right), 8.38\left(\mathrm{~d}, 1 \mathrm{H},{ }^{4} J_{\mathrm{H}-\mathrm{H}}=1.6 \mathrm{~Hz}, \mathrm{H} 16 \mathrm{~B}\right), 7.74-7.53(\mathrm{~m}, 8 \mathrm{H}$, $\mathrm{H} 12, \mathrm{H} 13), 4.86\left(\mathrm{~d}, 1 \mathrm{H},{ }^{3} J_{\mathrm{H}-\mathrm{H}}=9.8 \mathrm{~Hz}, \mathrm{H7}\right), 4.83-4.71(\mathrm{~m}, 1 \mathrm{H}, \mathrm{H} 2), 3.96(\mathrm{~s}, 6 \mathrm{H}, \mathrm{H} 20 \mathrm{~A}), 3.95(\mathrm{~s}, 6 \mathrm{H}, \mathrm{H} 20 \mathrm{~B})$, 1.88-1.65 (m, 1H, H4), 1.60-1.40 (m, 1H, H3A), 1.28 (s, 9H, H10), 1.27-1.23 (m, 1H, H3B), 1.02 (d, 3H, $\left.{ }^{3} J_{\mathrm{H}-\mathrm{H}}=6.5 \mathrm{~Hz}, \mathrm{H} 5 \mathrm{~A}\right), 0.89\left(\mathrm{~d}, 3 \mathrm{H},{ }^{3} J_{\mathrm{H}-\mathrm{H}}=6.7 \mathrm{~Hz}, \mathrm{H} 5 \mathrm{~B}\right)$.

${ }^{13} \mathrm{C}-\mathrm{NMR}\left(75 \mathrm{MHz}, \mathrm{CDCl}_{3}\right): \delta[\mathrm{ppm}]=166.21,166.19(4 \mathrm{C}, \mathrm{C} 19), 156.25(1 \mathrm{C}, \mathrm{C} 8), 145.42,145.05$ (4C, C17), 141.52, 141.22, 137.64, 137.47 (4C, C11, C14), 132.1, (4C, C16) 131.1, 131.1 (2C, C15), 129.37, 129.22 (2C, C18), 127.2, 126.9, 126.7, 126.3 (8C, C12, C13), 81.37 (1C, C1), 79.41 (1C, C9), 55.08 (1C, C2), 52.46, 52.43 (4C, C20), 39.44 (1C, C3), 28.22 (3C, C10), 25.03 (1C, C4), 23.95, 22.68 (2C, C5).

IR (ATR): $\widetilde{v}\left[\mathrm{~cm}^{-1}\right]=2952(\mathrm{w}), 2929(\mathrm{w}), 2867(\mathrm{w}), 1726(\mathrm{~s}), 1598(\mathrm{w}), 1432(\mathrm{~m}), 1342(\mathrm{~m}), 1240(\mathrm{~s}), 1161$ (m), $1072(\mathrm{~m}), 1000(\mathrm{~m}), 829(\mathrm{~m}), 754(\mathrm{~s}), 721(\mathrm{~m})$. 


\subsection{Di-(O,O-dimethyl-3', $5^{\prime}$-dicarboxybiphenyl)- $N$-benzyl-prolinol (4d)}

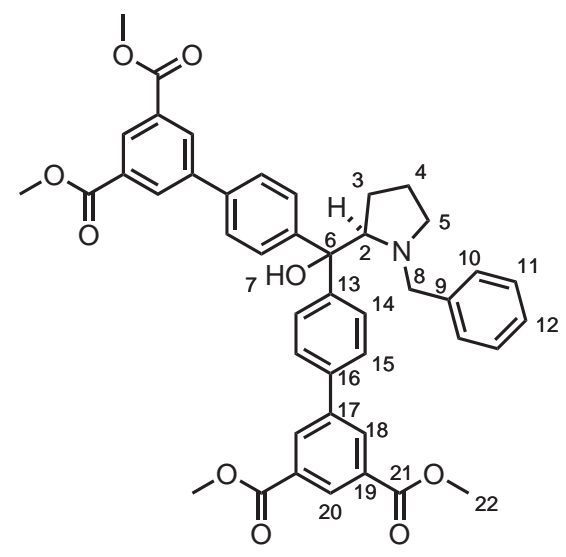

The reaction was performed according to the procedure described in Section 3.6 Yield: $4.03 \mathrm{~g}$ (5.54 mmol, 59\%); yellow foam; $\mathbf{M}:\left[\mathrm{C}_{44} \mathrm{H}_{41} \mathrm{NO}_{9}\right] 727.8 \frac{\mathrm{g}}{\mathrm{mol}} ; \mathrm{TLC}: R_{f}=0.67$ (heptane/EtOAc, 2:1); opt. rot.: $[\alpha]_{\mathrm{D}}^{26}=+54.0\left(\mathrm{c}=0.80\right.$, MeOH); HRMS (ESI+): $\frac{m}{z}=728.2913$ (calc. for $\mathrm{MH}^{+}:$728.2854).

${ }^{1} \mathrm{H}-\mathrm{NMR}\left(300 \mathrm{MHz}, \mathrm{CDCl}_{3}\right): \delta[\mathrm{ppm}]=8.62\left(\mathrm{t}, 1 \mathrm{H},{ }^{4} J_{\mathrm{H}-\mathrm{H}}=1.6 \mathrm{~Hz}, \mathrm{H} 20 \mathrm{~A}\right), 8.59\left(\mathrm{t}, 1 \mathrm{H},{ }^{4} J_{\mathrm{H}-\mathrm{H}}=1.6\right.$ $\mathrm{Hz}, \mathrm{H} 20 \mathrm{~B}), 8.43\left(\mathrm{~d}, 2 \mathrm{H},{ }^{4} J_{\mathrm{H}-\mathrm{H}}=1.6 \mathrm{~Hz}, \mathrm{H} 18 \mathrm{~A}\right), 8.38\left(\mathrm{~d}, 2 \mathrm{H},{ }^{4} J_{\mathrm{H}-\mathrm{H}}=1.6 \mathrm{~Hz}, \mathrm{H} 18 \mathrm{~B}\right), 7.92-7.85(\mathrm{~m}, 2 \mathrm{H}$, H14A or H15A), 7.76-7.70 (m, 2H, H14B or H15B), 7.66-7.57 (m, 4H, H14 or H15), 7.29-7.18 (m, 3, H10, $\mathrm{H} 12), 7.12-7.05(\mathrm{~m}, 2 \mathrm{H}, \mathrm{H} 13), 4.10\left(\mathrm{dd}, 1 \mathrm{H},{ }^{3} \mathrm{~J}_{\mathrm{H}-\mathrm{H}}=9.4,4.7 \mathrm{~Hz}, \mathrm{H} 2\right) 3.96(\mathrm{~s}, 6 \mathrm{H}, \mathrm{C} 22 \mathrm{~A}), 3.94(\mathrm{~s}, 6 \mathrm{H}, \mathrm{C} 22 \mathrm{~B})$, $3.41\left(\mathrm{~d}, 1 \mathrm{H},{ }^{2} J_{\mathrm{H}-\mathrm{H}}=12.6 \mathrm{~Hz}, \mathrm{C} 8 \mathrm{~A}\right), 3.13\left(\mathrm{~d}, 1 \mathrm{H},{ }^{2} J_{\mathrm{H}-\mathrm{H}}=12.6 \mathrm{~Hz}, \mathrm{C} 8 \mathrm{~B}\right), 2.98\left(\mathrm{dd}, 1 \mathrm{H},{ }^{3} J_{\mathrm{H}-\mathrm{H}}=9.7,5.1 \mathrm{~Hz}\right.$, $\mathrm{H} 5 \mathrm{~A}), 2.50-2.36(\mathrm{~m}, 1 \mathrm{H}, \mathrm{H} 5 \mathrm{~B}), 2.03\left(\mathrm{dd},{ }^{2} J_{\mathrm{H}-\mathrm{H}}=12.6 \mathrm{~Hz},{ }^{3} J_{\mathrm{H}-\mathrm{H}}=9.0 \mathrm{~Hz}, \mathrm{H} 3 \mathrm{~A}\right), 1.88-1.74(\mathrm{~m}, 1 \mathrm{H}, \mathrm{H} 3 \mathrm{~B})$, $1.74-1.55(\mathrm{~m}, 2 \mathrm{H}, \mathrm{H} 4)$.

${ }^{13} \mathrm{C}-\mathrm{NMR}\left(75 \mathrm{MHz}, \mathrm{CDCl}_{3}\right): \delta$ [ppm] = 166.24, $166.20(4 \mathrm{C}, \mathrm{C} 22), 148.16,146.72(4 \mathrm{C}, \mathrm{C} 19), 141.56$, 141.33 (2C, C13 or C16), 139.44 (1C, C9), 137.13, 137.03 (4C, C13 or C16) 132.16, 132.13 (4C, C18), 131.08, 131.03 (2C, C17), 129.20 (br) (2C, C20), 128.51, 128.22 (4C, C10, C11), 127.10, 127.03 (4C, C14 or C15), 126.97 (1C, C12) 126.32, 126.16 (4C, C14 or C15);

IR (KBr): $\widetilde{v}\left[\mathrm{~cm}^{-1}\right]$ = $3251(\mathrm{br}), 3135(\mathrm{w}), 2962(\mathrm{w}), 2933(\mathrm{w}), 2873(\mathrm{w}), 1747(\mathrm{~s}), 1587(\mathrm{w}), 1485(\mathrm{~m})$, $1398(\mathrm{~m}), 1365(\mathrm{~m}), 1313(\mathrm{~m}), 1240(\mathrm{~m}), 1074(\mathrm{~m}), 1041(\mathrm{~m}), 995(\mathrm{~m}), 945(\mathrm{~m}), 912(\mathrm{~m}), 810(\mathrm{~m})$.

\subsection{1,1-Di-(3',5'-dicarboxybiphenyl)- $N$-tert-butyloxycarbonyl-alaninol (5a)}<smiles>CCCC(=O)N[C@@H](C)C(O)(c1ccc(-c2cc(C(=O)O)cc(C(=O)O)c2)cc1)c1ccc(-c2cc(C(=O)O)cc(C(=O)O)c2)cc1</smiles>

To a solution of the tetramethyl ester $4 \mathrm{a}(2.00 \mathrm{~g}, 2.81 \mathrm{mmol}, 1 \mathrm{eq}$.$) in tetrahydrofuran (THF) (100 \mathrm{~mL})$ water $(100 \mathrm{~mL})$ and a solution of potassium hydroxide $\left(\mathrm{KOH}, 2 \frac{\mathrm{mol}}{\mathrm{L}}, 9.8 \mathrm{~mL}, 20 \mathrm{mmol}, 7\right.$ eq.) was added. The reaction solution was heated to reflux for 2-3 hours and was monitored by TLC. Upon consumption of compound $4 \mathbf{a}$, the reaction mixture was allowed to cool to room temperature. The reaction mixture was concentrated in vacuum to remove most of the THF. The residue was diluted with water $(200 \mathrm{~mL})$ and concentrated hydrochloric acid was added dropwise until $\mathrm{pH} 2-5$ was reached. This resulted in the precipitation of the tetracarboxylic acid. The suspension was centrifuged and the liquid phase was discarded. The precipitate was washed with water and then dried in vacuum. 
Yield: $1.57 \mathrm{~g}$ (2.40 mmol, $85 \%)$; white powder; $\mathrm{M}:\left[\mathrm{C}_{36} \mathrm{H}_{33} \mathrm{NO}_{11}\right] 655.6 \frac{\mathrm{g}}{\mathrm{mol}}$; opt. rot.: $[\alpha]_{\mathrm{D}}^{27}=-21.7$ (c = 0.24, MeOH); HRMS (ESI+): $\frac{m}{z}=678.1960$ (calc. for $\mathrm{MNa}^{+}: 655.1951$ ), elem. anal.: $\%=\mathrm{C}(59.47)$, $\mathrm{H}(5.54), \mathrm{N}(1.63), \mathrm{O}(26.29)$, calc. for $\mathrm{C}_{36} \mathrm{H}_{33} \mathrm{NO}_{11}$ : C (65.95), $\mathrm{H}(5.07), \mathrm{N}(2.14), \mathrm{O}$ (26.84) .

${ }^{1} \mathrm{H}-\mathrm{NMR}\left(300 \mathrm{MHz}, \mathrm{CD}_{3} \mathrm{OD}\right): \delta[\mathrm{ppm}]=8.60-8.58(\mathrm{~m}, 2 \mathrm{H}, \mathrm{H} 16), 8.37\left(\mathrm{~d}, 2 \mathrm{H}^{4} J_{\mathrm{H}-\mathrm{H}}=1.7 \mathrm{~Hz}, \mathrm{H} 14 \mathrm{~A}\right)$, $8.36\left(\mathrm{~d}, 2 \mathrm{H},{ }^{4} J_{\mathrm{H}-\mathrm{H}}=1.6 \mathrm{~Hz}, \mathrm{H} 14 \mathrm{~B}\right), 7.70-7.54(\mathrm{~m}, 8 \mathrm{H}, \mathrm{H} 10, \mathrm{H} 11), 4.84\left(\mathrm{q}, 1 \mathrm{H}^{3} J_{\mathrm{H}-\mathrm{H}}=6.5 \mathrm{~Hz}, \mathrm{H} 2\right), 1.30(\mathrm{~s}$, $9 \mathrm{H}, \mathrm{H} 8), 1.12\left(\mathrm{~d}, 3 \mathrm{H},{ }^{3} J_{\mathrm{H}-\mathrm{H}}=6.5 \mathrm{~Hz}, \mathrm{H} 3\right)$.

${ }^{13} \mathrm{C}-N M R\left(75 \mathrm{MHz}, \mathrm{CD}_{3} \mathrm{OD}\right): \delta$ [ppm] = 168.84 (br) (4C, C14), 157.67 (1C, C6), 147.63, 146.93 (2C, C9 or C12), 142.69, 142.51 (2C, C9 or C12), 138.60 (2C, C13), 133.10 (br) (4C, C14), 132.89 (br) (4C, C17), 130.48, 130.44 (2C, C16), 128.05, 127.81, 127.65, 127.58 (8C, C10, C11), 81.38 (1C, C1), 80.18 (1C, C7), 53.42 (1C, C2), 28.68 (3C, C8), 26.44 (1C, C3);

IR (ATR): $\widetilde{v}\left[\mathrm{~cm}^{-1}\right]$ ] = $3425(\mathrm{br}), 2977(\mathrm{w}), 2952(\mathrm{w}), 2931(\mathrm{w}), 2561(\mathrm{br}), 1712(\mathrm{~s}), 1695(\mathrm{~s}), 1600(\mathrm{~m})$, $1513(\mathrm{~m}), 1446(\mathrm{~m}), 1392(\mathrm{~m}), 1365(\mathrm{~m}), 1338(\mathrm{~m}), 1240(\mathrm{br}), 1162$ (s), 1107 (m), $1056(\mathrm{~m}), 1000(\mathrm{w}), 916$ $(\mathrm{m}), 829(\mathrm{~m}), 757(\mathrm{~s}), 719(\mathrm{~m}), 671(\mathrm{~m})$.

\subsection{1,1-Di-(3',5'-dicarboxybiphenyl)- $N$-tert-butyloxycarbonyl-valinol (5b)}<smiles>CC(C)C(NC(=O)OC(C)(C)C)C(O)(c1ccc(-c2cc(C(=O)O)cc(C(=O)O)c2)cc1)c1ccc(-c2cc(C(=O)O)cc(C(=O)O)c2)cc1</smiles>

The reaction was performed according to the procedure described in Section 3.10 Yield: $1.47 \mathrm{~g}(2.15 \mathrm{mmol}, 80 \%) ; \mathrm{M}:\left[\mathrm{C}_{38} \mathrm{H}_{37} \mathrm{NO}_{11}\right] 683.2 \frac{\mathrm{g}}{\mathrm{mol}}$; opt. $\operatorname{rot} .:[\alpha]_{\mathrm{D}}^{27}=-12.7(\mathrm{c}=0.22, \mathrm{MeOH})$; HRMS (ESI+): $\frac{m}{z}=706.2183$ (calc. for $\mathrm{NaM}^{+}:$706.2264) elem. anal.: $\%=\mathrm{C}(61.06), \mathrm{H}(5.31), \mathrm{N}(1.68), \mathrm{O}$ (24.84), calc. for $\mathrm{C}_{39} \mathrm{H}_{39} \mathrm{NO}_{11}$ : C (66.76), $\mathrm{H}(5.45), \mathrm{N}(2.05), \mathrm{O}(25.74)$.

${ }^{1} \mathrm{H}-\mathrm{NMR}\left(300 \mathrm{MHz}, \mathrm{CD}_{3} \mathrm{OD}\right): \delta[\mathrm{ppm}]=8.63-8.58(\mathrm{~m}, 2 \mathrm{H}, \mathrm{H} 17), 8.45\left(\mathrm{~d}, 2 \mathrm{H},{ }^{3} J_{\mathrm{H}-\mathrm{H}}=1.6 \mathrm{~Hz} \mathrm{H} 15 \mathrm{~A}\right)$, $8.40\left(\mathrm{~d},{ }^{3} J_{\mathrm{H}-\mathrm{H}}=1.6 \mathrm{~Hz} \mathrm{H} 15 \mathrm{~B}\right), 7.79-7.57(\mathrm{~m}, 8 \mathrm{H}, \mathrm{H} 11, \mathrm{H} 12), 6.26\left(\mathrm{~d}, 1 \mathrm{H},{ }^{3} J_{\mathrm{H}-\mathrm{H}}=10.5 \mathrm{~Hz}, \mathrm{H6}\right), 4.68(\mathrm{dd}$, $\left.1 \mathrm{H},{ }^{3} J_{\mathrm{H}-\mathrm{H}}=10.5,2.2 \mathrm{~Hz}, \mathrm{H} 2\right), 1.98-1.82(\mathrm{~m}, 1 \mathrm{H}, \mathrm{H} 3), 1.34\left(\mathrm{~s}, 9 \mathrm{H}, \mathrm{H} 9,1.00\left(\mathrm{~d}, 3 \mathrm{H},{ }^{3} J_{\mathrm{H}-\mathrm{H}}=6.7 \mathrm{~Hz}, \mathrm{H} 4 \mathrm{~A}\right)\right.$, $0.90\left(\mathrm{~d}, 3 \mathrm{H},{ }^{3} J_{\mathrm{H}-\mathrm{H}}=6.7 \mathrm{~Hz}, \mathrm{H} 4 \mathrm{~B}\right)$;

${ }^{13}$ C-NMR (75 MHz, DMSO- $\left.\mathbf{d}_{6}\right): \delta$ [ppm] = $167.0(4 \mathrm{C}, \mathrm{C} 18), 156.4(1 \mathrm{C}, \mathrm{C} 7), 148.3,146.5(2 \mathrm{C}, \mathrm{C} 10$ or C13), 141.06, 140.95 (2C, C10 or C13), 137.0 (2C, C14), 132.8, 132.7 (4C, C16), 131.41, 131.35 (4C, C15), 129.16 (2C, C17), 127.14, 127.07, 126.8, 126.4 (8C, C11, C12), 81.6 (1C, C1), 78.4 (1C, C8), 60.1 (1C, C2) 28.9 (1C, C3) 28.6 (3C, C9), 23.5 (1C, C4A), 18.5 (1C, C4B);

IR (ATR): $\widetilde{v}\left[\mathrm{~cm}^{-1}\right]=3415(\mathrm{br}), 2970,(\mathrm{w}), 2931(\mathrm{w}), 2873(\mathrm{w}), 2514(\mathrm{br}), 1693(\mathrm{~s}), 1600(\mathrm{~m}), 1508(\mathrm{~m})$, $1448(\mathrm{~m}), 1394(\mathrm{~m}), 1367(\mathrm{~m}), 1238(\mathrm{~s}), 1161(\mathrm{~m}), 914(\mathrm{~m}), 827(\mathrm{~m}), 759(\mathrm{~m}), 663(\mathrm{~m})$. 


\subsection{1,1-Di-(3',5'-dicarboxybiphenyl)- $N$-tert-butyloxycarbonyl-leucinol (5c)}<smiles>CC(C)C[C@H](NC(=O)OC(C)(C)C)C(O)(c1ccc(-c2cc(C(=O)O)cc(C(=O)O)c2)cc1)c1ccc(-c2cc(C(=O)O)cc(C(=O)O)c2)cc1</smiles>

The reaction was performed according to the procedure described in Section 3.10 .

Yield: $1.12 \mathrm{~g}(1.64 \mathrm{mmol}, 82 \%)$; opt. rot.: $[\alpha]_{\mathrm{D}}^{26}=+13.6(\mathrm{c}=0.44, \mathrm{MeOH})$; HRMS (ESI+): 720.2431 (calc. for $\mathrm{NaM}^{+}$: 720.2421) elem. anal.: \% = C (54.52), $\mathrm{H}(5.06), \mathrm{N}(1.42), \mathrm{O}(24.51)$, calc. for $\mathrm{C}_{39} \mathrm{H}_{39} \mathrm{NO}_{11}$ : C (67.13), H (5.63), N (2.01), O (25.22) .

${ }^{1} \mathbf{H}-N M R\left(300 \mathrm{MHz}\right.$, DMSO-d $\left.{ }_{6}\right): \delta[\mathrm{ppm}]=13.38(\mathrm{~s}, 4 \mathrm{H}, \mathrm{H} 20), 8.43\left(\mathrm{t}, 2 \mathrm{H},{ }^{4} J_{\mathrm{H}-\mathrm{H}}=1.5 \mathrm{~Hz}, \mathrm{H} 18\right)$, $8.36\left(\mathrm{~d}, 2 \mathrm{H},{ }^{3} J_{\mathrm{H}-\mathrm{H}}=1.5 \mathrm{~Hz}, \mathrm{H} 16 \mathrm{~A}\right), 8.30\left(\mathrm{~d}, 2 \mathrm{H},{ }^{3} J_{\mathrm{H}-\mathrm{H}}=1.5 \mathrm{~Hz}, \mathrm{H} 16 \mathrm{~B}\right), 7.76-7.60(\mathrm{~m}, 8 \mathrm{H}, \mathrm{H} 12, \mathrm{H} 13), 6.43$ $\left(\mathrm{d}, 1 \mathrm{H},{ }^{3} J_{\mathrm{H}-\mathrm{H}}=10.0 \mathrm{~Hz}, \mathrm{H} 7\right), 5.71(\mathrm{~s}, 1 \mathrm{H}, \mathrm{H} 6), 4.78\left(\mathrm{t}, 1 \mathrm{H},{ }^{3} J_{\mathrm{H}-\mathrm{H}}=10.6 \mathrm{~Hz}, \mathrm{H} 2\right), 1.63-1.44(\mathrm{~m}, 2 \mathrm{H}, \mathrm{H} 3)$, $1.27(\mathrm{~s}, 9 \mathrm{H}, \mathrm{H} 10), 1.01-0.90(\mathrm{~m}, 1 \mathrm{H}, \mathrm{H} 4), 0.88\left(\mathrm{~d}, 3 \mathrm{H},{ }^{3} J_{\mathrm{H}-\mathrm{H}}=6.4 \mathrm{~Hz}, \mathrm{H} 5 \mathrm{~A}\right), 0.80\left(\mathrm{~d}, 3 \mathrm{H},{ }^{3} J_{\mathrm{H}-\mathrm{H}}=6.4 \mathrm{~Hz}\right.$, H5B).

${ }^{13} \mathrm{C}-\mathrm{NMR}\left(75 \mathrm{MHz}, \mathrm{DMSO}-\mathbf{d}_{6}\right): \delta$ [ppm] = $166.5(4 \mathrm{C}, \mathrm{C} 19), 155.7$ (1C, C8), 147.3, 146.0 (2C, C11 or C14), 140.9, 140.6 (2C, C11 or C14), 136.4, 136.1 (2C, C15) 132.1 (4C, C17), 131.1 (br) (4C, C16), 128.7 (br) (2C, C18), 126.7 (br), 126.29, 126.04 (8C, C12, C13), 80.4 (1C, C1), 77.8 (1C, C9), 54.4 (1C, C2), 39.8 (1C, C3, determined from HMBC) 28.1 (3C, C10), 24.0 (1C, C5A), 21.4 (1C, C4), 21.5 (1C, C5B);

IR (ATR): $\widetilde{v}\left[\mathrm{~cm}^{-1}\right]=3415(\mathrm{w}), 2966(\mathrm{w}), 2865(\mathrm{w}), 2528(\mathrm{br}), 1967(\mathrm{~s}), 1670(\mathrm{~s}), 1602(\mathrm{~m}), 1521(\mathrm{~m})$, $1450(\mathrm{~m}), 1365(\mathrm{~m}), 1272(\mathrm{~m}), 1249(\mathrm{~m}), 1201(\mathrm{~m}), 1172(\mathrm{~m}), 916(\mathrm{~m}), 825(\mathrm{~m}), 759(\mathrm{~m}), 684(\mathrm{~m}), 669(\mathrm{~m})$.

\subsection{1,1-Di-(3', 5'-dicarboxybiphenyl)-alaninol (6a)}<smiles>C[C@H](N)C(O)(c1ccc(-c2ccc(C(=O)O)cc2)cc1)c1ccc(-c2cc(C(=O)O)cc(C(=O)O)c2)cc1</smiles>

A suspension of tetracarboxylic acid $5 \mathbf{a}\left(600 \mathrm{mg}, 0.92 \mathrm{mmol}, 1\right.$ eq.) in $\mathrm{CH}_{2} \mathrm{Cl}_{2}(100 \mathrm{~mL})$ was cooled to $0^{\circ} \mathrm{C}$. Trifluoro acetic acid $(25 \mathrm{~mL})$ was added. The reaction solution was stirred for two hours while the mixture was allowed to warm to room temperature. Upon the addition of diethyl ether $(150 \mathrm{~mL}) \mathrm{a}$ white precipitate of the ammonium salt of the tetracarboxylic acid was obtained. The suspension was cooled to $4{ }^{\circ} \mathrm{C}$ and the precipitate was collected by filtration. After washing with diethyl ether $(50 \mathrm{~mL})$, the ammonium salt of the tetracarboxylic acid was dried in vacuum.

Yield: $458 \mathrm{mg}$ (0.68 mmol, 75\%); white powder; $\mathbf{M}:\left[\mathrm{C}_{33} \mathrm{H}_{26} \mathrm{~F}_{3} \mathrm{NO}_{11}\right] 669.9 \frac{\mathrm{g}}{\mathrm{mol}}$; opt. rot.: $[\alpha]_{\mathrm{D}}^{27}=-17.5$ $\left(\mathrm{c}=0.40, \mathrm{MeOH}\right.$ ); HRMS (ESI+): $\frac{m}{z}=556.1611$ (calc. for $\mathrm{C}_{31} \mathrm{H}_{26} \mathrm{NO}_{11}{ }^{+}: 556.1602$ ) elem. anal.: $\%=\mathrm{C}$ (54.52), $\mathrm{H}$ (5.06), $\mathrm{F}$ (n.d.), $\mathrm{N}(1.40), \mathrm{O}$ (24.51) calc. for $\mathrm{C}_{23} \mathrm{H}_{29} \mathrm{Br}_{2} \mathrm{NO}_{3}$ : C (60.76), $\mathrm{H}(4.53), \mathrm{F}(8.01), \mathrm{N}$ (1.97), O (24.73). 
${ }^{1}$ H-NMR (300 MHz, CD ${ }_{3}$ OD): $\delta$ [ppm] = 8.63-8.56 (m, 2H, H13), 8.44-8.40 (m, 2H, H11), 7.81-7.65 $(\mathrm{m}, 8 \mathrm{H}, \mathrm{H} 7, \mathrm{H} 8), 4.60\left(\mathrm{q}, 1 \mathrm{H},{ }^{3} \mathrm{~J}_{\mathrm{H}-\mathrm{H}}=6.5 \mathrm{~Hz} \mathrm{H} 2\right), 1.41\left(\mathrm{~d}, 3 \mathrm{H},{ }^{3} \mathrm{~J}_{\mathrm{H}-\mathrm{H}}=6.5 \mathrm{~Hz}, \mathrm{H} 3\right)$.

${ }^{13} \mathrm{C}-\mathrm{NMR}\left(75 \mathrm{MHz}, \mathrm{CD}_{3} \mathrm{OD}\right): \delta[\mathrm{ppm}]=168.7$ (br) $(4 \mathrm{C}, \mathrm{C} 14), 144.73,144.58$ (4C, C6 or C9), 142.34, 142.28 (4C, C6 or C9), 139.82, 139.61 (2C, C10), 133.23, 133.20 (4C, C14), 132.99, 132.96 (4C, C11), 130.7 (br) (2C, C13), 128.56, 128.18, 128.07, 127.87 (8C, C7, C8), 78.95 (1C, C1), 55.02 (1C, C2), 14.75 (1C, C3);

IR (ATR): $\widetilde{v}\left[\mathrm{~cm}^{-1}\right]=3417(\mathrm{w}), 2977(\mathrm{~m}), 2954(\mathrm{~m}), 2931(\mathrm{~m}), 1695(\mathrm{~s}), 1600(\mathrm{~m}), 1510(\mathrm{~m}), 1448(\mathrm{~m})$, $1392(\mathrm{~m}), 1367(\mathrm{~m}), 1340(\mathrm{~m}), 1240(\mathrm{~s}), 1161(\mathrm{~s}), 1058(\mathrm{~m}), 1000(\mathrm{~m}), 912(\mathrm{~m}), 827(\mathrm{~s}), 757(\mathrm{~s})$.

\subsection{1,1-Di-(3',5'-dicarboxybiphenyl)-valinol (6b)}<smiles>CC(C)C(N)C(O)(c1ccc(-c2cc(C(=O)O)cc(C(=O)O)c2)cc1)c1ccc(-c2cc(C(=O)O)cc(C(=O)O)c2)cc1</smiles>

The reaction was performed according to the procedure described in Section 3.13

Yield: $370 \mathrm{mg}(0.53 \mathrm{mmol}, 75 \%)$; white powder; $\mathbf{M}:\left[\mathrm{C}_{35} \mathrm{H}_{30} \mathrm{~F}_{3} \mathrm{NO}_{11}\right] 697.6 \frac{\mathrm{g}}{\mathrm{mol}}$; opt. rot.: $[\alpha]_{\mathrm{D}}^{27}=-19.2$ $(\mathrm{c}=0.26, \mathrm{MeOH}) ;$ HRMS (ESI+): $\frac{m}{z}=584.1911$ (calc. for $\mathrm{C}_{33} \mathrm{H}_{30} \mathrm{NO}_{9}{ }^{+}: 584.1921$ ).

${ }^{1}$ H-NMR (300 MHz, DMSO- $\left.\mathbf{d}_{6}\right): \delta$ [ppm] = 8.60-8.58 (m, 2H, H14) 8.44-8.42 (m, 4Hm H12), 7.81$7.69(\mathrm{~m}, 8 \mathrm{H}, \mathrm{H} 8, \mathrm{H} 9), 4.58\left(\mathrm{~d},{ }^{3} J_{\mathrm{H}-\mathrm{H}}=2.8 \mathrm{~Hz}, \mathrm{H} 2\right), 2.32-2.17(\mathrm{~m}, 1 \mathrm{H}, \mathrm{H} 3), 1.18\left(\mathrm{~d}, 3 \mathrm{H},{ }^{3} J_{\mathrm{H}-\mathrm{H}}=7.1 \mathrm{~Hz}\right.$, $\mathrm{H} 4 \mathrm{~A}), 1.11\left(\mathrm{~d}, 3 \mathrm{H},{ }^{3} J_{\mathrm{H}-\mathrm{H}}=6.9 \mathrm{~Hz}, \mathrm{H} 4 \mathrm{~B}\right)$.

${ }^{13} \mathrm{C}-N M R\left(75 \mathrm{MHz}, \mathrm{CD}_{3} \mathrm{OD}\right): \delta$ [ppm] = 167.2 (br) (4C, C15), 144.7, 144.6 (4C, C7 or C10), 141.0, 140.9 (4C, C7 or C10), 138.5, 138.3 (2C, C11), 131.9, 131.8 (4C, C15), 131.6, 131.5 (4C, C12), 129.3 (br) (2C, C14), 127.2, 126.8, 126.6 (br) (8C, C8, C9), 79.4 (1C, C1), 62.1 (1C, C2), 27.1 (1C, C3), 21.3, 16.0 (2C, C4).

IR (ATR): $\widetilde{v}\left[\mathrm{~cm}^{-1}\right]=2960(\mathrm{w}), 2929(\mathrm{w}), 2873(\mathrm{~m}), 1655(\mathrm{~s}), 1594(\mathrm{~s}), 1570(\mathrm{~m}), 1545(\mathrm{~m}), 1518(\mathrm{~m}), 1395$ (s), $1366(\mathrm{~m}), 1312(\mathrm{~m}), 1247(\mathrm{~m}), 1159(\mathrm{~m}), 1090(\mathrm{~m}), 1004(\mathrm{w}), 868(\mathrm{~m}), 781(\mathrm{~s})$.

\subsection{1,1-Di-(3', 5' -dicarboxybiphenyl)-leucinol (6c)}<smiles>CNC(CC(C)C)C(O)(c1ccc(-c2cc(C(=O)O)cc(C(=O)O)c2)cc1)c1ccc(-c2cc(C(=O)O)cc(C(=O)O)c2)cc1</smiles>

The reaction was performed according to the procedure described in Section 3.13 Yield: $408 \mathrm{mg}(0.57 \mathrm{mmol}, 75 \%)$; white powder; $\mathbf{M}:\left[\mathrm{C}_{36} \mathrm{H}_{32} \mathrm{~F}_{3} \mathrm{NO}_{11}\right] 711.6 \frac{\mathrm{g}}{\mathrm{mol}}$; opt. rot.: $[\alpha]_{\mathrm{D}}^{26}=+15.0$ (c = 0.60, MeOH); HRMS (ESI+): $\frac{m}{z}=598.2074$ (calc. for $\mathrm{C}_{34} \mathrm{H}_{32} \mathrm{NO}_{9}{ }^{+}$: 598.2072).

${ }^{1} \mathrm{H}-\mathrm{NMR}\left(300 \mathrm{MHz}, \mathrm{CD}_{3} \mathrm{OD}\right): \delta$ [ppm] $=8.62\left(\mathrm{q}, 2 \mathrm{H},{ }^{2} \mathrm{~J}_{\mathrm{H}-\mathrm{H}}=1.5 \mathrm{~Hz}, \mathrm{H} 15\right), 8.50-8.46(\mathrm{~m}, 4 \mathrm{H}, \mathrm{H} 13)$, 7.83-7.69 (m, 8H, H9, H10), $4.43\left(\mathrm{~d}, 1 \mathrm{H},{ }^{3} J_{\mathrm{H}-\mathrm{H}}=10.3 \mathrm{~Hz}, \mathrm{H} 2\right), 1.86-1.64(\mathrm{~m}, 2 \mathrm{H}, \mathrm{H} 3 \mathrm{~A}, \mathrm{H} 4), 1.61-1.45(\mathrm{~m}$, $1 \mathrm{H}, \mathrm{H} 3 \mathrm{~B}), 1.11\left(\mathrm{~d}, 3 \mathrm{H},{ }^{3} J_{\mathrm{H}-\mathrm{H}}=6.4 \mathrm{~Hz}, \mathrm{H} 5 \mathrm{~A}\right), 0.98\left(\mathrm{~d}, 3 \mathrm{H},{ }^{3} J_{\mathrm{H}-\mathrm{H}}=6.4 \mathrm{~Hz}, \mathrm{H} 5 \mathrm{~B}\right)$. 
${ }^{13} \mathrm{C}-\mathrm{NMR}\left(75 \mathrm{MHz}, \mathrm{CD}_{3} \mathrm{OD}\right): \delta$ [ppm] = 167.2 (4C, C16), 143.1, 142.9 (2C, C8 or C11), 141.0, 140.9 (2C, C8 or C11), 138.63(2C, C12), 131.8 (4C, C14), 131.63, 131.60 (4C, C13), 129.36 (2C, C15), 127.19, 126.89, 126.82, 126.75 (8C, C12, C13) 78.1 (1C, C1), 56.3 (1C, C2), 24.3 (1C, C3), 22.8 (1C, C4), 20.0 (br, 2C, C5).

IR (ATR): $\widetilde{v}\left[\mathrm{~cm}^{-1}\right]=3083(\mathrm{br}), 2962(\mathrm{w}), 2900(\mathrm{w}), 2871(\mathrm{w}), 1455(\mathrm{~s}), 1598(\mathrm{~m}), 1450(\mathrm{~m}), 1411(\mathrm{~m})$, $1388(\mathrm{~m}), 1319(\mathrm{~m}), 1186(\mathrm{~s}), 1141(\mathrm{~s}), 1065(\mathrm{~m}), 916(\mathrm{w}), 831(\mathrm{~m}), 761(\mathrm{~m}), 717(\mathrm{~m}), 665(\mathrm{~m})$.

\subsection{Di-(3',5'-dicarboxybiphenyl)-prolinol (6d)}

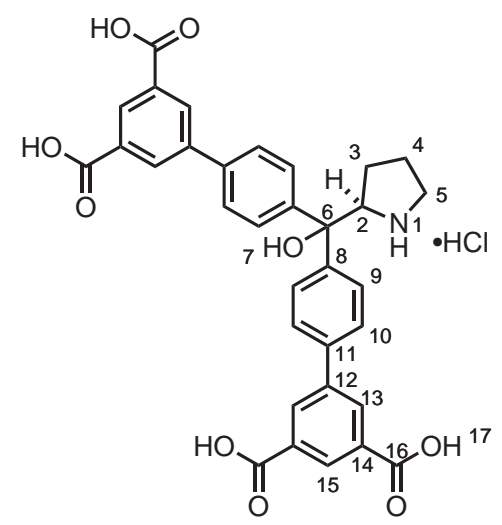

To a suspension of tetramethyl ester $4 \mathbf{d}(3.35 \mathrm{~g}$, $4.60 \mathrm{mmol}, 1 \mathrm{eq}$.$) in \mathrm{MeOH}(125 \mathrm{~mL})$ concentrated sulfuric acid $\left(\mathrm{H}_{2} \mathrm{SO}_{4}\right)$ was added dropwise until a clear, light yellow solution was obtained. This solution was transferred to a glass-lined steel autoclave, where it was stirred under nitrogen atmosphere ( $8 \mathrm{bar})$ for one hour. To this solution palladium on carbon $(\mathrm{Pd} / \mathrm{C}, 10 \mathrm{wt} \%, 400 \mathrm{mg})$ was added. The autoclave was flushed with nitrogen, then with hydrogen. The reaction solution was heated to $50^{\circ} \mathrm{C}$ and stirred under a hydrogen atmosphere (50 bar) for at least three days. The reaction solution was monitored by TLC. Upon consumption of compound $\mathbf{4 d}$, the reaction mixture was allowed to cool to room temperature and was filtered over Celite to remove the catalyst. The filter cake was washed with $\mathrm{MeOH}(50 \mathrm{~mL})$. The filtrate was concentrated in vacuum and used without further purification in the next step.

The residue was dissolved in THF $(150 \mathrm{~mL})$. Water $(150 \mathrm{~mL})$ and a solution of potassium hydroxide $\left(\mathrm{KOH}, 2 \frac{\mathrm{mol}}{\mathrm{L}}, 16.1 \mathrm{~mL}, 32.5 \mathrm{mmol}, 7 \mathrm{eq}\right.$.) were added. The reaction solution was heated to reflux for 2.5 hours. The reaction mixture was allowed to cool to room temperature and was concentrated in vacuum to remove most of the THF. The residue was diluted with water $(300 \mathrm{~mL})$ and concentrated hydrochloric acid was added dropwise until $\mathrm{pH} 2-5$ was reached. This resulted in the precipitation of the tetracarboxylic acid. The suspension was centrifuged and the liquid phase was discarded. The precipitate was washed with water and then dried in vacuum.

Yield: $2.02 \mathrm{~g}$ ( $3.29 \mathrm{mmol}, 71 \%$ over two steps); white powder; $\mathbf{M}:\left[\mathrm{C}_{33} \mathrm{H}_{28} \mathrm{ClNO}_{9}\right] 618.0 \frac{\mathrm{g}}{\mathrm{mol}}$; opt. rot.: $[\alpha]_{\mathrm{D}}^{26}=+41.3(\mathrm{c}=0.30, \mathrm{MeOH})$; HRMS (ESI+): $\frac{m}{z}=582.1773$ (calc. for $\mathrm{C}_{33} \mathrm{H}_{28} \mathrm{NO}_{9}$ : 582.1759); elem. anal.: \% $=\mathrm{C}(55.91), \mathrm{H}(4.87), \mathrm{N}(1.71), \mathrm{O}(25.11)$, calc. for $\mathrm{C}_{33} \mathrm{H}_{28} \mathrm{ClNO}_{9}: \mathrm{C}$ (61.13), $\mathrm{H}(4.57), \mathrm{Cl}(2.27), \mathrm{O}$ (23.30) .

${ }^{1}$ H-NMR (300 MHz, DMSO- $\left.\mathbf{d}_{6}\right): \delta$ [ppm] = 8.47-8.40 (m, 2H, H15), 8.37-8.29 (m, 4H, H13), 7.86-7.65 (m, 8H, H9, H10), 5.13-4.93 (m, 1H, H2), 3.28-3.09 (m, 2H, H5), 2.05-1.79 (m, 4H, H3, H4).

${ }^{13}$ C-NMR $\left(75 \mathrm{MHz}\right.$, DMSO-d $\left.\mathbf{d}_{6}\right): \delta$ [ppm] = 166.72, $166.67(4 \mathrm{C}, \mathrm{C} 16), 144.96,144.49$ (2C, C8 or C11), 140.28 (br, 2C, C8 or C11), 137.45,137.22 (2C, C12), 132.64, 132.52 (4C, C14), 131.03, 130.95 (4C, C13), 128.90 (br, 2C, C15), 126.97, 126.88, 126.79, 126.46 (8C, C9, C10), 76.97 (1C, C6), 65.04 (1C, C2), 46.69 (1C, C5), 25.97 (1C, C3), $24.11(1 \mathrm{C}, \mathrm{C} 2)$.

IR (KBr): $\widetilde{v}\left[\mathrm{~cm}^{-1}\right]$ = 3400 (br), 2966 (br), 1697 (s), 1600 (m), 1452 (m), 1388 (m), 1207 (s), 997 (m), 916 (m), $898(\mathrm{~m}), 823(\mathrm{~m}), 759(\mathrm{~s}), 665(\mathrm{~s})$. 
<smiles></smiles>

The reaction was performed according to the procedure described in Section 3.6 .

Yield: $4.90 \mathrm{~g}(7.37 \mathrm{mmol}, 75 \%)$; yellow foam; $\mathrm{M}:\left[\mathrm{C}_{38} \mathrm{H}_{35} \mathrm{NO}_{10}\right]$ 665.7 $\frac{\mathrm{g}}{\mathrm{mol}} ;$ TLC: $R_{f}=0.22$ (heptane/EtOAc, 1:1); m.p.: $118^{\circ} \mathrm{C}$; opt. rot.: $[\alpha]_{\mathrm{D}}^{27}=-71.3(\mathrm{c}=0.38, \mathrm{MeOH})$; HRMS (ESI+): $\frac{m}{z}=$ 666.2333 (calc. for $\mathrm{MH}^{+}$: 666.2333); elem. anal.: \% = C (64.09), $\mathrm{H}(5.42), \mathrm{N}(1.23), \mathrm{O}(26.03)$ calc. for $\mathrm{C}_{38} \mathrm{H}_{35} \mathrm{NO}_{10}$ : C (68.56), $\mathrm{H}(5.30), \mathrm{N}(2.10), \mathrm{O}(24.03)$.

${ }^{1}$ H-NMR (300 MHz, DMSO-- $\left.\mathbf{d}_{6}\right): \delta[\mathrm{ppm}]=8.39-8.36(\mathrm{~m}, 2 \mathrm{H}, \mathrm{H} 15), 8.33-8.28(\mathrm{~m}, 4 \mathrm{H}, \mathrm{H} 13), 8.25(\mathrm{~s}$, 1H, H1), 7.90-7.81 (m, 2H, H9A), 7.80-7.49 (m, 6H, H9B, H10), 4.61-4.53 (m, 1H, H5), 3.87 (s, 12H, H17), $1.97-1.84(\mathrm{~m}, 1 \mathrm{H}, \mathrm{H6}), 0.97\left(\mathrm{~d},{ }^{3} J_{\mathrm{H}-\mathrm{H}}=6.8 \mathrm{~Hz}, \mathrm{H} 7 \mathrm{~A}\right), 0.57\left(\mathrm{~d},{ }^{3} J_{\mathrm{H}-\mathrm{H}}=6.5 \mathrm{~Hz}, \mathrm{H} 7 \mathrm{~B}\right)$.

${ }^{13} \mathrm{C}-\mathrm{NMR}\left(75 \mathrm{MHz}, \mathrm{DMSO}-\mathrm{d}_{6}\right): \delta$ [ppm] = $165.2(4 \mathrm{C}, \mathrm{C} 16), 157.3(1 \mathrm{C}, \mathrm{C} 2), 145.1,145.4(2 \mathrm{C}, \mathrm{C} 14)$, 139.9 (br), 137.5, 136.9 (4C, C8, C11), 131.43, 131.38 (4C, C13), 128.8, 128.7 (2C, C14), 128.4 (2C, C12), 127.1, 126.8, 126.5, 126.1 (8C, C9, C10), 87.5 (1C, C4), 64.2 (1C, C5), 52.5 (4C, C17), 29.2 (1C, C6), 20.2, 14.6 $(2 \mathrm{C}, \mathrm{C} 7)$.

IR (KBr): $\widetilde{v}\left[\mathrm{~cm}^{-1}\right]=3441(\mathrm{~m}), 3360(\mathrm{~m}), 2955(\mathrm{~m}), 2878(\mathrm{w}), 2361(\mathrm{w}), 1757(\mathrm{~s}), 1728(\mathrm{~s}), 1601(\mathrm{~m}), 1514$ (w), $1448(\mathrm{~s}), 1437(\mathrm{~s}), 1375(\mathrm{~m}), 1344(\mathrm{~s}), 1246(\mathrm{~s}), 1196(\mathrm{~m}), 1134(\mathrm{~m}), 1109(\mathrm{~m}), 1072(\mathrm{~m}), 1042(\mathrm{~m}), 999$ (s), $964(\mathrm{~m}), 941(\mathrm{w}), 916(\mathrm{~m}), 854(\mathrm{~m}), 829(\mathrm{~m}), 756(\mathrm{~s}), 721(\mathrm{~m}), 702(\mathrm{~m}), 640(\mathrm{w}), 542(\mathrm{w})$.

\subsection{4,4-Di-(3',5'-dicarboxybiphenyl)-5-isopropyl-oxazolidin-2-one (8)}

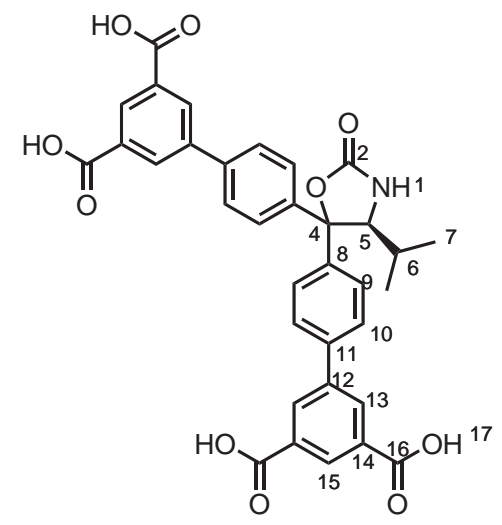

The reaction was performed according to the procedure described in Section 3.10 .

Yield: $1.57 \mathrm{~g}(2.40 \mathrm{mmol}, 85 \%)$; white powder; $\mathbf{M}:\left[\mathrm{C}_{34} \mathrm{H}_{27} \mathrm{NO}_{10}\right] 609.6 \frac{\mathrm{g}}{\mathrm{mol}} ; \mathbf{m} . \mathbf{p . :} 255^{\circ} \mathrm{C}$ (decomposition); opt. rot.: $[\alpha]_{\mathrm{D}}^{27}=-82.7\left(\mathrm{c}=0.73\right.$, MeOH); HRMS (ESI+): $\frac{m}{z}=610.1721$ (calc. for $\mathrm{MH}^{+}:$610.1708); elem. anal.: $\%=\mathrm{C}(55.79), \mathrm{H}(4.62), \mathrm{N}(1.33), \mathrm{O}(24.09)$ calc. for $\mathrm{C}_{38} \mathrm{H}_{35} \mathrm{NO}_{10}$ : C (66.99), $\mathrm{H}(4.46), \mathrm{N}(2.30), \mathrm{O}(26.25)$.

${ }^{1}$ H-NMR (300 MHz, DMSO-d ${ }_{6}$ ): $\delta$ [ppm] = 8.50-8.45 (m, 2H, H15), 8.40-8.32 (m, 4H, H13), 8.24 (s, 1H, H1), 7.89-7.82 (m, 2H, H9A), 7.80-7.73 (m, 4H; H8A, H9B), 7.70-7.63 (m, 2, H8B), 4.59-4.53 (m, 1H, $\mathrm{H} 5), 1.99-1.85(\mathrm{~m}, 1 \mathrm{H}, \mathrm{H} 6), 0.96\left(\mathrm{~d}, 3 \mathrm{H},{ }^{3} J_{\mathrm{H}-\mathrm{H}}=6.5 \mathrm{~Hz}, \mathrm{H} 6 \mathrm{~A}\right), 0.56\left(\mathrm{~d}, 3 \mathrm{H},{ }^{3} J_{\mathrm{H}-\mathrm{H}}=6.5 \mathrm{~Hz}, \mathrm{H} 6 \mathrm{~B}\right)$. 
${ }^{13}$ C-NMR (75 MHz, DMSO-d $\mathbf{d}_{6}$ ): $\delta$ [ppm] = 166.8 (4C, C16) 157.3 (1C, C2), 145.2 (2C, C14), 139.7 (2C, C10), 138.1, 137.5 (2C, C8), 132.8, 131.1 (2C, C12), 129.1 (2C, C12), 128.9, 128.7 (2C, C14), 127.2, 126.8, 126.5, 126.1 (8C, C9, C10), 87.6 (1C, C4), 64.2 (1C, C5), 29.2 (1C, C6), 20.2, 14.6 (2C, C7).

IR (KBr): $\widetilde{v}\left[\mathrm{~cm}^{-1}\right]=3454(\mathrm{~m}), 2966(\mathrm{w}), 2934(\mathrm{w}), 1717(\mathrm{~s}), 1603(\mathrm{~m}), 1514(\mathrm{w}), 1450(\mathrm{~m}), 1391(\mathrm{~m})$, 1317 (m), 1246 (s), $1142(\mathrm{~m}), 1123(\mathrm{w}), 1040(\mathrm{w}), 1001(\mathrm{w}), 918(\mathrm{w}), 853(\mathrm{w}), 829(\mathrm{~m}), 764(\mathrm{~m}), 721(\mathrm{w}), 673$ $(\mathrm{m}), 542(\mathrm{w})$.

\section{Synthesis of the MOFs}

\subsection{UHM-25-Ala-Boc (11)}

For the synthesis of bulk material, tetracarboxylic acid $5 \mathbf{a}(377 \mathrm{mg}, 0.58 \mathrm{mmol}, 1$ eq.) was dissolved in DMF $(24 \mathrm{~mL})$. To this solution nitric acid $(20 \%, 1.9 \mathrm{~mL})$, and then an aqueous solution of copper nitrate trihydrate $\left(\mathrm{c}=100 \mathrm{~g} / \mathrm{L}, 8.8 \mathrm{~mL}, 88 \mathrm{mg} \mathrm{Cu}\left(\mathrm{NO}_{3}\right)_{2} \cdot 3 \mathrm{H}_{2} \mathrm{O}, 0.4 \mathrm{mmol}, 0.6 \mathrm{eq}\right.$.) was added. The reaction mixture was placed in an oven at $50^{\circ} \mathrm{C}$ for three days. The mother liquor was decanted and the crystals suspended in fresh DMF. This procedure was repeated twice after 24 hours each. DMF was then replaced with THF in the same manner. The solvent-exchanged MOF was kept under THF and stored in a closed container.

A fraction of the MOF was digested in diluted hydrochloric acid to reisolate the linker for spectroscopic measurements: HRMS (ESI+): $\frac{m}{z}=678.1918$ (calc. for $\mathrm{MNa}^{+}: 678.1951$ ); opt. rot.: $[\alpha]_{\mathrm{D}}^{25}=-18.7$ $(\mathrm{c}=0.33 \mathrm{~g} / 100 \mathrm{~mL}, \mathrm{MeOH})$.

${ }^{1} \mathrm{H}-\mathrm{NMR}\left(300 \mathrm{MHz}, \mathrm{CD}_{3} \mathrm{OD}\right): \delta[\mathrm{ppm}]=8.60-8.58(\mathrm{~m}, 2 \mathrm{H}, \mathrm{H} 16), 8.42\left(\mathrm{~d}, 2 \mathrm{H}^{4} J_{\mathrm{H}-\mathrm{H}}=1.7 \mathrm{~Hz}, \mathrm{H} 14 \mathrm{~A}\right)$, $8.49\left(\mathrm{~d}, 2 \mathrm{H},{ }^{4} J_{\mathrm{H}-\mathrm{H}}=1.6 \mathrm{~Hz}, \mathrm{H} 14 \mathrm{~B}\right), 7.76-7.56(\mathrm{~m}, 8 \mathrm{H}, \mathrm{H} 10, \mathrm{H} 11), 4.85\left(\mathrm{q}, 1 \mathrm{H}^{3} \mathrm{~J}_{\mathrm{H}-\mathrm{H}}=6.5 \mathrm{~Hz}, \mathrm{H} 2\right), 1.32(\mathrm{~s}$, $9 \mathrm{H}, \mathrm{H} 8), 1.13\left(\mathrm{~d}, 3 \mathrm{H},{ }^{3} J_{\mathrm{H}-\mathrm{H}}=6.5 \mathrm{~Hz}, \mathrm{H} 3\right)$.

IR (ATR): $\widetilde{v}\left[\mathrm{~cm}^{-1}\right]=2979(\mathrm{w}), 2929(\mathrm{w}), 2873(\mathrm{w}), 1768(\mathrm{~m}), 1704(\mathrm{w}), 1650(\mathrm{~s}), 1631(\mathrm{~s}), 1591(\mathrm{~m}), 1512$ (m), $1442(\mathrm{~m}), 1407(\mathrm{~m}), 1369(\mathrm{~s}), 1297(\mathrm{~m}), 1247(\mathrm{~m}), 1159(\mathrm{~m}), 1101(\mathrm{~m}), 1058(\mathrm{~m}), 1031(\mathrm{~m}), 989(\mathrm{~m}), 916$ (m), $931(\mathrm{~m}), 771(\mathrm{~m}), 729(\mathrm{~m}), 663(\mathrm{~m})$.

\subsubsection{Nitrogen Physisorption Measurement}

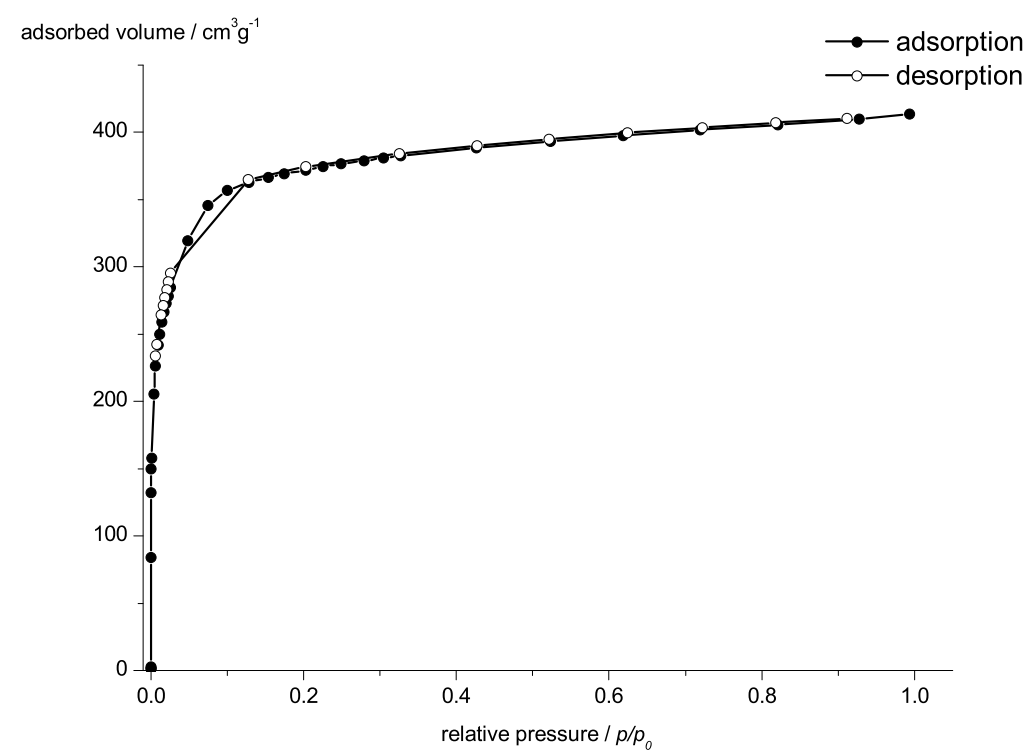

Figure S8 $-\mathrm{N}_{2}$ physisorption of UHM-25-Ala-Boc (11) after activation with supercritical $\mathrm{CO}_{2}$.

$S_{\text {BET }}=1474 \mathrm{~m}^{2} \mathrm{~g}^{-1}$ (determined for $p / p_{0}=0.016-0.100$ )

$V_{\text {micropore }}=0.58 \mathrm{~cm}^{3} \mathrm{~g}^{-1}\left(\right.$ determined at $\left.p / p_{0}=0.20\right)$ 


\subsubsection{Thermogravimetric Analysis}

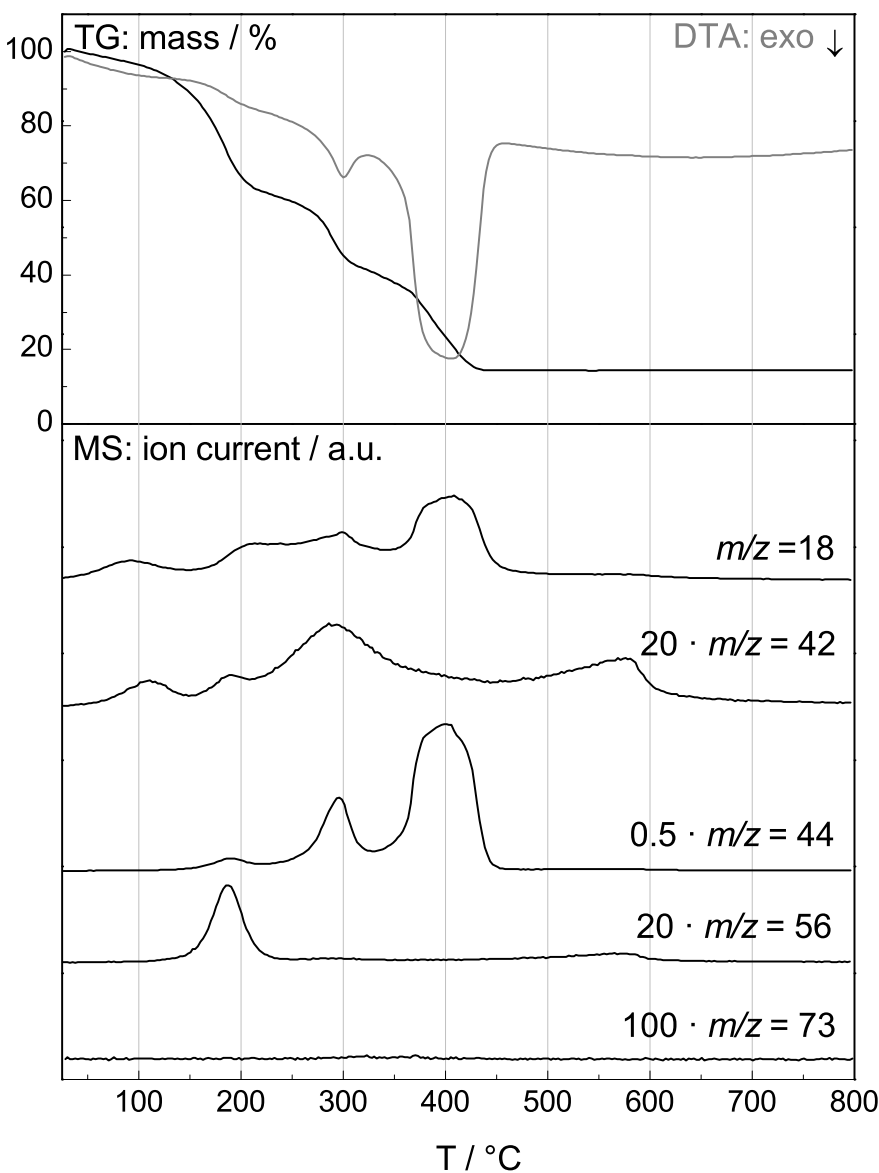

\begin{tabular}{rrr}
\hline $\begin{array}{c}\Delta \mathrm{T} \\
{ }^{\circ} \mathrm{C}\end{array}$ & $\begin{array}{r}\Delta \mathrm{m} \\
\%\end{array}$ & $\begin{array}{r}\text { fragments } \\
\mathrm{m} / \mathrm{z}\end{array}$ \\
\hline $25-145$ & 11 & 18,42 \\
$145-240$ & 28 & 44,56 \\
$240-330$ & 21 & $18,42,44$ \\
$338-432$ & 25 & 18,44 \\
\hline
\end{tabular}

Figure S9 - TG/MS analysis of UHM-25-Ala-Boc (11), weight loss (black) and DTA (grey) are plotted in the upper part and in the lower part of the diagram the characteristic single ion currents are shown for $\frac{m}{z}=18\left(\mathrm{H}_{2} \mathrm{O}\right), 42$ (THF), $44\left(\mathrm{CO}_{2}\right), 56$ (isobutene), and 73 (DMF).

\subsubsection{Crystal Structure Determination and Refinement}

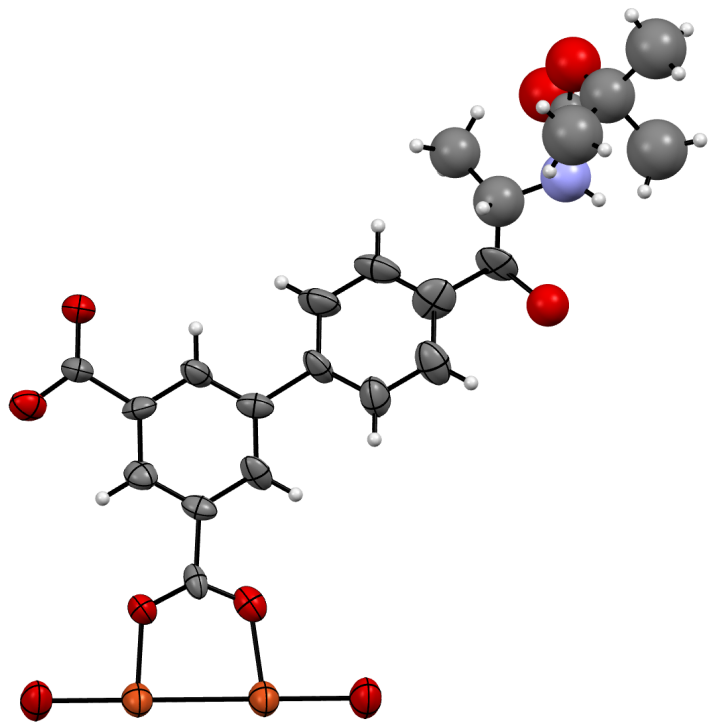

Figure S10 - ORTEP-like plot of the asymmetric unit of UHM-25-Ala-Boc (orange: copper, red: oxygen, light blue: nitrogen, grey: carbon, white: hydrogen; probability level $15 \%$ ). 
X-ray single crystal diffraction for UHM-25-Ala-Boc (11) was collected on an Oxford Diffraction (Agilent Technologies) SuperNova diffractometer at $100(2) \mathrm{K}$ with $\mathrm{Cu} K \alpha$ radiation $(154.178 \mathrm{pm})$ by $\omega$ scanning mode. The program CrysAlisPro was used for integration of the diffraction profiles and absorption corrections. The structure was solved by direct methods using the SHELXS program of the SHELXTL package and refined by full-matrix least-squares methods with SHELXL against $|F|^{2}$. All non-hydrogen atoms -except for the chiral moiety on the linker molecule- were refined with anisotropic displacement parameters. A high degree of disorder has been found for the chiral moiety on the organic secondary building unit (SBU), which can be ascribed to statistical disorder of the linker within the framework and the conformational flexibility of the substituent. Therefore, the chiral moiety in UHM-25-Ala-Boc has been modeled as a substituent with chemically reasonable bond lengths and angles but without any conformational disorder. The atomic positions of this group were held fixed during the refinement. Only one possible site of the severely disordered substituent was taken into account with small s.o.f. values. Note, without the chiral substitutent the framework posseses almost the symmetry of the space group $P m \overline{3} m$.

SQUEEZE/PLATON ${ }^{5}$ was used to account for the electron density from disordered copper atoms and $\mathrm{N}, \mathrm{N}$-dimethylformamide (DMF) solvent molecules, which could not be resolved. The contributions of 2766.3 electrons were removed from the unit cell contents. In the space group P432 2766.3/24=115.3 electrons were removed from the asymmetric unit. With $Z$ being 12, 1383.6 electrons would be associated with one formula unit, consistent with 0.5 copper atoms and 57 DMF solvent molecules per unit cell.

\begin{tabular}{ll}
\hline identification code & UHM-25-Ala-Boc \\
\hline empirical formula & C36 H32 Cu2 N1 O13 \\
formular weight & $870.70 \mathrm{~g} / \mathrm{mol}$ \\
temperature & $100(2) \mathrm{K}$ \\
wavelength & $154.178 \mathrm{pm}, \mathrm{Cu} \mathrm{K \alpha}$ \\
crystal system & cubic \\
space group & $P 432$ \\
unit cell dimensions & $a=2894.16(4) \mathrm{pm} \alpha=90^{\circ}$ \\
& $b=2894.16(4) \mathrm{pm} \beta=90^{\circ}$ \\
& $\mathrm{c}=2894.16(4) \mathrm{pm} \gamma=90^{\circ}$ \\
volume & $24242.0(5) \AA^{3}$ \\
$Z$ & 12 \\
calculated density & $0.928 \mathrm{~g} / \mathrm{cm}^{3}$ \\
absorption coefficient & $\mu=0.91 \mathrm{~mm}^{-1}$ \\
$F(000)$ & 5004 \\
crystal size & $0.12 \times 0.12 \times 0.08 \mathrm{~mm}{ }^{3}$ \\
$\theta$ range for data collection & 3.4 to 37.0 \\
index ranges & $h=-22 \rightarrow 22, k=-22 \rightarrow 22, l=-22 \rightarrow 22$ \\
reflections collected & 73768 \\
independent reflections & 2046 \\
observed reflections $(I>2 \sigma(I))$ & $1668\left(R_{\text {int }}=0.068\right)$ \\
completeness to $\theta=37.0$ & $99.67 \%$ \\
absorption correction & multi-scan \\
refinement method & Full-matrix least squares on $F^{2}$ \\
data / restraints $/$ parameters & $2046 / 67 / 198$ \\
goodness-of-fit on $F^{2}$ & $S=1.10$ \\
final $R$ indices $[I>\sigma(I)]$ & $R 1=0.077 w R 2=0.241$ \\
$R$ indices (all data) & $R 1=0.086, w R 2=0.242$ \\
largest diff. peak and hole & $\Delta \rho_{\text {max }} 0.27 \mathrm{e} / \AA^{3}, \Delta \rho_{\text {min }}-0.24 \mathrm{e} / \AA^{3}$ \\
\hline
\end{tabular}

To evaluate the phase purity of the bulk phase of UHM-25-Ala-Boc, PXRD data was collected and compared with a simulated PXRD pattern. The simulated pattern was calculated for a hypothetical structure in which the chiral moiety was excluded so that the crystal structure contains only the backbone of the linker. After this simplification of the structure, the patterns of the bulk phase and the simulated pattern are in reasonable agreement (see Figure S11). However, significant deviations and in particular additional reflections become visible in the simulated XRD pattern based on a structural model that explicitly includes the chiral moiety, thereby indirectly proving the severe disorder of the chiral group in the real sample. 


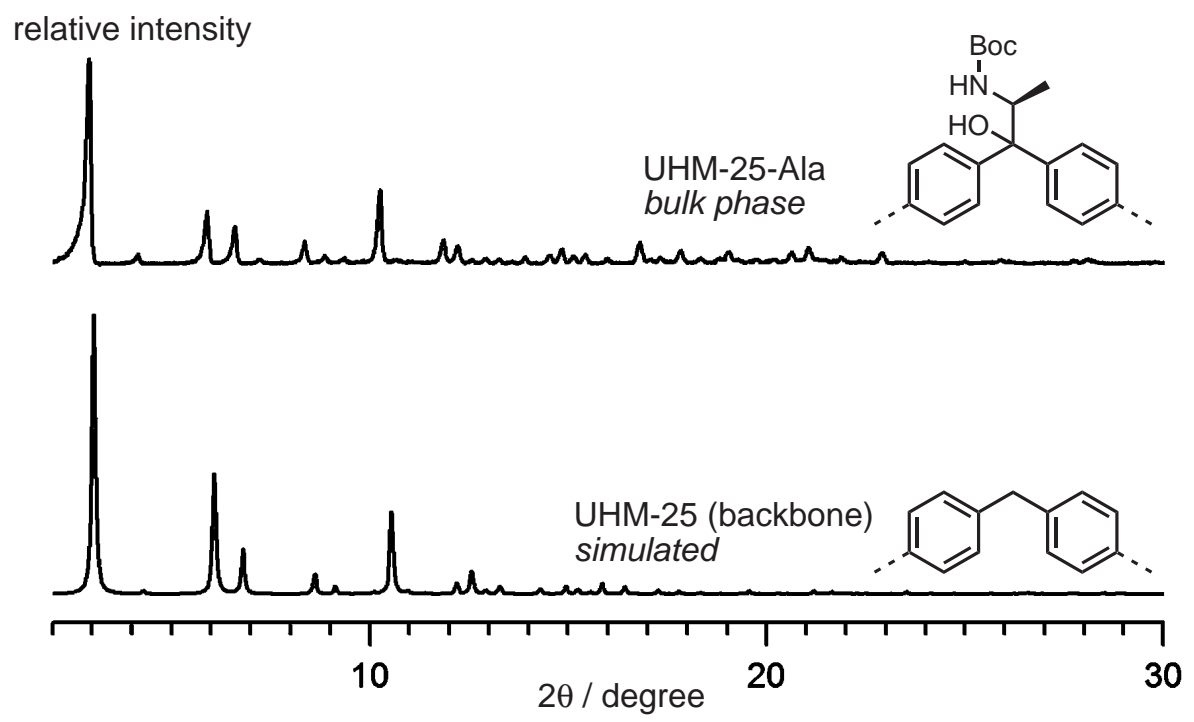

Figure S11 - Experimental PXRD for UHM-25-Ala-Boc as-synthesized (top) and a calculated PXRD pattern for the UHM-25 backbone bearing no substituents on the $\mathrm{sp}^{3}$-hybridized carbon of the organic SBU (bottom).

\subsubsection{Investigations on the Structural Integrity of the Linker}

To investigate and prove the stability of the chiral linker after the MOF synthesis, the reaction product UHM-25-Ala-Boc was digested in diluted $\mathrm{HCl}$. This allowed the reisolation of the linker which could then be examined by spectroscopic methods. The integrity of the linker was independently verified by ${ }^{1} \mathrm{H}-\mathrm{NMR}$ spectroscopy and mass spectrometry. The retention of chirality in the linker was confirmed by polarimetry measurements of the reisolated material, which showed only a slight decrease of optical activity.

\subsection{UHM-25-Val-Boc (12)}

The reaction was performed according to the procedure described in Section 4.1

IR (ATR): $\widetilde{v}\left[\mathrm{~cm}^{-1}\right]=2964(\mathrm{w}), 2875(\mathrm{w}), 1729(\mathrm{~m}), 1612(\mathrm{~m}), 1554(\mathrm{~m}), 1442(\mathrm{~m}), 1400(\mathrm{~m}), 1365$ (s), $1294(\mathrm{~m}), 1236$ (m), 1108 (m), $1043(\mathrm{~m}), 997$ (m), 916 (m), 885 (m), $829(\mathrm{~m}), 767$ (m), $730(\mathrm{~m}), 686(\mathrm{~m})$.

\subsubsection{Nitrogen Physisorption Measurement}

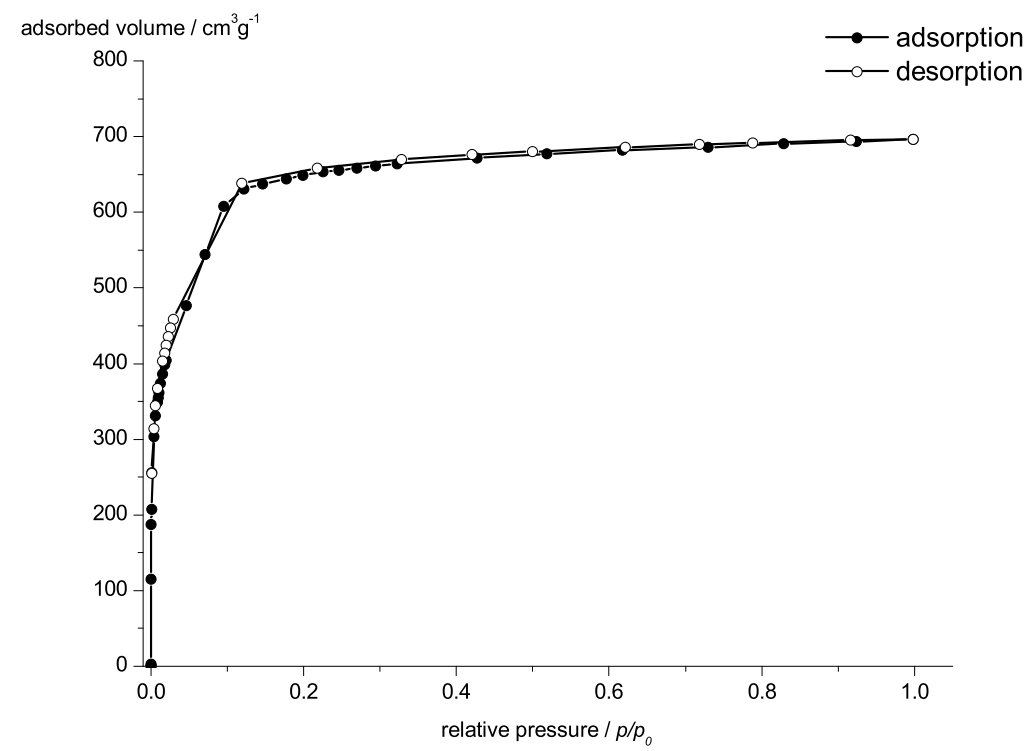

Figure S12 $-\mathrm{N}_{2}$ physisorption of UHM-25-Val-Boc (12) after activation with supercritical $\mathrm{CO}_{2}$. 
$S_{\mathrm{BET}}=1922 \mathrm{~m}^{2} \mathrm{~g}^{-1}$ (determined for $p / p_{0}=0.009-0.020$ )

$V_{\text {micropore }}=1.00 \mathrm{~cm}^{3} \mathrm{~g}^{-1}\left(\right.$ determined at $\left.p / p_{0}=0.20\right)$

\subsubsection{Thermogravimetric Analysis}

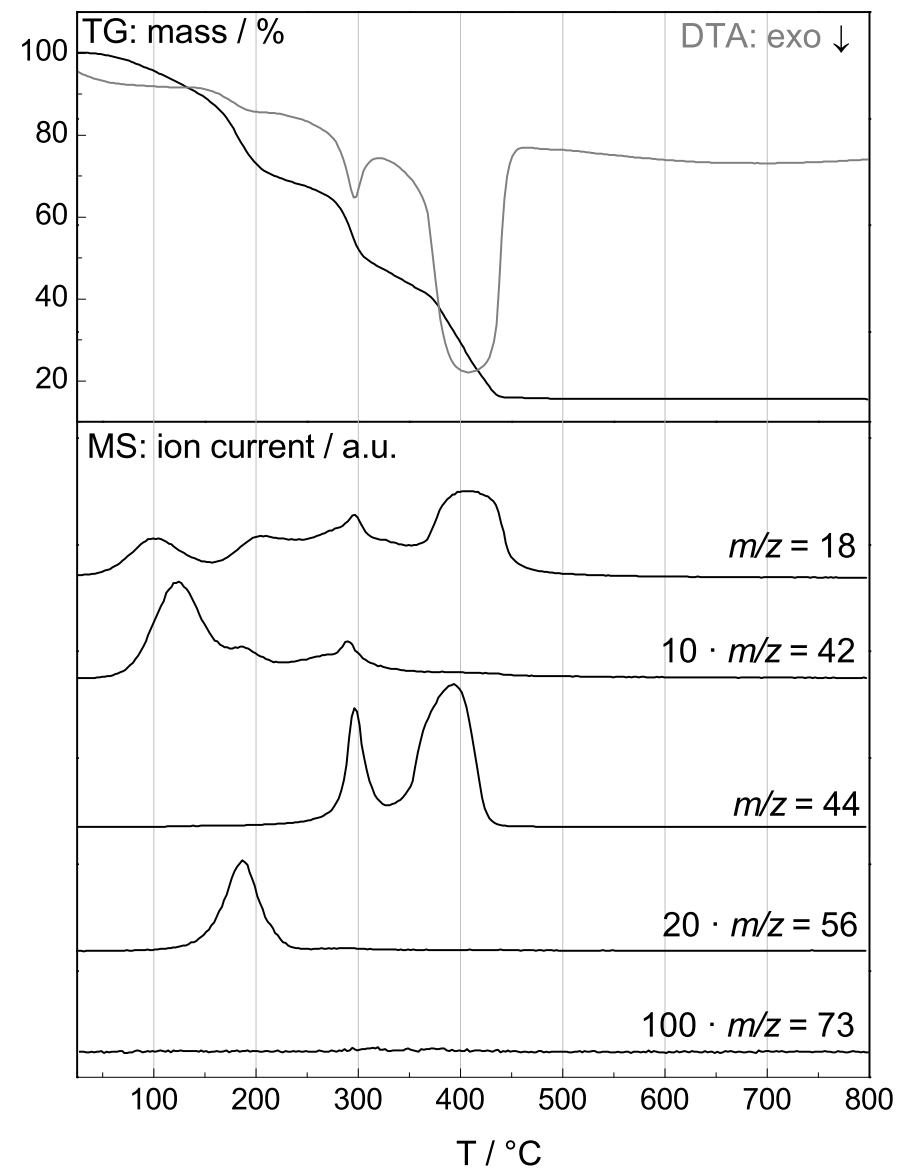

\begin{tabular}{rrr}
\hline $\begin{array}{r}\Delta \mathrm{T} \\
{ }^{\circ} \mathrm{C}\end{array}$ & $\begin{array}{r}\Delta \mathrm{m} \\
\%\end{array}$ & $\begin{array}{r}\text { fragments } \\
\mathrm{m} / \mathrm{z}\end{array}$ \\
\hline $25-150$ & 11 & 18,42 \\
$150-240$ & 19 & 44,56 \\
$240-338$ & 23 & $18,42,44$ \\
$338-443$ & 29 & 18,44 \\
\hline
\end{tabular}

Figure S13 - TG/MS analysis of UHM-25-Val-Boc (12), weight loss (black) and DTA (grey) are plotted in the upper part and in the lower part of the diagram the characteristic single ion currents are shown for $\frac{m}{z}=18\left(\mathrm{H}_{2} \mathrm{O}\right), 42(\mathrm{THF}), 44\left(\mathrm{CO}_{2}\right), 56$ (isobutene), and $73(\mathrm{DMF})$.

\subsection{UHM-25-Leu-Boc (13)}

The reaction was performed according to the procedure described in Section 4.1

IR (ATR): $\widetilde{v}\left[\mathrm{~cm}^{-1}\right]=2959(\mathrm{w}), 2931(\mathrm{w}), 2869(\mathrm{w}), 1708(\mathrm{w}), 1635(\mathrm{~m}), 1558(\mathrm{~m}), 1508(\mathrm{w}), 1444(\mathrm{~m})$, 1402 (m), 1365 (s), 1296 (m), 1247 (m), 1162 (m), 1049 (m), 912 (m), 887 (m), 831 (m), 771 (m), 729 (s), 684 (m). 


\subsubsection{Nitrogen Physisorption Measurement}

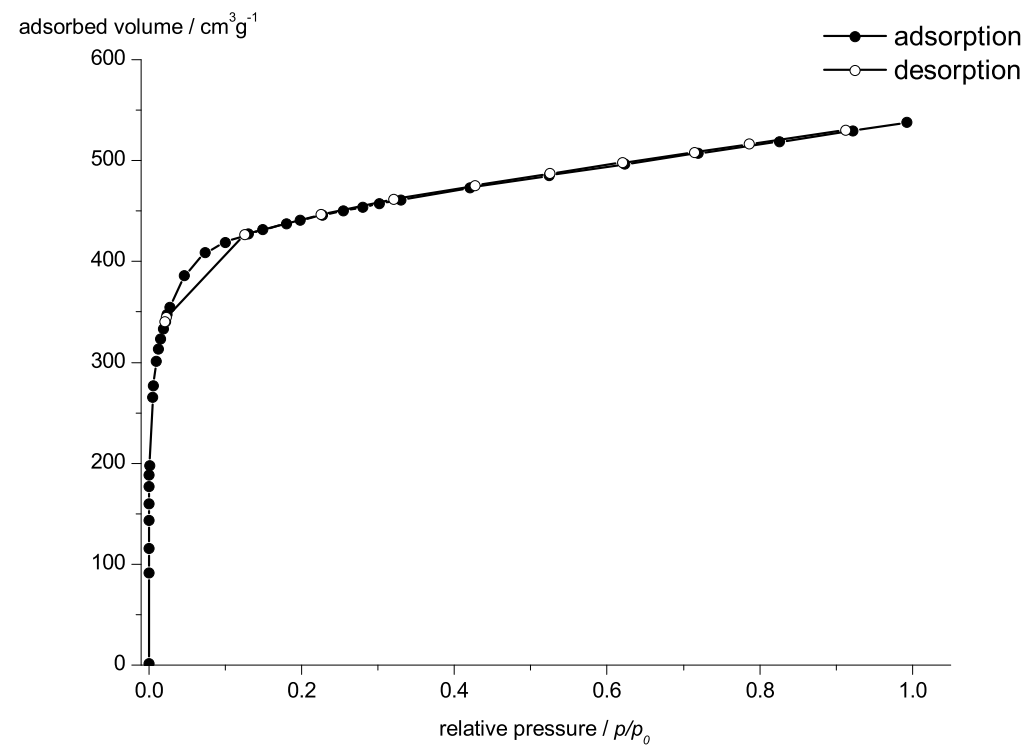

Figure S14 $-\mathrm{N}_{2}$ physisorption of UHM-25-Leu-Boc (13) after activation with supercritical $\mathrm{CO}_{2}$.

$S_{\mathrm{BET}}=1474 \mathrm{~m}^{2} \mathrm{~g}^{-1}$ (determined for $p / p_{0}=0.016-0.074$ )

$V_{\text {micropore }}=0.68 \mathrm{~cm}^{3} \mathrm{~g}^{-1}\left(\right.$ determined at $\left.p / p_{0}=0.20\right)$ 


\subsubsection{Thermogravimetric Analysis}

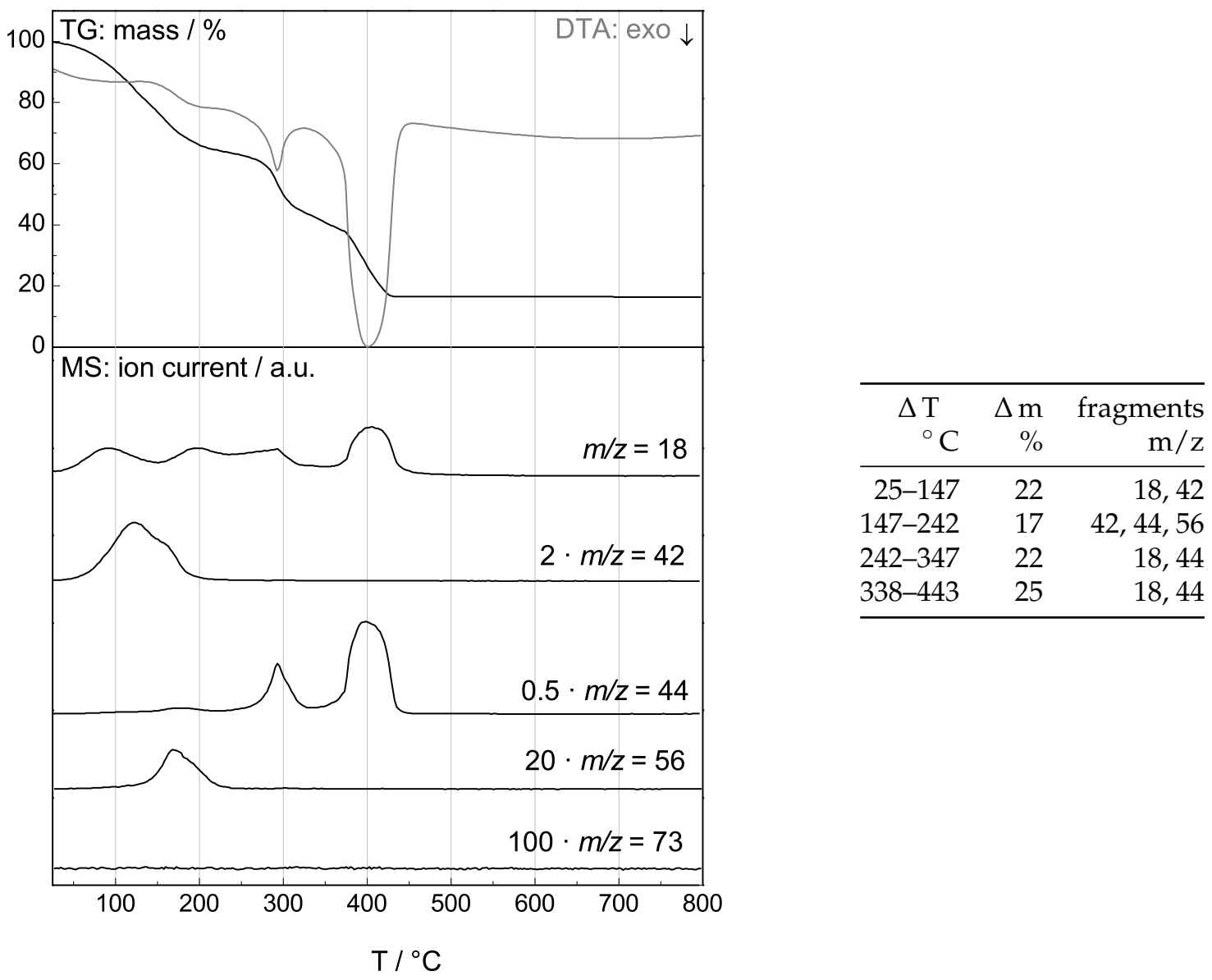

Figure S15 - TG/MS analysis of UHM-25-Leu-Boc (13), weight loss (black) and DTA (grey) are plotted in the upper part and in the lower part of the diagram the characteristic single ion currents are shown for $\frac{m}{z}=18\left(\mathrm{H}_{2} \mathrm{O}\right), 42(\mathrm{THF}), 44\left(\mathrm{CO}_{2}\right), 56$ (isobutene), and $73(\mathrm{DMF})$.

\subsection{UHM-25-Ala (14)}

The reaction was performed according to the procedure described in Section 4.1

IR (ATR): $\widetilde{v}\left[\mathrm{~cm}^{-1}\right]=2927(\mathrm{w}), 2987,1710(\mathrm{w}), 1602(\mathrm{~m}), 1556(\mathrm{~s}), 1444(\mathrm{~m}), 1400(\mathrm{~m}), 1359(\mathrm{~s}), 1286$ $(\mathrm{m}), 1110(\mathrm{w}), 1037(\mathrm{w}), 916(\mathrm{~m}), 833(\mathrm{~m}), 765(\mathrm{~s}), 721(\mathrm{~s})$. 


\subsubsection{Nitrogen Physisorption Measurement}

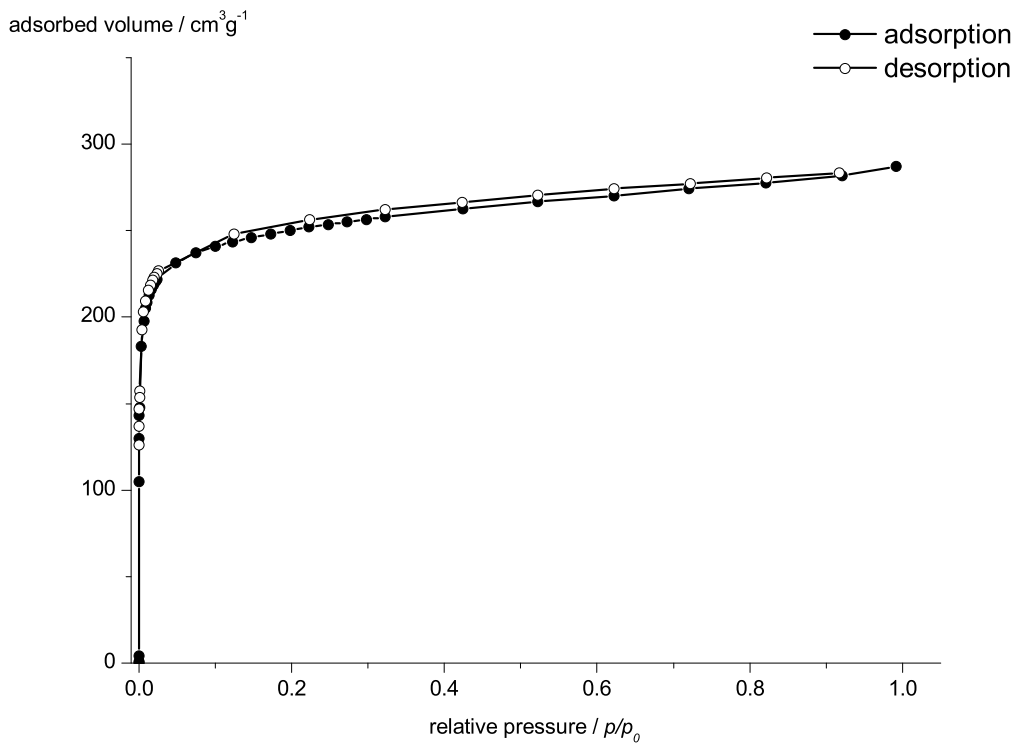

Figure S16 - $\mathrm{N}_{2}$ physisorption of UHM-25-Ala (14) after activation with supercritical $\mathrm{CO}_{2}$.

$S_{\mathrm{BET}}=975.7 \mathrm{~m}^{2} \mathrm{~g}^{-1}$ (determined for $\left.p / p_{0}=0.010-0.048\right)$

$V_{\text {micropore }}=0.39 \mathrm{~cm}^{3} \mathrm{~g}^{-1}\left(\right.$ determined at $\left.p / p_{0}=0.20\right)$ 


\subsubsection{Thermogravimetric Analysis}

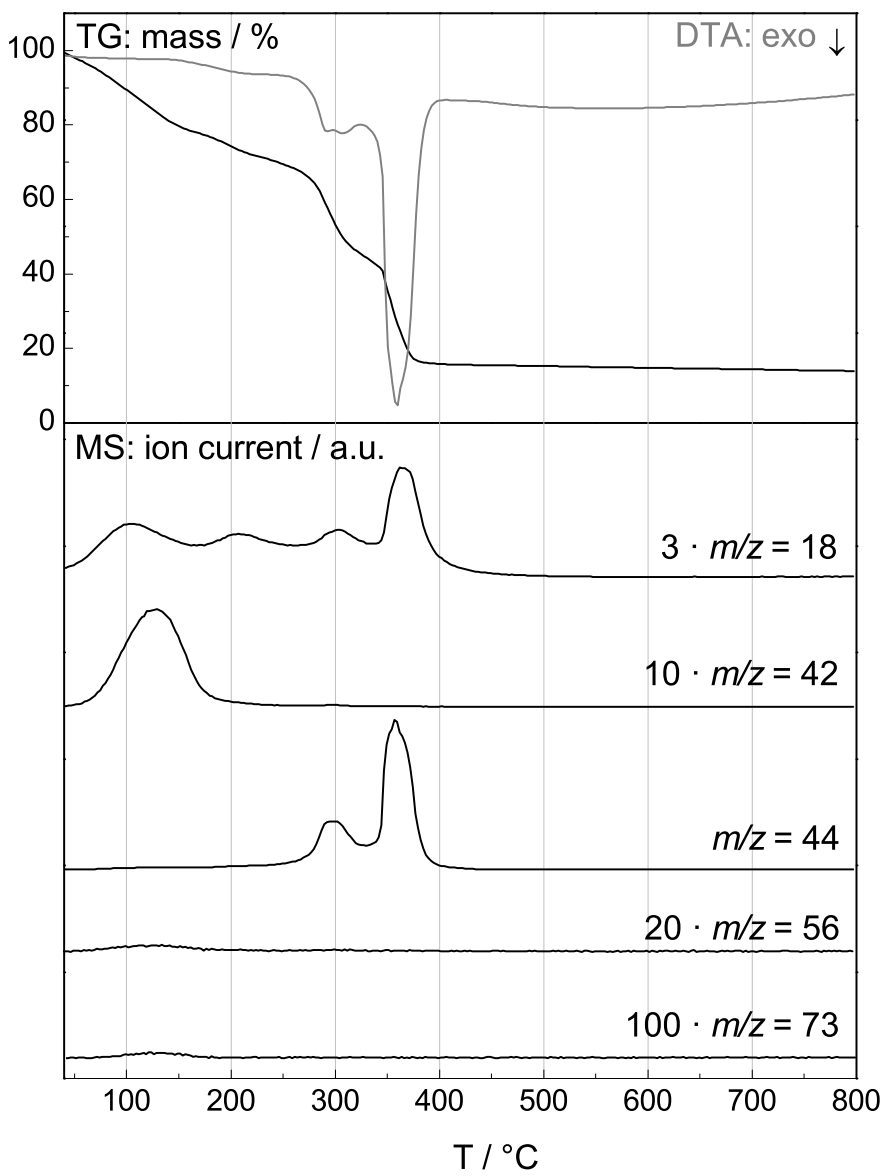

\begin{tabular}{rrr}
\hline $\begin{array}{c}\Delta \mathrm{T} \\
{ }^{\circ} \mathrm{C}\end{array}$ & $\begin{array}{r}\Delta \mathrm{m} \\
\%\end{array}$ & $\begin{array}{r}\text { fragments } \\
\mathrm{m} / \mathrm{z}\end{array}$ \\
\hline $25-217$ & 28 & 42,44 \\
$217-327$ & 27 & 18,44 \\
$327-380$ & 30 & 18,44 \\
\hline
\end{tabular}

Figure S17 - TG/MS analysis of UHM-25-Ala (14), weight loss (black) and DTA (grey) are plotted in the upper part and in the lower part of the diagram the characteristic single ion currents are shown for $\frac{m}{z}=18\left(\mathrm{H}_{2} \mathrm{O}\right), 42$ (THF), $44\left(\mathrm{CO}_{2}\right), 56$ (isobutene), and 73 (DMF).

\subsection{UHM-25-Val (15)}

The reaction was performed according to the procedure described in Section 4.1

IR (ATR): $\widetilde{v}\left[\mathrm{~cm}^{-1}\right]=2959(\mathrm{w}), 2931(\mathrm{w}), 2869(\mathrm{w}), 1708(\mathrm{w}), 1635(\mathrm{~m}), 1558(\mathrm{~m}), 1508(\mathrm{w}), 1444(\mathrm{~m})$, $1402(\mathrm{~m}), 1365$ (s), $1296(\mathrm{~m}), 1247$ (m), $1162(\mathrm{~m}), 1049(\mathrm{~m}), 912(\mathrm{~m}), 887(\mathrm{~m}), 831(\mathrm{~m}), 771(\mathrm{~m}), 729(\mathrm{~s}), 684$ (m). 


\subsubsection{Nitrogen Physisorption Measurement}

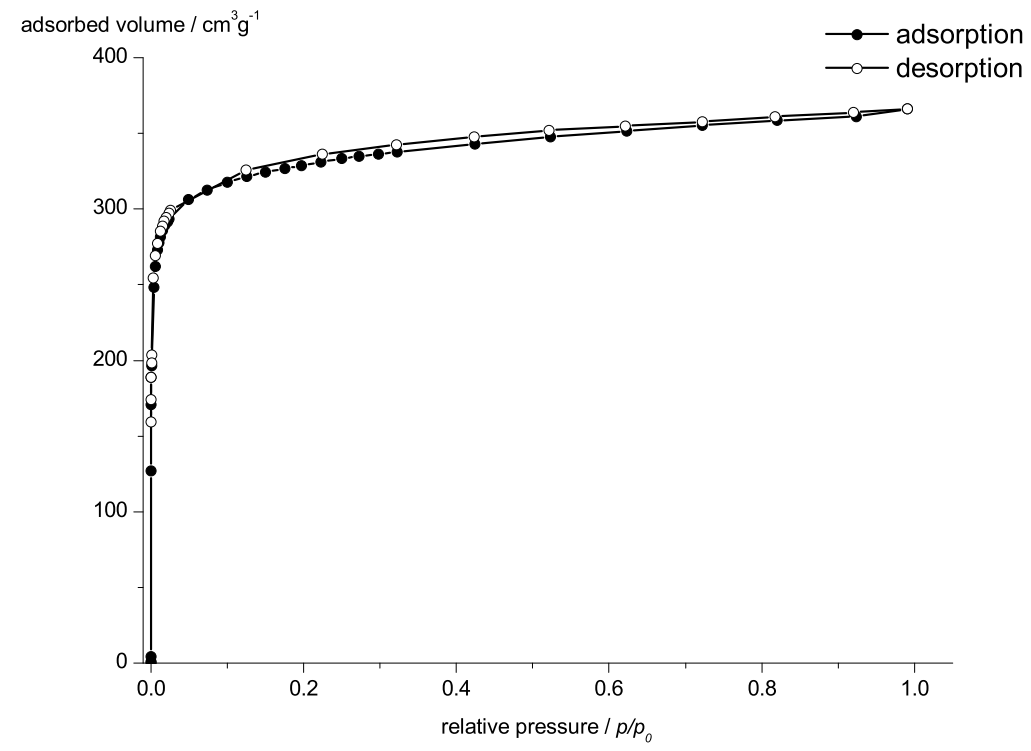

Figure S18 $-\mathrm{N}_{2}$ physisorption of UHM-25-Val (15) after activation with supercritical $\mathrm{CO}_{2}$.

$S_{\mathrm{BET}}=1286 \mathrm{~m}^{2} \mathrm{~g}^{-1}$ (determined for $p / p_{0}=0.011-0.049$ )

$V_{\text {micropore }}=0.51 \mathrm{~cm}^{3} \mathrm{~g}^{-1}\left(\right.$ determined at $\left.p / p_{0}=0.20\right)$ 


\subsubsection{Thermogravimetric Analysis}

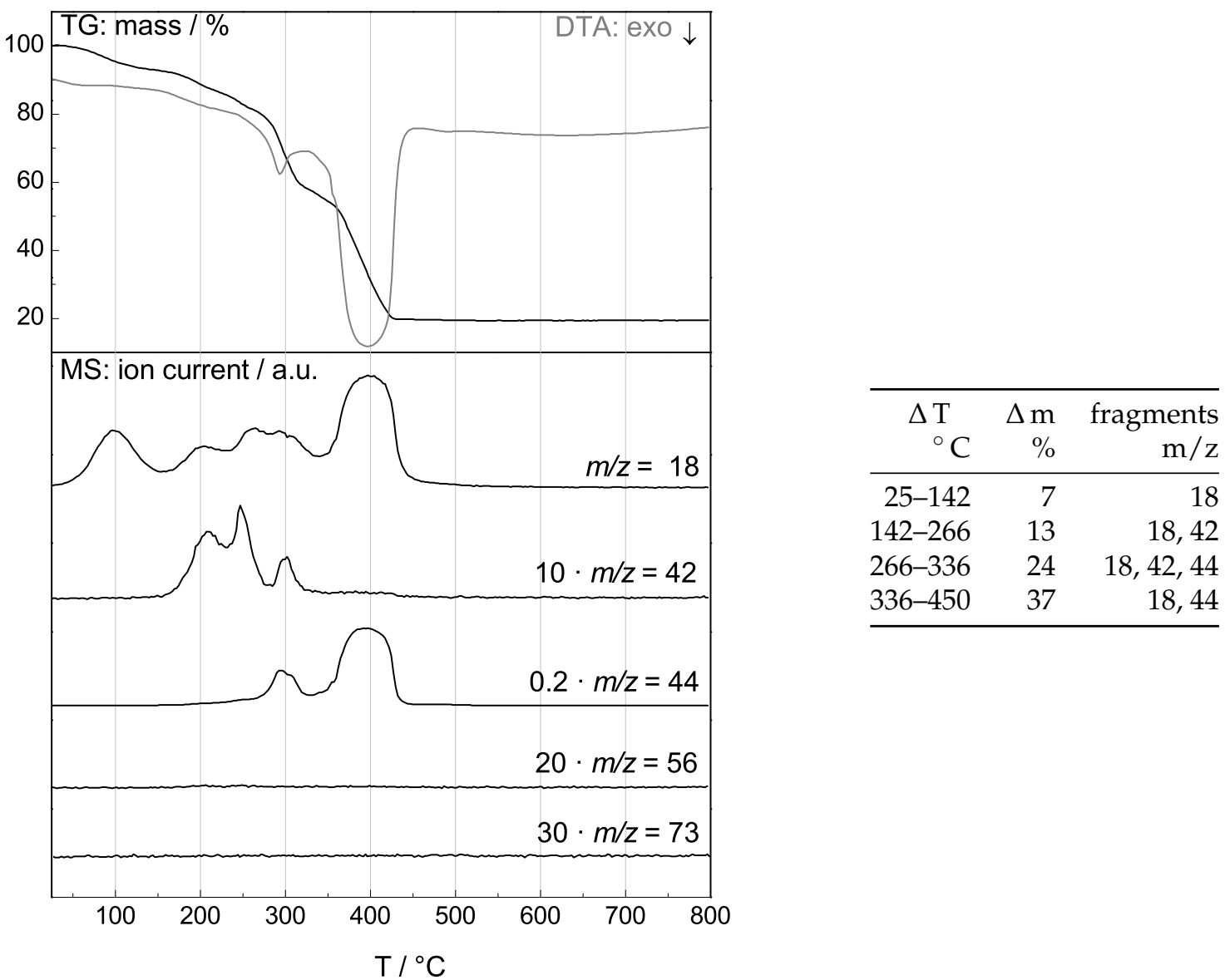

Figure S19 - TG/MS analysis of UHM-25-Val (15), weight loss (black) and DTA (grey) are plotted in the upper part and in the lower part of the diagram the characteristic single ion currents are shown for $\frac{m}{z}=18\left(\mathrm{H}_{2} \mathrm{O}\right), 42$ (THF), $44\left(\mathrm{CO}_{2}\right), 56$ (isobutene), and 73 (DMF).

\subsection{UHM-25-Leu (16)}

The reaction was performed according to the procedure described in Section 4.1

IR (ATR): $\widetilde{v}\left[\mathrm{~cm}^{-1}\right]=2956(\mathrm{w}), 2927(\mathrm{w}), 2869(\mathrm{w}), 1714(\mathrm{w}), 1608(\mathrm{w}), 1554(\mathrm{~m}), 1512(\mathrm{w}), 1442(\mathrm{~m})$, $1404(\mathrm{~m}), 1359(\mathrm{~s}), 1294(\mathrm{~m}), 1245(\mathrm{~m}), 1135(\mathrm{w}), 1031(\mathrm{w}), 912(\mathrm{w}), 831(\mathrm{~m}), 769(\mathrm{~m}), 723(\mathrm{~m}), 684(\mathrm{~m})$. 


\subsubsection{Nitrogen Physisorption Measurement}

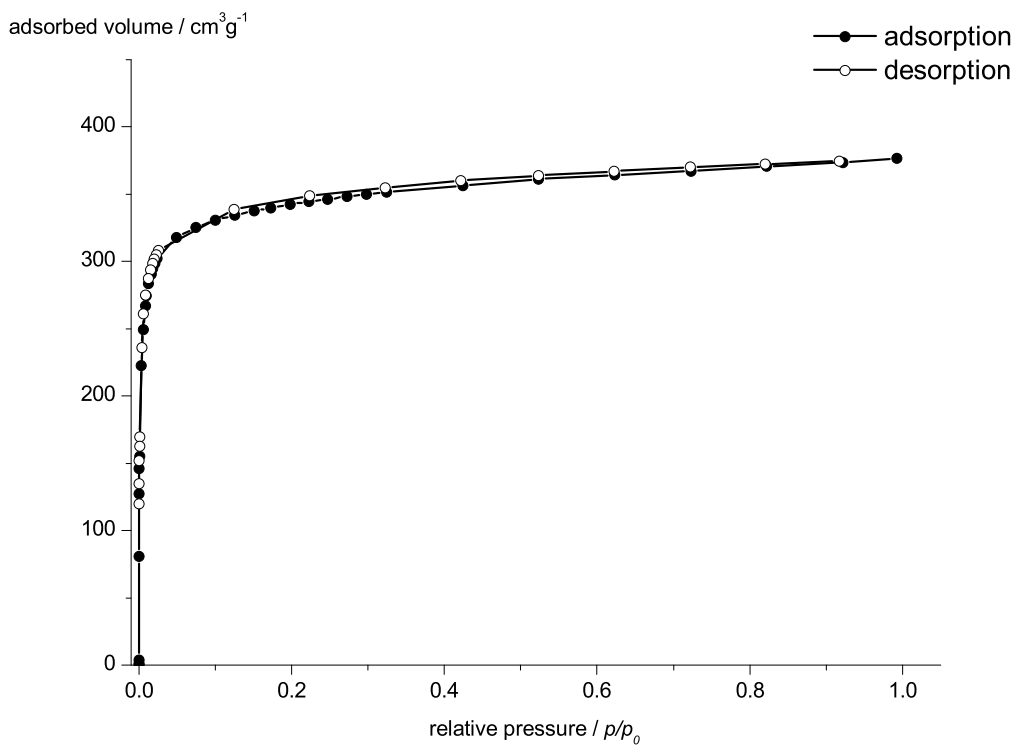

Figure S20 - $\mathrm{N}_{2}$ physisorption of UHM-25-Leu (16) after activation with supercritical $\mathrm{CO}_{2}$.

$S_{\mathrm{BET}}=1351 \mathrm{~m}^{2} \mathrm{~g}^{-1}$ (determined for $p / p_{0}=0.010-0.049$ )

$V_{\text {micropore }}=0.53 \mathrm{~cm}^{3} \mathrm{~g}^{-1}$ (determined at $\left.p / p_{0}=0.20\right)$ 


\subsubsection{Thermogravimetric Analysis}

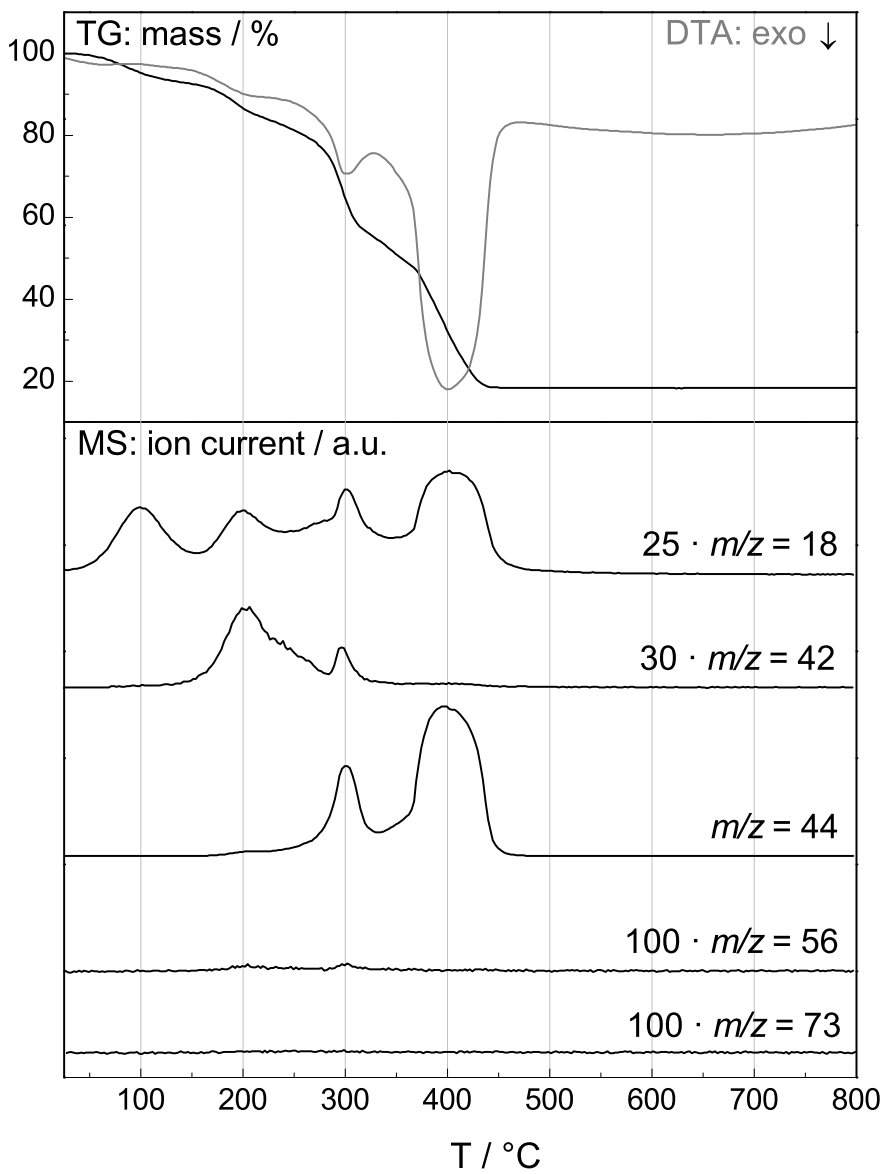

\begin{tabular}{rrr}
\hline $\begin{array}{c}\Delta \mathrm{T} \\
{ }^{\circ} \mathrm{C}\end{array}$ & $\begin{array}{r}\Delta \mathrm{m} \\
\%\end{array}$ & $\begin{array}{r}\text { fragments } \\
\mathrm{m} / \mathrm{z}\end{array}$ \\
\hline $25-123$ & 6 & 18,42 \\
$123-229$ & 10 & 18,42 \\
$229-336$ & 30 & $18,42,44$ \\
$336-436$ & 35 & 18,44 \\
\hline
\end{tabular}

Figure S21 - TG/MS analysis of UHM-25-Leu (16), weight loss (black) and DTA (grey) are plotted in the upper part and in the lower part of the diagram the characteristic single ion currents are shown for $\frac{m}{z}=18\left(\mathrm{H}_{2} \mathrm{O}\right), 42$ (THF), $44\left(\mathrm{CO}_{2}\right), 56$ (isobutene), and 73 (DMF).

\subsection{UHM-25-Pro (17)}

The reaction was performed according to the procedure described in Section 4.1

IR (ATR): $\widetilde{v}\left[\mathrm{~cm}^{-1}\right]=2975(\mathrm{w}), 2935(\mathrm{w}), 2875(\mathrm{w}), 1710(\mathrm{w}), 1612(\mathrm{~m}), 1556(\mathrm{~m}), 1510(\mathrm{w}), 1442(\mathrm{~m})$, $1402(\mathrm{~m}), 1357(\mathrm{~s}), 1296(\mathrm{~m}), 1105(\mathrm{w}), 1040(\mathrm{w}), 910(\mathrm{w}), 827(\mathrm{~m}), 767(\mathrm{~m}), 719(\mathrm{~m}), 686(\mathrm{~m}), 665(\mathrm{~m})$. 


\subsubsection{Nitrogen Physisorption Measurement}

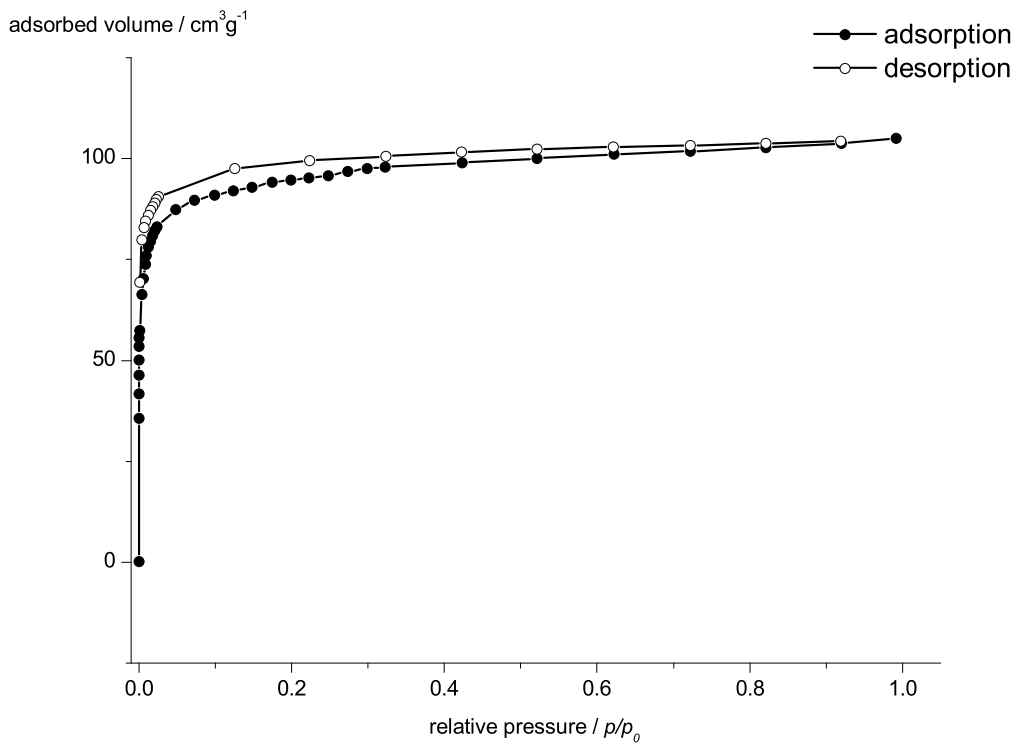

Figure S22 $-\mathrm{N}_{2}$ physisorption of UHM-25-Pro (17) after activation with supercritical $\mathrm{CO}_{2}$.

$S_{\mathrm{BET}}=371.5 \mathrm{~m}^{2} \mathrm{~g}^{-1}$ (determined for $\left.p / p_{0}=0.010-0.048\right)$

$V_{\text {micropore }}=0.15 \mathrm{~cm}^{3} \mathrm{~g}^{-1}\left(\right.$ determined at $\left.p / p_{0}=0.20\right)$ 


\subsubsection{Thermogravimetric Analysis}

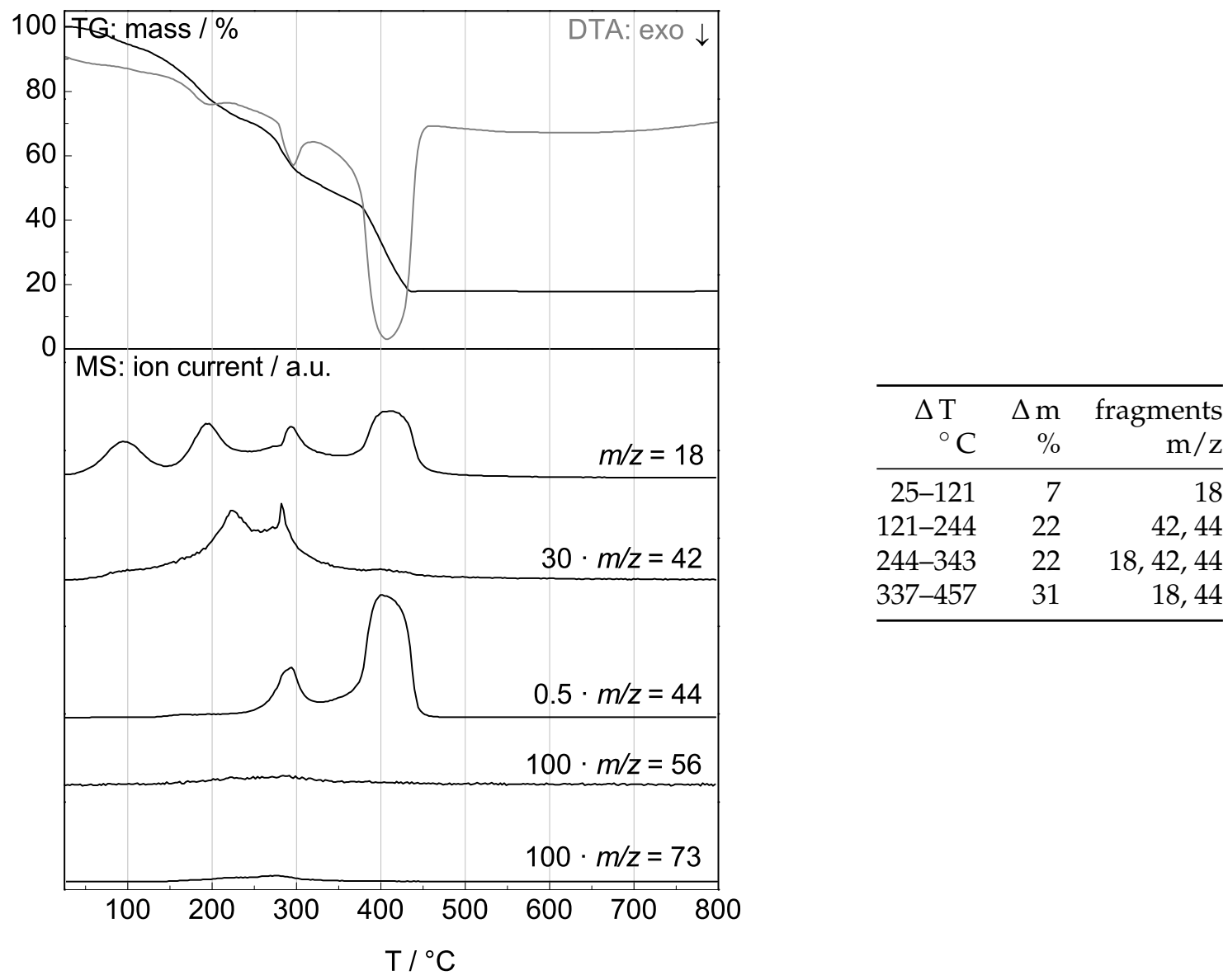

Figure S23 - TG/MS analysis of UHM-25-Pro (17), weight loss (black) and DTA (grey) are plotted in the upper part and in the lower part of the diagram the characteristic single ion currents are shown for $\frac{m}{z}=18\left(\mathrm{H}_{2} \mathrm{O}\right), 42$ (THF), $44\left(\mathrm{CO}_{2}\right), 56$ (isobutene), and 73 (DMF). 


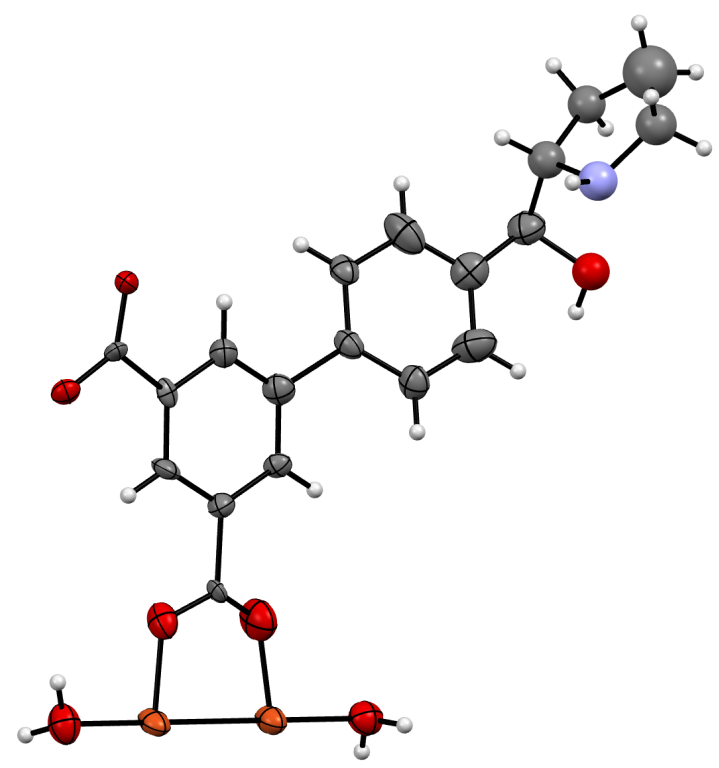

Figure S24 - ORTEP-like plot of the asymmetric unit of UHM-25-Pro (orange: copper, red: oxygen, light blue: nitrogen, grey: carbon, white: hydrogen; probability level $15 \%$ ).

X-ray single crystal diffraction for UHM-25-Pro (17) was collected on an Oxford Diffraction (Agilent Technologies) SuperNova diffractometer at $100(2) \mathrm{K}$ with $\mathrm{Cu} K \alpha$ radiation $(154.178 \mathrm{pm}$ ) by $\omega$ scanning mode. The program CrysAlisPro was used for integration of the diffraction profiles and absorption corrections. The structure was solved by direct methods using the SHELXS program of the SHELXTL package and refined by full-matrix least-squares methods with SHELXL by successive Fourier syntheses against $|F|^{2}$. All non-hydrogen atoms -except for the chiral moiety on the linker molecule- were refined with anisotropic displacement parameters. The location of the hydrogen atoms of the coordinated water molecules at the copper atoms of the paddle-wheel motif could not be assigned. A high degree of disorder has been found for the chiral moiety on the organic SBU, which can be ascribed to statistical disorder of the linker within the framework and the conformational flexibility of the substituent. Hence, this group cannot be sufficiently located from the difference Fourier map. Therefore the chiral substituent in UHM-25-Pro has been modeled as a substituent with chemically reasonable bond lengths and angles but without any conformational disorder. The atomic positions of this group were held fixed during the refinement.

SQUEEZE/PLATON ${ }^{5}$ was used to account for the electron density from disordered copper atoms and DMF solvent molecules, which could not be resolved. The contributions of 5571 electrons were removed from the unit cell contents. In the space group P432 5571/24 = 232.1 electrons were removed from the asymmetric unit. With $Z$ being $12,2785.5$ electrons would be associated with one formula unit, consistent with 0.5 copper(II) ions and 76 DMF solvent molecules per unit cell.

\begin{tabular}{ll}
\hline identification code & UHM-25-Pro \\
\hline empirical formula & $\mathrm{C} 33 \mathrm{H} 27 \mathrm{Cu} 2 \mathrm{~N} 1 \mathrm{O} 11$ \\
formular weight & $740.63 \mathrm{~g} / \mathrm{mol}$ \\
temperature & $100(2) \mathrm{K}$ \\
wavelength & $154.178 \mathrm{pm}, \mathrm{Cu} \mathrm{K \alpha}$ \\
crystal system & cubic \\
space group & $P 432$ \\
unit cell dimensions & $a=2902.61(6) \mathrm{pm} \alpha=90^{\circ}$ \\
& $b=2902.61(6) \mathrm{pm} \beta=90^{\circ}$ \\
& $c=2902.61(6) \mathrm{pm} \gamma=90^{\circ}$ \\
volume & $24454.9(15) \AA^{3}$ \\
$\mathrm{Z}$ & 12 \\
calculated density & $0.603 \mathrm{~g} / \mathrm{cm}^{3}$ \\
absorption coefficient & $\mu=0.86 \mathrm{~mm}^{-1}$ \\
$F(000)$ & 4536
\end{tabular}




\begin{tabular}{ll} 
crystal size & $0.10 \times 0.10 \times 0.09 \mathrm{~mm}^{3}$ \\
$\theta$ range for data collection & 3.4 to 45.0 \\
index ranges & $h=-14 \rightarrow 15, k=0 \rightarrow 18, l=2 \rightarrow 26$ \\
reflections collected & 160315 \\
independent reflections & 3326 \\
independent reflections $(I>2 \sigma(I))$ & $2055\left(R_{\text {int }}=0.075\right)$ \\
absorption correction & multi-scan \\
refinement method & Full-matrix least squares on $F^{2}$ \\
data / restraints / parameters & $3326 / 38 / 189$ \\
goodness-of-fit on $F^{2}$ & $S=1.09$ \\
final $R$ indices $[I>\sigma(I)]$ & $\mathrm{R} 1=0.121 \mathrm{wR} 2=0.311$ \\
$R$ indices (all data) & $R 1=0.149, w R 2=0.336$ \\
largest diff. peak and hole & $\Delta \rho_{\max } 0.76 \mathrm{e} / \AA^{3}, \Delta \rho_{\min }-0.60 \mathrm{e} / \AA^{3}$ \\
\hline &
\end{tabular}

\subsection{UHM-25-Val-Evans (18)}

The reaction was performed according to the procedure described in Section 4.1

IR (KBr): $\widetilde{v}\left[\mathrm{~cm}^{-1}\right]=3439(\mathrm{w}), 2961(\mathrm{w}), 2932(\mathrm{w}), 2878(\mathrm{w}), 1755(\mathrm{~m}), 1661(\mathrm{~s}), 1574(\mathrm{~m}), 1512(\mathrm{w})$, $1497(\mathrm{w}), 1441$ (s), $1412(\mathrm{~s}), 1387(\mathrm{~s}), 1367(\mathrm{~s}), 1298(\mathrm{w}), 1254(\mathrm{~m}), 1101(\mathrm{~m}), 1063(\mathrm{w}), 1040(\mathrm{w}), 1001(\mathrm{w})$, $918(w), 858(w), 833(w), 775(m), 729(m), 694(w), 663(w)$.

\subsubsection{Nitrogen Physisorption Measurement}

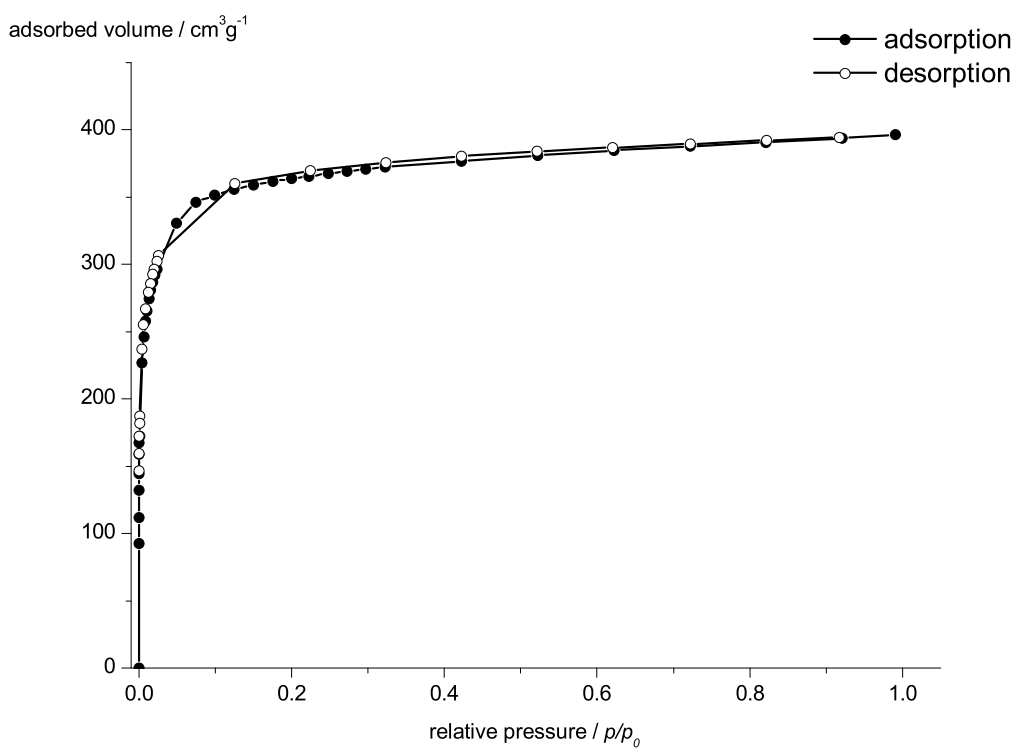

Figure S25 - $\mathrm{N}_{2}$ physisorption of UHM-25-Val-Evans (18) after activation with supercritical $\mathrm{CO}_{2}$.

$S_{\mathrm{BET}}=1454 \mathrm{~m}^{2} \mathrm{~g}^{-1}$ (determined for $p / p_{0}=0.014-0.075$ )

$V_{\text {micropore }}=0.56 \mathrm{~cm}^{3} \mathrm{~g}^{-1}$ (determined at $\left.p / p_{0}=0.20\right)$ 


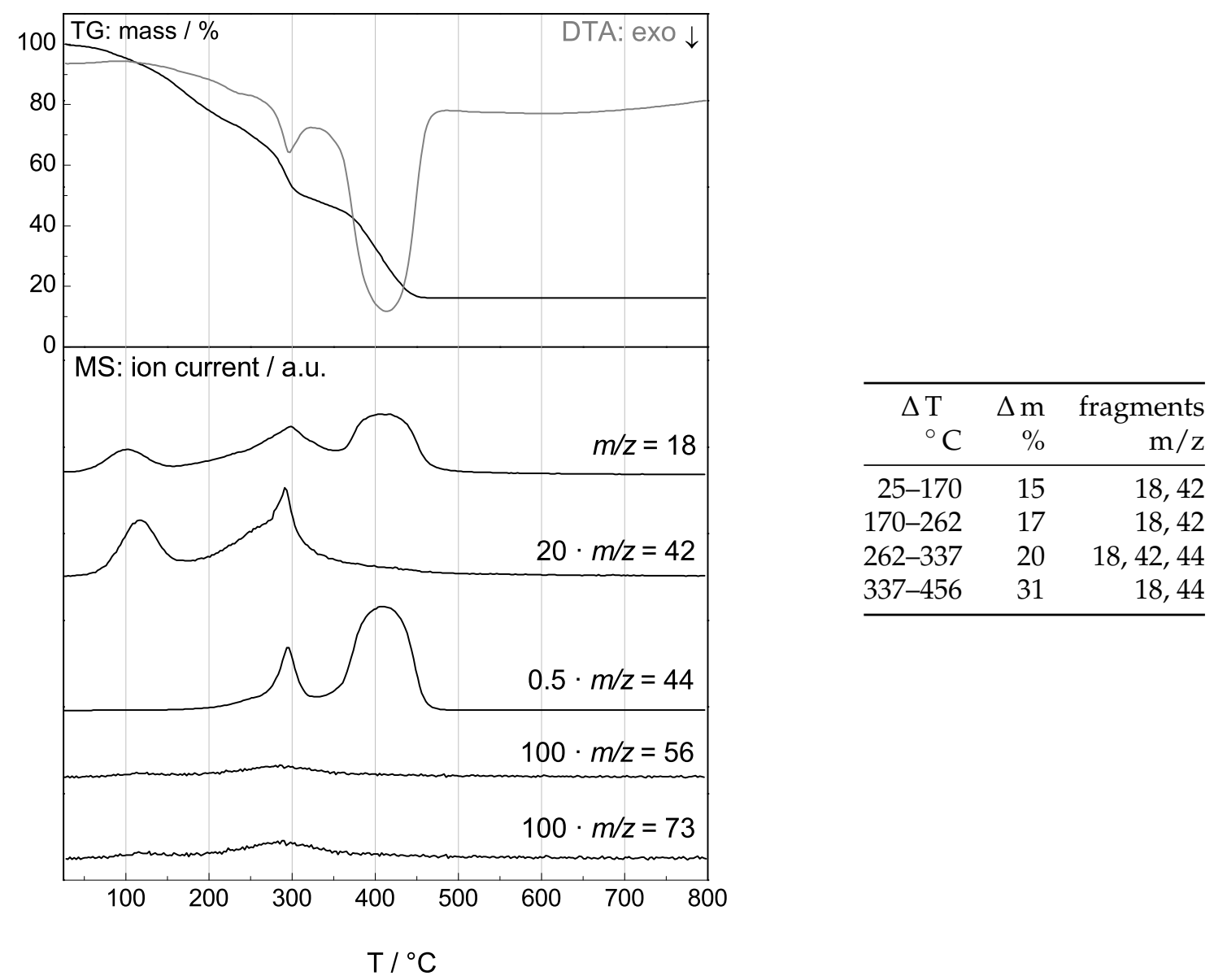

Figure S26 - TG/MS analysis of UHM-25-Val-Evans (18), weight loss (black) and DTA (grey) are plotted in the upper part and in the lower part of the diagram the characteristic single ion currents are shown for $\frac{m}{z}=18\left(\mathrm{H}_{2} \mathrm{O}\right), 42(\mathrm{THF}), 44\left(\mathrm{CO}_{2}\right), 56$ (isobutene), and $73(\mathrm{DMF})$.

\section{Catalysis and Post-Synthetic Modification}

\section{1 (3S)-1,1-Dimethoxy-3-hydroxy-butane (10)}<smiles>COC(=O)CC(C)O</smiles>

The reaction was performed in oven-dried glassware under argon. The solvent was degassed by three freeze-pump-thaw cycles prior to use. UHM-25-Pro (17) was transferred to a Schlenk tube, dried in stream of argon and then weighed in the closed reaction vessel $(220 \mathrm{mg})$. The MOF was suspended in $\mathrm{CH}_{2} \mathrm{Cl}_{2}(5 \mathrm{~mL})$ and freshly distilled acetaldehyde $(0.50 \mathrm{~mL}, 63.5 \mathrm{mg}, 1.44 \mathrm{mmol})$ were added. After five days, the reaction mixture was filtered and the remaining solid was washed with $\mathrm{CH}_{2} \mathrm{Cl}_{2}(2 \mathrm{~mL})$. The filtrate was collected in a flask containing $\mathrm{MeOH}(10 \mathrm{~mL})$ and Amberlyst $15(200 \mathrm{mg})$. After 24 hours, the reaction mixture was filtered over Celite and the filtrate was concentrated in vacuum. A clear liquid with a characteristic odor was obtained.

The reaction product was analyzed by GC-MS. The measurement was performed on a $50 \mathrm{~m}$ stationary fused silica phase modified with heptakis-(2,3-di-O-methyl-6-O-tert-butyldimethylsilyl)- $\beta$-cyclodextrin with hydrogen as the carrier gas. A complete separation of the enantiomers of the reaction product on the column was achieved using a temperature gradient of $1 \mathrm{~K} / \mathrm{min}$ after keeping the column heated to $70^{\circ} \mathrm{C}$ for five minutes after injection of the sample. The total ion chromatogram of the separation of the reaction product is shown in Figure S27. The reaction product was identified by comparison 
of the mass spectrum (see Figure S28) with reference spectra of the mass spectral database from the National Institute of Standards and Technology ${ }^{6}$

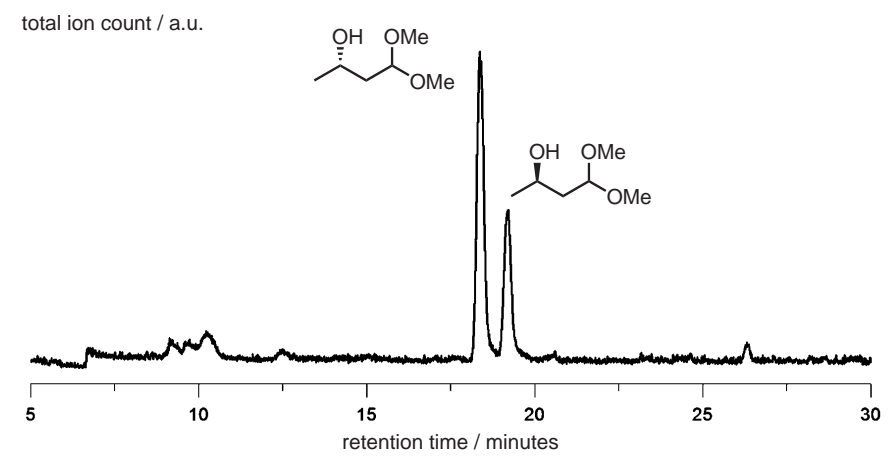

Figure S27 - Total ion chromatogram of the enantioselective gas chromatographic separation of the 1,1-dimethoxy-3-hydroxy-butane (10).

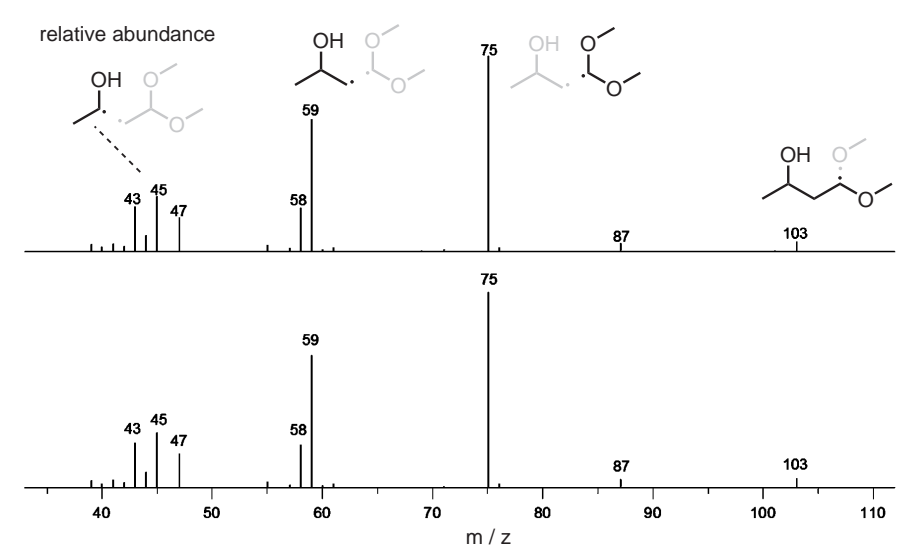

Figure S28 - Mass spectra for the peaks at retention time 18.4 (top) and 19.2 minutes (bottom) that correspond to the $(R)$ - and the $(S)$-enantiomers of 1,1-dimethoxy-3-hydroxybutane (10).

\subsection{UHM-25-Val-Evans-PSM (20)}

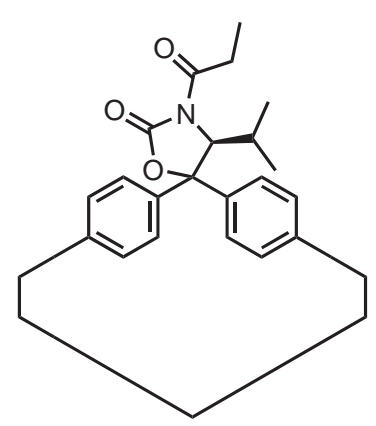

The reaction was performed in oven-dried glassware under argon. UHM-25-Val-Evans (18) was transferred to a schlenk tube, dried in stream of argon and then weighed in the closed reaction vessel $(90 \mathrm{mg}$, $0.09 \mathrm{mmol})$. The MOF was suspended in THF $(0.9 \mathrm{~mL}, 130$ eq.) and triethylamine $(0.1 \mathrm{~mL}, 13 \mathrm{eq}$.$) , a so-$ lution of 4-(dimethylamino)pyridine (DMAP) in THF $\left(183 \frac{\mathrm{mmol}}{\mathrm{L}}, 0.16 \mathrm{~mL}, 0.03 \mathrm{mmol}, 0.25 \mathrm{eq}\right.$.) and propionic anhydride $(0.16 \mathrm{~mL}, 1.2 \mathrm{mmol}, 13 \mathrm{eq}$.$) . After 72$ hours, the reaction mixture was filtered and the MOF was washed with THF $(10 \mathrm{~mL})$ and dried in vacuum. Prior to powder diffraction measurements, DMF was restored as the pore-filling solvent by solvent exchange. The powder X-ray diffractogram of the MOF after PSM and solvent exchange is shown in Figure S29. For characterization purposes, the MOF was digested in hydrochloric acid (2M) under ultrasonic irradiation. This resulted in the precipi- 
tation of the linker which was washed with water and dried in vacuum. HRMS (ESI+): 666.1961 (calc. for $\left[\mathrm{C}_{37} \mathrm{H}_{32} \mathrm{NO}_{10}+\mathrm{H}\right]^{+}$: 666.1970).

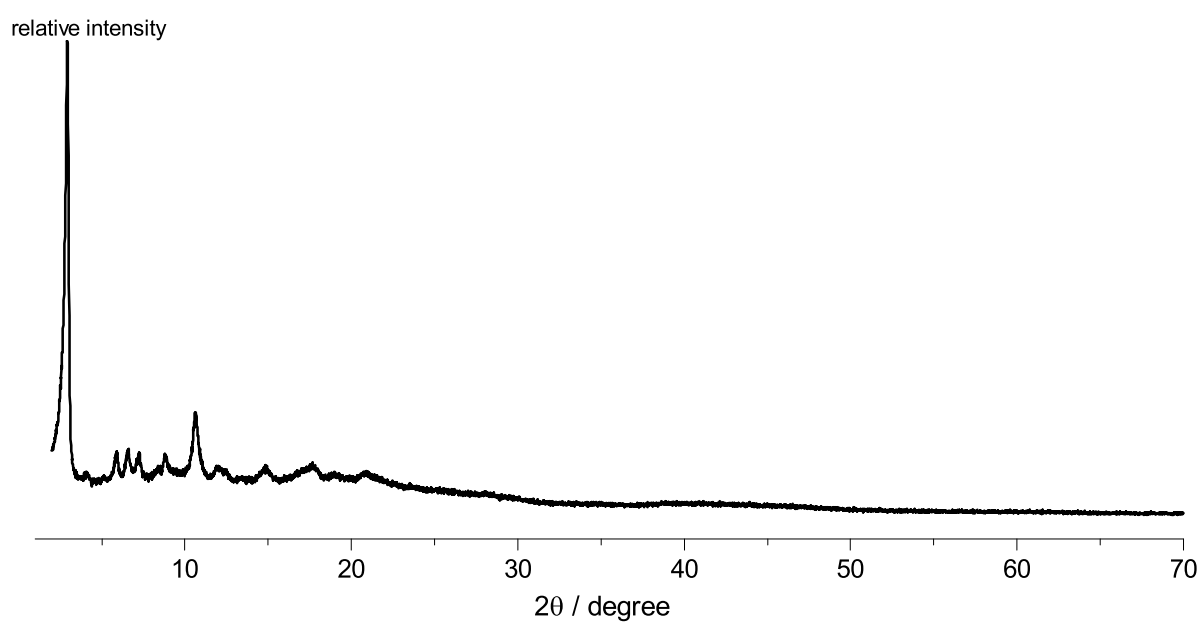

Figure S29 - Powder X-ray diffractogram of UHM-25-Val-Evans-PSM (20).

${ }^{1}$ H-NMR (300 MHz, DMSO- $\mathbf{d}_{\mathbf{6}}$ ): $\delta$ [ppm] = 14.33-12.70 (s, 4H, H20), 8.47-8.42 (m, 2H, H18), 8.40$8.33(\mathrm{~m}, 4 \mathrm{H}, \mathrm{H} 16), 7.80-7.73(\mathrm{~m}, 4 \mathrm{H}, \mathrm{H} 12, \mathrm{H} 13), 5.76-5.66(\mathrm{~m}, 1 \mathrm{H}, \mathrm{H} 5), 2.89\left(\mathrm{dq}, 1 \mathrm{H},{ }^{2} J_{\mathrm{H}-\mathrm{H}}=17.3 \mathrm{~Hz}\right.$, $\left.{ }^{3} J_{\mathrm{H}-\mathrm{H}}=7.3 \mathrm{~Hz}, \mathrm{H} 7 \mathrm{~A}\right), 2.89\left(\mathrm{dq}, 1 \mathrm{H},{ }^{2} J_{\mathrm{H}-\mathrm{H}}=17.5 \mathrm{~Hz},{ }^{3} J_{\mathrm{H}-\mathrm{H}}=7.3 \mathrm{~Hz}, \mathrm{H7B}\right), 2.22-2.04(\mathrm{~m}, 1 \mathrm{H}, \mathrm{H} 9)$, $1.01-0.88(\mathrm{~m}, 6 \mathrm{H}, \mathrm{H} 6 \mathrm{~A}, \mathrm{H} 8), 0.68\left(\mathrm{~d}, 3 \mathrm{H},{ }^{3} J_{\mathrm{H}-\mathrm{H}}=6.6 \mathrm{~Hz}, \mathrm{H} 6 \mathrm{~B}\right)$.<smiles>CCC(=O)N(C(=O)CC)C(C(C)C)C(c1ccc(-c2cc(C(=O)O)cc(C(=O)O)c2)cc1)(c1ccc(-c2cc(C(=O)O)cc(C(=O)O)c2)cc1)N(C(=O)O)C(=O)CC</smiles>

IR (KBr): $\widetilde{v}\left[\mathrm{~cm}^{-1}\right]$ = $3437(\mathrm{~m}), 2964(\mathrm{w}), 2934(\mathrm{w}), 1786(\mathrm{~s}), 1711(\mathrm{~m}), 1655(\mathrm{~s}), 1620(\mathrm{~s}), 1568(\mathrm{~s}), 1514$ (m), $1441(\mathrm{~s}), 1366(\mathrm{~s}), 1296(\mathrm{~m}), 1231(\mathrm{~m}), 1182(\mathrm{w}), 1101(\mathrm{w}), 1070(\mathrm{w}), 1020(\mathrm{~m}), 991(\mathrm{w}), 918(\mathrm{w}), 829$ $(\mathrm{w}), 812(\mathrm{w}), 772(\mathrm{~m}), 725(\mathrm{~m}), 663(\mathrm{w})$.

\section{References}

(1) Gottlieb, H. E.; Kotlyar, V.; Nudelman, A. NMR Chemical Shifts of Common Laboratory Solvent as Trace-Impurities. J. Org. Chem. 1997, 62, 7512-7515.

(2) Wuts, P. G. M.; Greene, T. W. In Greene's Protective Groups in Organic Synthesis, 4th ed.; John Wiley \& Sons, Ltd: Hoboken, NJ, USA, 2007; Chapter 7, pp 727-735.

(3) Sibi, M. P.; Zhang, R.; Manyem, S. A New Class of Modular Chiral Ligands with Fluxional Groups. J. Amer. Chem. Soc. 2003, 125, 9306-9307.

(4) Pedersen, D. S.; Rosenbohm, C. Dry Column Vacuum Chromatography. Synthesis 2001, 2001, 24312434.

(5) Spek, A. L. Structure Validation in Chemical Crystallography. Acta Crystallogr., Sect. D: Biol. Crystallogr. 2009, 65, 148-155.

(6) Wiley 9th Edition/NIST 2008 MS Library. 